\title{
ADMM Decoding of LDPC Codes: Simplification and Improvement
}

\author{
by \\ Haoyuan Wei, M.Eng.

\begin{abstract}
A Dissertation in partial fulfillment
of the requirements for the degree of
\end{abstract} \\ Doctor of Philosophy in Electrical and Computer Engineering
}

The Ottawa-Carleton Institute for Electrical and Computer Engineering

Department of Systems and Computer Engineering

Carleton University

Ottawa, Ontario

January, 2021

(C) Copyright

Haoyuan Wei, 2021 


\section{Abstract}

In this dissertation, we study linear programming (LP) decoding of low-density parity-check (LDPC) codes based on the alternating direction method of multipliers (ADMM) technique, or ADMM decoding for short. The decoding of LDPC codes is formulated as an LP model and solved efficiently by the ADMM technique. However, compared with traditional belief-propagation decoding, ADMM decoding suffers from higher complexity and worse error correction performance, which prevents the employment of ADMM decoding in reality.

To reduce the complexity of ADMM decoding, we first focus on the simplification of the check polytope projection, the most complex operation in ADMM decoding. We propose an iterative check polytope projection algorithm without the sorting operation. The proposed algorithm converges with the increase of iterations. Moreover, for a fixed number of iterations, its average complexity and the worst-case complexity are linear in the input dimension.

Another direction we propose to simplify ADMM decoding is to devise a better scheduling scheme than the standard flooding scheme. We start from the node-wise scheduling scheme which only updates the check node messages with the maximum message residual. Then we simplify the calculation of the message residual and propose a reduced-complexity node-wise scheduling scheme.

To improve the error correction performance of ADMM decoding, we propose a novel ADMM penalized decoding algorithm whose penalty term is based on check nodes $(\mathrm{CN})$. We investigate the properties of the CN penalty functions and show several examples. We make conclusions on its convergence properties and prove its failure probability is independent of the transmitted codewords for symmetric channels. Monte-Carlo simulation and the instanton analysis show its better error correction performance in the low and high SNR regions.

Finally, we propose a post-processing technique to lower the error floor of LDPC codes. The output of the first stage decoder can be revised if the syndrome is found 
in a look-up table that contains the dominant trapping sets of this code. The most complex part of the technique, the syndrome pattern matching (SPM) operation, is simplified to a one-dimensional binary search operation. Besides, a simplified SPM algorithm is proposed for quasi-cyclic (QC) LDPC codes. 
To My Families 


\section{Acknowledgments}

This is a journey with joys and tears. I encountered many amazing people. They instructed me, helped me, and accompanied me.

First of all, I would like to thank Prof. Amir H. Banihashemi for his guidance. His attitudes towards the research and devotion to one specific area are impressive. All these will have a life-long influence on me. It is also a great time working with people in his coding research group. I have a great memory of learning from their presentations.

Next, I should thank Prof. Xiaopeng Jiao and Prof. Jianjun Mu from Xidian University. They offered me suggestions both in work and life even if we cannot meet with each other as before. More importantly, their encouragement always made me full of confidence in the future.

I would like to thank my committee members. Especially, Prof. Ian Marsland kindly reviewed my draft more than once and helped improve the quality of the dissertation significantly. It is a wonderful experience to discuss with him. Prof. Yongyi Mao spared time from his busy schedule for my defense and made the procedure smooth. Prof. Frank Kschischang reviewed the dissertation so carefully, and I learned more from the insightful discussions with him.

Special thanks should be given to Prof. Wainer Gabriel, Prof. Paul Keen, and Prof. James Green. They helped me coordinate my defense and made it possible to draw a happy ending for this story.

Last but not least, I must thank my family. They have been showing their endless love and support to me during the whole period. It is so touching to feel their pride in every achievement I made, no matter how tiny it is. 


\section{Table of Contents}

Abstract $\quad$ ii

Acknowledgments $\quad$ v

Table of Contents vi vi

List of Tables $\quad$ ix

List of Figures $\quad$ x

Nomenclature $\quad$ xii

1 Introduction 1

1.1 Motivation . . . . . . . . . . . . . . . . 1

1.2 Related Works . . . . . . . . . . . . . . . . . . 2

1.2.1 Complexity Reduction of ADMM Decoding . . . . . . . . 3

1.2.2 Performance Improvement of ADMM Decoding . . . . . . . . 4

1.2.3 Error Floor Mitigation . . . . . . . . . . . . . . . 5

1.3 Summary of Contributions . . . . . . . . . . . . . . . 6

1.4 Organization of the Dissertation . . . . . . . . . . . . 8

2 Background $\quad 9$

2.1 Channel Coding . . . . . . . . . . . . . . . . . 9

2.1.1 Binary Linear Block Codes . . . . . . . . . . . . . . . 9

2.1.2 ML Decoding Model . . . . . . . . . . . . . . . . . . . 10

2.2 Binary LDPC Codes . . . . . . . . . . . . . . . . . . . . 12

2.2.1 Tanner Graphs and Trapping Sets . . . . . . . . . . . . . . . 12

2.2.2 QC-LDPC Codes ................. 14 
2.2 .3 LP Decoding Model . . . . . . . . . . . . . . . . . . . . 15

2.3 ADMM Decoding of LDPC Codes . . . . . . . . . . . . . . 17

2.3 .1 ADMM Technique . . . . . . . . . . . . 17

2.3.2 ADMM Decoding Model . . . . . . . . . . . . . . . 18

3 Iterative Projection Algorithm 22

3.1 Check Polytope and CSA-based Projection . . . . . . . . . . . 22

3.2 Iterative Polytope Projection Algorithm . . . . . . . . . . . . 25

3.3 Complexity Analysis . . . . . . . . . . . . . . . . . . . . . . . . . . . . 29

3.4 Simulation Results . . . . . . . . . . . . . . . . . . . 30

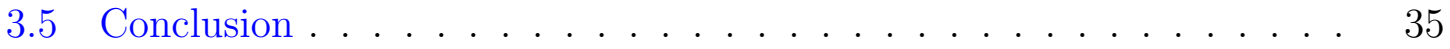

4 Reduced-Complexity Node-Wise Scheduling 36

4.1 Scheduling Schemes for BP Decoders . . . . . . . . . . . 36

4.2 Node-Wise Scheduling for ADMM Decoders . . . . . . . . . . 37

4.3 Reduced-Complexity NS-ADMM . . . . . . . . . . . . . . . 39

4.4 Computational Complexity Analysis . . . . . . . . . . . . . . 41

4.5 Simulation Results . . . . . . . . . . . . . . . . . 42

4.6 Conclusion . . . . . . . . . . . . . . . . . . . . . . 45

5 ADMM CN-Penalized Decoder $\quad 46$

5.1 ADMM VN-Penalized Decoder . . . . . . . . . . . . 46

5.2 ADMM CN-penalized Decoder . . . . . . . . . . . . . 48

5.2.1 ADMM CN-Penalized Decoding Model . . . . . . . . . . 48

5.2 .2 Possible Penalty Functions . . . . . . . . . . . . . . . . 53

5.3 Computational Complexity Analysis _. . . . . . . . . . 55

5.4 Instanton Analysis . . . . . . . . . . . . . . . . 57

5.5 Simulation Results . . . . . . . . . . . . . . . 60

5.5 .1 Selection of Parameters . . . . . . . . . . . . 60

$5.5 .2 \quad$ FER Performance . . . . . . . . . . . . . . . . 64

5.5.3 Instanton Estimation . . . . . . . . . . . . . . . 66

5.6 Conclusion . . . . . . . . . . . . . . . . . . . . . . . 70

6 Low-Complexity Post-Processing Technique $\quad 71$

6.1 Look-Up Table Based Syndrome Decoder . . . . . . . . . . . . . . 72

6.2 Low-Complexity Post-Processing Technique . . . . . . . . . 73 
6.2.1 Enhanced Look-Up Table Based Syndrome Decoder . . . . . . 73

6.2.2 SPM Algorithm for General LDPC Codes . . . . . . . . . . . 75

6.2.3 SPM Algorithm for QC-LDPC Codes . . . . . . . . . . . . . 78

6.2.4 Construction of the Look-Up Table . . . . . . . . . . . . . . . 83

6.3 Complexity Analysis . . . . . . . . . . . . . . . . . . . . . . 88

6.4 Simulation Results . . . . . . . . . . . . . . . . . . . . . . . 92

6.4.1 General LDPC code . . . . . . . . . . . . . . . . 92

6.4.2 QC-LDPC code .................... 94

6.5 Conclusion . . . . . . . . . . . . . . . . . 96

7 Future Works $\quad 97$

7.1 Check Polytope Projection Algorithm . . . . . . . . . . . . . . . . 97

7.2 Error Floor of ADMM decoding . . . . . . . . . . . . . . . . . 98

$\begin{array}{ll}\text { List of References } & 99\end{array}$

$\begin{array}{ll}\text { Appendix A Proof } & 108\end{array}$

$\begin{array}{ll}\text { Appendix B Proof } & 111\end{array}$ 


\section{List of Tables}

4.1 Complexity comparison of message updates and residual calculations per iteration for different scheduling schemes . . . . . . . . . . . . . . 42

5.1 Additional operation per iteration introduced by different penalty functions. . . . . . . . . . . . . . . . . . 56

6.1 Comparison of different feature generation functions for the random LDPC code $\mathcal{C}_{9} \ldots \ldots \ldots \ldots$. . . . . . . . . . . . . . . 86

6.2 Comparison of different feature generation function for the QC-LDPC

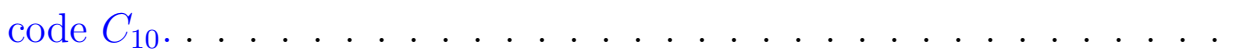

6.3 Complexity comparison of the total consumed storage and the average numbers of different operations for the proposed SPM algorithms and their variations. . . . . . . . . . . . . . . . . . 


\section{List of Figures}

2.1 A $(4,4)$ trapping set of a regular LDPC code with variable node degree 3. . . . . . . . . . . . . . . . . . . 13

2.2 The check polytope $\mathbb{P P}_{3}$ in the unit cube. . . . . . . . . . . . 16

3.1 Illustration of the iterative check polytope projection. . . . . . . . . . 27

3.2 FER performance of $\mathcal{C}_{1}$ under ADMM decoding with the proposed check polytope projection algorithm for different values of $\epsilon$ and $I_{\max }$.

3.3 Distributions of the number of projection iterations during ADMM decoding with the proposed check polytope projection algorithm for different parameters. . . . . . . . . . . . . . . . . .

3.4 Average iteration number of the proposed check polytope projection algorithm for different parameters. . . . . . . . . . . . . . . . 33

3.5 FER performance of $\mathcal{C}_{2}-\mathcal{C}_{6}$ under ADMM decoding with the proposed check polytope projection algorithm for different values of $I_{\max }$. . . .

3.6 MSE of the projection result for the proposed check polytope projection algorithm and the bisection projection algorithm for different values of

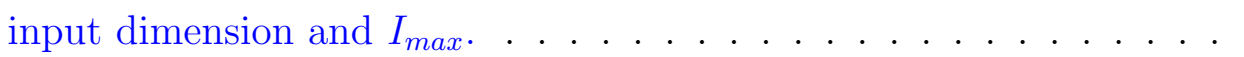

4.1 Empirical results for the estimated values of $\operatorname{Pr}(\Psi)$ for four LDPC codes at different $E_{b} / N_{0}$ values over the AWGN channel. . . . . . . .

4.2 FER performance of $\mathcal{C}_{1}$ vs. number of iterations under ADMM decoders with various scheduling schemes at $E_{b} / N_{0}=2.8 \mathrm{~dB} . . .$. . .

4.3 FER performance of $\mathcal{C}_{1}$ vs. $E_{b} / N_{0}$ under ADMM decoders with various scheduling schemes. The maximum number of iterations is set to 20 . .

4.4 Average number of iterations of $\mathcal{C}_{1}$ under ADMM decoders with various scheduling schemes at different $E_{b} / N_{0} \ldots \ldots \ldots$. . . . . . .

5.1 FER performance of $\mathcal{C}_{1}$ under the ADMM-CNP-g1 decoder plotted as a function of parameter $\mu$, the Lagrangian parameter. . . . . . . . . . 
5.2 Average number of iterations of $\mathcal{C}_{1}$ under the ADMM-CNP-g1 decoder plotted as a function of parameter $\mu$, the Lagrangian parameter. . . . 61

5.3 FER performance of $\mathcal{C}_{1}$ under the ADMM-CNP-g1 decoder plotted as a function of $\alpha$, the penalty parameter. . . . . . . . . .

5.4 FER performance of $\mathcal{C}_{1}$ under the ADMM-CNP-g1 decoder plotted as a function of $I_{\max }$, the maximum number of iterations. . . . . . .

5.5 FER performance of $\mathcal{C}_{1}$ under the proposed ADMM CN-penalized decoders and other decoders. . . . . . . . . . . . . . . 64

5.6 FER performance of $\mathcal{C}_{7}$ code under the proposed ADMM CN-penalized decoders and other decoders. . . . . . . . . . . . . 65

5.7 The minimum instanton norm of $\mathcal{C}_{8}$ under the ADMM-CNP-g1 decoder

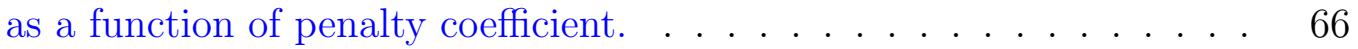

5.8 FER performance estimations of $\mathcal{C}_{8}$ under the proposed ADMM-CNPg1 decoder and other decoders. The SNR value used here is on a linear scale. . . . . . . . . . . . . . . . . .

5.9 The minimum instanton norm of $\mathcal{C}_{7}$ under the ADMM-CNP-g1 decoder as a function of penalty coefficient $\alpha . \ldots \ldots \ldots \ldots$

5.10 FER performance estimations of $\mathcal{C}_{7}$ under the proposed ADMM-CNPg1 decoder and other decoders. The SNR value used here is on a linear scale. . . . . . . . . . . . . . . . . .

6.1 Illustration of the relationship between the isomorphism class and the isomorphism sub-class. . . . . . . . . . . . . . . . .

6.2 FER performances of $\mathcal{C}_{9}$ under the quantized MS decoder and the proposed look-up table based syndrome decoder with different look-up tables. . . . . . . . . . . . . . . . . . . .

6.3 FER performances of $\mathcal{C}_{10}$ under the quantized MS decoder and the proposed look-up table based syndrome decoder with different look-up tables. . . . . . . . . . . . . . . . . . . . 


\section{Nomenclature}

\begin{tabular}{|c|c|}
\hline Acronym & Description \\
\hline AWGN & additive white gaussian noise \\
\hline ADMM & alternating direction method of multipliers \\
\hline $\mathrm{BP}$ & belief propagation \\
\hline BPSK & binary phase-shift keying \\
\hline $\mathrm{CN}$ & check node \\
\hline $\mathrm{CSA}$ & cut search algorithm \\
\hline DI & difference index \\
\hline FER & frame error rate \\
\hline LDPC & low-density parity-check \\
\hline LLR & log-likelihood ratio \\
\hline LP & linear programming \\
\hline MI & module index \\
\hline ML & maximum likelihood \\
\hline $\mathrm{MS}$ & min-sum \\
\hline $\mathrm{QC}$ & quasi-cyclic \\
\hline SNR & signal-to-noise ratio \\
\hline $\mathrm{VN}$ & variable node \\
\hline
\end{tabular}




\section{Chapter 1}

\section{Introduction}

\subsection{Motivation}

Although being forgotten for more than 30 years [1], low-density parity-check (LDPC) codes immediately ignited the researchers' passion after their rediscovery $[2,3]$. LDPC codes have advantages over traditional channel coding schemes in terms of the error correction performance. Moreover, their superior error correction performance can be achieved by the iterative message-passing decoding algorithm with low-complexity, which gives their potential of being the industry standard. Currently, they have been successfully applied in different scenarios and adopted by a number of standards, such as WiMAX [4], DVB-S2 [5], DVB-T2 [6] and IEEE 802.11n [7]. More recently, LDPC codes [8] have been selected for the 5G standard [9].

The decoding algorithm is one of the main research areas for LDPC codes. Generally, an efficient decoding algorithm should balance its complexity and error correction performance. The most widely used decoders for LDPC codes are the beliefpropagation (BP) decoder [1] and its variants [10-12]. The BP decoder has been proved to the optimal decoder when the Tanner graph [13] of the LDPC code is free of cycles [14], in the sense that it outputs the Maximum A Posteriori (MAP) estimates of the bits. However, its high complexity leads to high implementation costs. The min-sum (MS) decoders [10-12] significantly reduce the complexity of the BP decoder at the expense of slight performance degradation. The hard-decision decoders, such as the bit-flipping decoder and its variants [15-18], offer lower complexity to meet higher latency requirement but suffer more performance loss.

Although BP decoders are extensively studied and widely used, they are not the only choice for decoding LDPC codes. The decoding problem of LDPC codes can be 
formulated as a linear programming (LP) model [19] and then solved by any available LP solver. Compared with BP decoders, the LP decoding algorithm has several advantages. For example, the LP decoding algorithm has the "ML certificate", which means if the LP decoding algorithm's output is an integer vector, this output must be the maximum likelihood (ML) codeword. For BP decoders, even if the output is a valid codeword, it may not be the ML codeword. Furthermore, the performance of the LP decoding algorithm can be analyzed by the powerful tools for the optimization problem such as in [20-23]. This is attractive for ultra-reliable communication where the error correction performance should be guaranteed. The LP decoding algorithm also shows its generalization to different scenarios. It has been successfully generalized from the binary memoryless channels to non-binary LDPC codes [24,25], finite-state channels [26], and even polar codes [27].

As the LP decoding model becomes more and more popular, researchers have higher expectation on its feasibility. Several efficient algorithms were proposed to solve the LP decoding model with lower complexity [28-33]. Among them, it was proposed in [33] that the LP decoding model can be solved by the alternating direction method of multipliers (ADMM) [34] which is an efficient distributed optimization solver. The ADMM-based LP decoding algorithm, or ADMM decoding algorithm for short, enables the full utilization of LDPC codes' sparseness and therefore, dramatically reduces the complexity of LP decoding. At the same time, it keeps all the advantages of LP decoding over BP decoders. The ADMM decoding algorithm achieves a significant leap in the LP decoding area in terms of feasibility, but its complexity is still higher than BP decoders, and its error correction performance is not comparable to BP decoders, especially in the low signal-to-noise ratio (SNR) region. ${ }^{1}$ These motivate the research work on simplifying the complexity and improving the error correction performance of the ADMM decoding algorithm.

\section{$1.2 \quad$ Related Works}

Recent studies of the ADMM decoding algorithm can be mainly categorized into three parts: generalizing the application, simplifying the complexity, and improving

\footnotetext{
${ }^{1}$ For different codes, the "low SNR" regions are different. So the low SNR region in this dissertation does not refer to a specific range, but to a code-dependent SNR range where its frame error rate (FER) is less than $10^{-4}$.
} 
the error correction performance. As a successful generalization, the ADMM decoding algorithm is combined with channel detection, and turbo equalization is used to improve the system's performance in [35]. The ADMM decoding model is also applied to non-binary codes [36] and multi-permutation codes [37]. More recently, the ADMM decoding algorithm is used for partial response channels [38], and its complexity increases linearly with the channel memory length. More works focusing on the other two categories will be explained in detail as follows. They are also our study directions. [19]

\subsubsection{Complexity Reduction of ADMM Decoding}

To reduce the complexity of ADMM decoding, most works focus on simplifying the check polytope projection operation, the most time-consuming and complicated component in ADMM decoding. The original check polytope projection algorithm of [33] involves two sorting operations. Zhang and Siegel [39] proposed a projection algorithm based on the cut-search algorithm of [40]. This algorithm requires only one sorting operation and greatly decreases the computation time. Both projection algorithms of [33] and [39] have an average complexity $O(d \log d)$, where $d$ is the input dimension, because of the use of the sorting operation ${ }^{2}$. The projection algorithm was later simplified in [41] by transforming the projection onto the check polytope to the projection onto a simplex. This algorithm involves a partial sorting operation other than the standard sorting operation, which has an average complexity $O(d)$ and a worst-case complexity $O\left(d^{2}\right)$. In [42], the authors focused on the hardware implementation of the projection algorithm. They combined the ideas of the cutsearch algorithm and simplex projection and adapted them for the implementation in a field-programmable gate array (FPGA). The projection algorithm has the same computational complexity as [41] since it also uses only the partial sorting operation. However, as pointed out in [42], there is no efficient hardware implementation for the partial sorting currently. As a result, the partial sorting operation still has to be implemented as the standard sorting operation, and the advantage in terms of the complexity brought by the partial sorting operation is lost. In [43], Wei et al. reduced the computational complexity of ADMM decoding by reducing the number of polytope projections instead of reducing the complexity of the projection itself. The unnecessary projection operations are avoided during the iteration, leading to a

\footnotetext{
${ }^{2}$ In this dissertation, we assume the sequential quicksort is used.
} 
significant reduction of the execution time. Jiao et al. used look-up tables to replace the complexity projection operation at the expense of large memory and developed efficient look-up table searching algorithms [44,45].

Based on the iterative projection algorithm in [46], the bisection search was proposed in [47] to accelerate the convergence of the algorithm. The algorithm in [46] was also accelerated in [48] by using a closed-form approximation when the iterative procedure is close to the end. In [49], the input point is projected onto the line segment defined by the two nearest points to the input point in the check polytope. Another iterative projection algorithm was proposed in [50], and the result of the projection is determined element by element in each iteration.

There are also works simplifying ADMM decoding from other aspects. For example, layered scheduling was successfully applied to ADMM decoding [51], and it achieved a faster convergence speed than the flooding scheme in [33]. We note that it is also possible to apply other scheduling schemes [52-61] proposed for BP decoders on ADMM decoding. As a trade-off between decoding time and memory consumption, the ADMM technique is applied to the LP decomposition model [29] which is equivalent to the original LP decoding model, but with more auxiliary variables, in $[62,63]$. Other successful simplifications include a software implementation of the ADMM decoding algorithm on a multi-core platform in [64] and a hardware implementation of the ADMM decoding algorithm [65].

\subsubsection{Performance Improvement of ADMM Decoding}

To improve the error correction performance of ADMM decoding at low SNR values, penalty terms were added to the LP model's objective function to penalize the pseudo-codeword. Pseudo-codewords are the non-integer points in the feasible region of ADMM decoding but have smaller objective function values than codewords. Since ADMM decoding outputs the solution which has the smallest objective function value from its feasible region, the penalty terms add extra barriers for the pseudocodewords to be output. Several simple and efficient functions that can be used as penalty terms were given in [66]. For irregular codes, Jiao et al. proposed the idea of assigning different penalty coefficients to variable nodes with different degrees in [67]. They efficiently optimized the multiple penalty coefficients by differential evolution,

and the frame error rate (FER) performance of the decoder is further improved. In [68], Wang et al. used a segmented function as the penalty term and optimized 
the relevant parameters by differential evolution. The convergence speed and the FER performance are shown to be improved. These decoders all penalize the invalid solutions from the aspect of variable nodes.

More recently, deep learning is used to aid the performance improvement of ADMM decoding in [69]. A neural network is constructed by unfolding the ADMM iterations. As a result, the parameter values of ADMM decoding can be different in different iterations. Thus the parameters in each iteration of ADMM decoding can be learned simultaneously. These fully optimized parameters provide an extra performance gain for ADMM decoding. In [70], the $\ell_{p}$ box constraints approximating the binary constraints are added to the ADMM decoding model. The newly introduced constraints remove some pseudo-codewords in the feasible region and significantly improve the FER performance of ADMM decoding at the expense of higher complexity.

\subsubsection{Error Floor Mitigation}

The FER of LDPC codes usually drops sharply as the noise level decreases in the lowto-medium SNR region (also known as the waterfall region). When the Tanner graph of the LDPC code has cycles, the FER reduction slows down as the noise level keeps decreasing in the high SNR region. This is the notorious error floor phenomenon and can be explained by trapping sets [71,72]. Plenty of effort has been made to mitigate the error floor phenomenon from the aspect of trapping sets. Some of them tried to construct good codes without harmful trapping sets [72-81]. The others try to devise better decoding algorithms which can break the trapping sets. Most of the decoding algorithms are based on the information provided by unsatisfied check nodes (syndrome pattern). Cavus et al. stored dominant trapping sets in a look-up table and reversed the erroneous decoding result if one matched syndrome pattern is found in the look-up table [82]. Han and Ryan analyzed some specific codes and proposed a few low-floor decoders which erased some bits affected by the trapping sets after a decoding failure [83]. A post-processing approach was formulated to exploit the structure of the absorbing set by increasing the reliability of the message from the unsatisfied checks and reducing the reliability of the messages from satisfied checks in [84]. Kang et al. proposed a trial-and-error technique that tried to flip one bit neighboring to unsatisfied check nodes each time until re-decoding is successful [85]. Specifically for ADMM decoding, the dominant trapping sets are studied in [86], and the code modification is proposed to eliminate the harmful trapping sets of LDPC 
codes.

\subsection{Summary of Contributions}

In this dissertation, we propose to address the challenges as described in the following. Our main contributions in this dissertation, which are published or submitted for publication in [46], [87], [88], [89], and [90] can be summarized as follows:

- In [46], we propose an iterative check polytope projection algorithm for ADMM decoding. We prove that the projection algorithm converges with the increase of the iteration.

- In [87], we apply node-wise scheduling to ADMM decoding and also devise a reduced-complexity node-wise scheduling scheme.

- In [88], we present a revised proof of the all-zero assumption for the ADMM penalized decoding algorithms in [66].

- In [89], we propose a novel ADMM check node penalized decoding algorithm. We investigate the convergence properties of the proposed algorithm and prove that its failure probability is independent of the transmitted codewords for symmetric channels.

- In [90], we design a look-up table based post-processing technique to lower the error floor of LDPC codes. A binary search operation is used to simplify the array matching operation. For quasi-cyclic (QC) LDPC codes, a further simplified array matching algorithm is proposed.

As we noted before, the check polytope projection is the most time-consuming operation in ADMM decoding. All previous projection algorithms require sorting or partial sorting operations. The existence of the sorting operation introduces an average complexity of $O(d \log (d))$ and a worst-case complexity of $O\left(d^{2}\right)$. More importantly, the sorting or the partial sorting operation consumes much more hardware resources than operations of MS decoders, which prevents the industrial deployment of the ADMM decoder. We propose an iterative check polytope projection algorithm that does not require sorting. The operations involved are simple arithmetic operations, and the projection result is refined in each iteration. For a fixed number of 
iterations, the computational complexity of the proposed algorithm is linear in $d$. The convergence of the proposed algorithm is proved. Simulation results show that the proposed projection algorithm can achieve a similar FER performance with the accurate projection algorithm.

The ADMM decoding algorithm can be seen as an iterative message-passing algorithm. For a message-passing algorithm, the scheduling of messages plays an important role in the convergence of the algorithm. The standard flooding scheme updates all check node messages or all variable node messages in one iteration, which involves a significant number of unimportant updating operations. Inspired by the effective scheduling schemes developed for BP decoders, we investigate the node-wise scheduling scheme which only updates the check node messages with the maximum message residual in each iteration. Unlike BP decoders, the calculation of the check node message residuals involves the polytope projection operation which consumes non-negligible time. Thus, we simplify the calculation of the message residual and propose a reduced-complexity node-wise scheduling scheme. Simulation results show that the simplified scheduling scheme significantly accelerates the convergence speed of ADMM decoding compared with the flooding scheme and the layered scheduling.

By adding penalty terms to the objective function of the LP decoding model, the FER performance of ADMM decoding can be improved in the low SNR region. All previous ADMM penalized decoding algorithms can be categorized as ADMM variable node (VN) penalized decoding algorithms since they penalize the non-integer values of variable nodes. In contrast, we propose a novel ADMM penalized decoding algorithm whose penalty terms are focused on check nodes $(\mathrm{CN})$. Solutions that cannot satisfy all parity-checks will have a larger value of penalty terms than the valid codewords. We term it the ADMM CN-penalized decoding algorithm. Description of functions that can be used as CN-penalty terms is present, and several examples are given. The convergence conclusion and the all-zero assumption of the ADMM CNpenalized decoding algorithm are proved. The complexity of the proposed algorithm with different penalty functions is also analyzed. Simulation results show that the proposed algorithm has a better or similar performance than ADMM VN-penalized decoding algorithms in the simulated range. The instanton analysis method [91] is used to estimate the performance of the proposed algorithm at high SNR values. It shows that the ADMM CN-penalized decoding algorithm will continue outperforming the ADMM VN-penalized decoding algorithms. We also provide a revised proof of 
the all-zero assumption for the ADMM VN-penalized decoding algorithms.

Finally, we propose a look-up table based post-processing technique to lower the error floor of LDPC codes. When the first stage decoder of an LDPC code fails to output a codeword, the syndrome pattern will be searched in a look-up table that contains the dominant trapping sets. The output of the first stage decoder can be directly flipped based on the information of the matched trapping set. The most complex part of the technique, the syndrome pattern matching (SPM) operation ${ }^{3}$, is simplified to a one-dimensional binary search operation by extracting a feature value from each trapping set. A further simplified SPM algorithm is proposed for QC LDPC codes. We analyze both the computation complexity and the storage complexity of the proposed method. The simulation results show the trade-off between performance improvement and complexity. It should be noted that the proposed post-processing technique can be potentially combined with the ADMM decoding algorithm.

\subsection{Organization of the Dissertation}

The rest of the dissertation is organized as follows. Basic definitions, notations and backgrounds are provided in Chapter 2. In Chapter 3, we investigate the check polytope projection operation. In Chapter 4, we study the scheduling strategy of the ADMM decoding algorithm. In Chapter 5, the ADMM CN-penalized decoding algorithm is presented. In Chapter 6, we propose a low-complexity post-processing technique that could be used in ADMM decoding. Some ideas for future work are presented in Chapter 7.

\footnotetext{
${ }^{3}$ The syndrome pattern is an array of integer indices, and the syndrome pattern matching operation is to find whether the array exists in a list of arrays
} 


\section{Chapter 2}

\section{Background}

A communication system aims to transmit messages efficiently and reliably. It has been shown by Claude Shannon as early as 1948 [92] that channel coding is an inevitable part of the communication system to combat noise disturbance. As a class of channel coding schemes, LDPC codes have received tremendous attention because of their excellent error correction performance.

In this chapter, we first give the background of channel coding in Section 2.1. Then the basic properties of LDPC codes are explored and its LP decoding model is presented in Section 2.2. Finally, the ADMM technique is explained and used to solve the LP decoding model in Section 2.3.

\subsection{Channel Coding}

Binary linear block codes are a family of channel coding schemes [93]. They are used throughout this dissertation. In this section, the concept of binary linear block codes is introduced, and the ML decoding model of binary linear block codes is explained.

\subsubsection{Binary Linear Block Codes}

For an $(n, k)$ binary linear block code, a sequence of information bits are divided into groups of $k$ bits. For each group, an encoder generates and adds $n-k$ redundant bits based on some rules. The final $n$ bits, called a codeword, are transmitted via a channel. The code-rate is then defined as $k / n$. The channel output may not be exactly the same as the transmitted codeword due to the existence of the channel noise. A decoder will then be used to recover the transmitted codeword with or 
without knowing the channel property.

The encoding process can be done with the assistance of a generator matrix $\mathbf{G}$. For an $(n, k)$ binary linear block code, $\mathbf{G}$ is of size $n \times k$ and rank $k$. For a transmitted information sequence $\mathbf{m}$ of size $k \times 1$, the transmitted codeword $\mathbf{x}$ is obtained by the following modulo-2 operation:

$$
\mathrm{x}=\mathrm{Gm} .
$$

All possible transmitted codewords form a codeword set

$$
\mathcal{C}=\left\{\mathbf{G m} \mid \mathbf{m} \in\{0,1\}^{k}\right\} .
$$

The decoding process can be done with the assistance of a parity check matrix $\mathbf{H}$. For an $(n, k)$ binary linear block code, $\mathbf{H}$ is of size $m \times n$ and rank $n-k$, where $m \geq n-k$. For any codeword $\mathbf{x} \in \mathcal{C}$, the following module-2 equality always holds

$$
\mathrm{Hx}=\mathbf{0} .
$$

Therefore, the parity check matrix can be used to judge if a binary sequence is a valid codeword. Each row of (2.3) is one parity-check equation. This procedure is called parity checking, which can only be performed on a binary sequence. For a fractional sequence, the parity checking is performed on its hard-decision sequence, which is obtained bit-wisely by

$$
\hat{x}_{i}= \begin{cases}1, & \text { if } x_{i}>0.5 \\ 0, & \text { if } x_{i}<0.5\end{cases}
$$

\subsubsection{Decoding Model}

Suppose the transmitted codeword is $\mathbf{x} \in \mathcal{C}$ and the received sequence is $\mathbf{y}$. A decoder is to find one estimated codeword $\hat{\mathbf{x}}$ which is the most-likely transmitted codeword on the condition that the received sequence is $\mathbf{y}$. This principle can be described by the following model:

$$
\hat{\mathbf{x}}=\arg \max _{\mathbf{x} \in \mathcal{C}} \operatorname{Pr}(\mathbf{x} \mid \mathbf{y}) .
$$

This model estimates the transmitted codeword based on the received sequence, which is called maximum-a-posteriori (MAP) decoding. Based on Bayes' rule, and the 
assumption that codewords in the codeword set will be sent with equal probability, the MAP decoding is equivalent to the following ML decoding model:

$$
\hat{\mathbf{x}}=\arg \max _{\mathbf{x} \in \mathcal{C}} \operatorname{Pr}(\mathbf{y} \mid \mathbf{x}) .
$$

In a binary memoryless channel, all bits are affected by the noise independently, yielding that $\hat{\mathbf{x}}=\arg \max _{\mathbf{x} \in \mathcal{C}} \prod_{1}^{n} \operatorname{Pr}\left(y_{i} \mid x_{i}\right)$. By taking the logarithm of the right-hand side, we have $\hat{\mathbf{x}}=\arg \max _{\mathbf{x} \in \mathcal{C}} \sum_{1}^{n} \log \operatorname{Pr}\left(y_{i} \mid x_{i}\right)=\arg \min _{\mathbf{x} \in \mathcal{C}}-\sum_{1}^{n} \log \operatorname{Pr}\left(y_{i} \mid x_{i}\right)$.

After adding the constant $\sum_{1}^{n} \log \operatorname{Pr}\left(y_{i} \mid 0\right)$ to the right-hand side, we have

$$
\begin{aligned}
\hat{\mathbf{x}} & =\min _{\mathbf{x} \in \mathcal{C}} \sum_{i=1}^{n}\left(\log \operatorname{Pr}\left(y_{i} \mid 0\right)-\log \operatorname{Pr}\left(y_{i} \mid x_{i}\right)\right) \\
& =\min _{\mathbf{x} \in \mathcal{C}} \sum_{i=1}^{n} \log \frac{\operatorname{Pr}\left(y_{i} \mid 0\right)}{\operatorname{Pr}\left(y_{i} \mid x_{i}\right)} \\
& =\min _{\mathbf{x} \in \mathcal{C}} \sum_{i: x_{i}=0} \log \frac{\operatorname{Pr}\left(y_{i} \mid 0\right)}{\operatorname{Pr}\left(y_{i} \mid 0\right)}+\sum_{i: x_{i}=1} \log \frac{\operatorname{Pr}\left(y_{i} \mid 0\right)}{\operatorname{Pr}\left(y_{i} \mid 1\right)} \\
& =\min _{\mathbf{x} \in \mathcal{C}} \sum_{i=1}^{n} \log \frac{\operatorname{Pr}\left(y_{i} \mid 0\right)}{\operatorname{Pr}\left(y_{i} \mid 1\right)} \cdot x_{i} \\
& =\min _{\mathbf{x} \in \mathcal{C}} \sum_{i=1}^{n} \gamma_{i} \cdot x_{i},
\end{aligned}
$$

where $\gamma_{i}=\log \frac{\operatorname{Pr}\left(y_{i} \mid 0\right)}{\operatorname{Pr}\left(y_{i} \mid 1\right)}$ is called log-likelihood ratio (LLR). It means the ML decoding problem tries to find a codeword in the codeword set $\mathcal{C}$ which has the minimum inner product value with LLR vector. Considering the condition (2.3) that implies $\mathbf{x} \in \mathcal{C}$, the ML decoding model can be described in the following model:

$$
\begin{aligned}
\operatorname{minimize} & \boldsymbol{\gamma}^{T} \mathbf{x} \\
\text { subject to } & \mathbf{H x}=0, \\
& x_{i} \in\{0,1\}, \quad i=1,2, \ldots, n .
\end{aligned}
$$

The additive white gaussian noise (AWGN) channel is the channel model used in this dissertation. Its input is discrete, and its output is continuous, namely, $y_{i}=$ $x_{i}+n_{i}$, where $x_{i}$ is the discrete channel input, and $n_{i}$ is a normally distributed random variable with mean 0 and standard deviation $\sigma$. The probability density function of 
$y_{i}$ is

$$
y_{i}=\frac{1}{\sqrt{2 \pi \sigma^{2}}} \exp \left(-\frac{\left(y_{i}-x_{i}\right)^{2}}{2 \sigma^{2}}\right) .
$$

For the AWGN channel with binary phase-shift keying (BPSK), the component of the LLR vector is

$$
\begin{aligned}
\gamma_{i} & =\log \frac{\operatorname{Pr}\left(y_{i} \mid x_{i}=0\right)}{\operatorname{Pr}\left(y_{i} \mid x_{i}=1\right)}=\log \frac{\operatorname{Pr}\left(y_{i} \mid+1\right)}{\operatorname{Pr}\left(y_{i} \mid-1\right)} \\
& =\log \frac{\frac{1}{\sqrt{2 \pi \sigma^{2}}} \exp \left(-\frac{\left(y_{i}-1\right)^{2}}{2 \sigma^{2}}\right)}{\frac{1}{\sqrt{2 \pi \sigma^{2}}} \exp \left(-\frac{\left(y_{i}+1\right)^{2}}{2 \sigma^{2}}\right)} \\
& =\frac{\left(y_{i}+1\right)^{2}-\left(y_{i}-1\right)^{2}}{2 \sigma^{2}} \\
& =\frac{2 y_{i}}{\sigma^{2}} .
\end{aligned}
$$

\subsection{Binary LDPC Codes}

Binary LDPC codes are a class of binary linear codes. So they can also be represented by the generator matrix or the parity check matrix. What makes LDPC codes special is that their parity check matrix is sparse. It means the number of non-zero entries in one row of the parity check matrix is small compared with the total length of the row.

In this section, the Tanner graph representation of the LDPC code is explained, and the concept of trapping sets is introduced. The efficient representation of trapping sets of the QC-LDPC code is given. Finally, the LP decoding model is explained.

\subsubsection{Tanner Graphs and Trapping Sets}

LDPC codes can be represented by bipartite graphs called Tanner graphs [13]. The graphs include two kinds of nodes, variable nodes and check nodes. For an $(n, k)$ binary LDPC code, $n$ variable nodes indicate the codeword bits, and $m=n-k$ check nodes indicate the parity-check equations. An edge $\left(v_{i}, c_{j}\right)$ connects variable node $v_{i}$ and check node $c_{j}$ if and only if $H_{j i}=1$. Let $\mathcal{I}=\{1,2, \ldots, n\}$ and $\mathcal{J}=$ $\{1,2, \ldots, m\}$ be the index sets of variable nodes and check nodes, respectively. The 


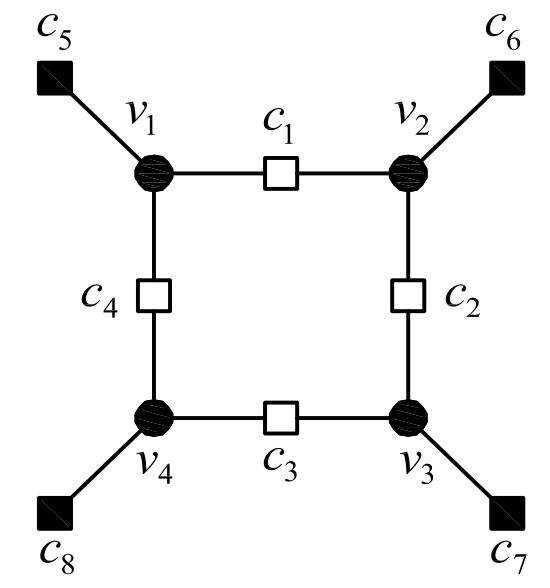

Figure 2.1: A $(4,4)$ trapping set of a regular LDPC code with variable node degree 3. Circles represent variable nodes, and squares represent check nodes. The four solid circles represent four variable nodes in an erroneous state, and the four solid squares represent four unsatisfied check nodes. The four empty squares represent four mis-satisfied check nodes.

set of neighboring check nodes of variable node $i$ is denoted by $N_{v}(i)$. The degree of variable node $i$, denoted by $d_{i}$, is the cardinality of $N_{v}(i)$. Similarly, the set of neighboring variable nodes of check node $j$ is denoted by $N_{c}(j)$. The degree of check node $j$ is denoted by $d_{j}$ with a slight abuse of notation.

An $(a, b)$ trapping set is a set $T$ of $a$ message bits in error that result in $b$ unsatisfied parity-checks. The indices of these $a$ message bits form a vector, called the error pattern, and the index vector of the $b$ unsatisfied checks is called the syndrome pattern. These definitions are slightly different from other references where the error pattern and the syndrome pattern may refer to the sparse binary vectors of length $n$ and $m$, respectively. Here we only record the locations of their non-zero components but still call them the error pattern or the syndrome pattern, respectively. A trapping set can be represented by a sub-graph of the code's Tanner graph. Fig. 2.1 gives a $(4,4)$ trapping set with $T=\left\{v_{1}, v_{2}, v_{3}, v_{4}\right\}$. During the decoding procedure, if the variable nodes in $T$ are in erroneous states, check nodes $\left\{c_{5}, \ldots, c_{8}\right\}$ are unsatisfied, and check nodes $\left\{c_{1}, \ldots, c_{4}\right\}$ are mis-satisfied. Although there exist some unsatisfied parity-checks, these unsatisfied check nodes may not be able to correct the errors, and the decoder will be trapped.

Since avoiding or compensating for trapping sets can be used to lower the error floor, plenty of works have focused on searching for the trapping sets of LDPC codes $[82,84,94-98]$. Methods for finding the trapping sets can be divided into three 
classes. The first one is based on simulations. FPGA was used in the simulation to accelerate the searching and can obtain a sufficiently large list of dominant trapping sets in [84]. Cavus et al. used importance sampling to narrow the searching space [82]. The simulation time could be reduced significantly, and a partial list of dominant trapping sets can be obtained. The second class is based on graph theory. In [94], the original Tanner graph of the code is transformed into an augmented Tanner graph, and a weight-enumeration algorithm is applied. Karimi and Banihashemi characterized leafless elementary trapping sets as layered supersets of short cycles in the code's Tanner graph and proposed an efficient algorithm for finding dominant trapping sets [95]. More recently, Hashemi and Banihashemi presented an improved characterization of leafless elementary trapping sets. Their proposed efficient exhaustive search algorithms were proved to be able to find all leafless elementary trapping sets smaller than size $(a, b)$ [97] [98]. The third class is based on the branch-\&-bound principle. Kyung and Wang formulated the searching algorithm as an integer programming problem and solved it based on this principle [96].

\subsubsection{QC-LDPC Codes}

QC-LDPC codes are a special kind of LDPC codes that are widely deployed in the industry. Their parity-check matrices can be broken into sub-matrices that have circulant structures. The circulant structure brings an advantage to the representation of trapping sets. For a QC-LDPC code with the $Q \times Q$ circulant sub-matrix, the isomorphism class of trapping sets is of size $Q$. In other words, if one can find a single trapping set, then there exist $Q-1$ other trapping sets that can be generated by applying different quasi-cyclical shift amounts, $\Delta$, on this trapping set. Mathematically, if one $(a, b)$ trapping set is denoted by an error pattern $\left\{v_{1}, v_{2}, \ldots, v_{a}\right\}$ and a syndrome pattern $\left\{c_{1}, c_{2}, \ldots, c_{b}\right\}$, its isomorphic trapping set with QC-shift amount $\Delta$ can be obtained by

$$
\begin{aligned}
& v_{i}^{\Delta}=\left\lfloor\left(v_{i}-1\right) / Q\right\rfloor * Q+\left(\left(v_{i}-1\right) \% Q+\Delta\right) \% Q+1, \quad i=1,2, \ldots, a, \\
& c_{j}^{\Delta}=\left\lfloor\left(c_{j}-1\right) / Q\right\rfloor * Q+\left(\left(c_{j}-1\right) \% Q+\Delta\right) \% Q+1, \quad j=1,2, \ldots, b,
\end{aligned}
$$

where $\left\lfloor\left(v_{i}-1\right) / Q\right\rfloor$ is the quotient of the integer division and called the module index of variable node $v_{i} ;\left(v_{i}-1\right) \% Q$ is the remainder of the integer division and called 
the circulant index of variable node $v_{i}$ [93]; $\Delta$ is the QC-shift amount and could be $1,2, \ldots, Q-1$. Similarly, $\left\lfloor\left(c_{j}-1\right) / Q\right\rfloor$ and $\left(c_{j}-1\right) \% Q$ are called module index and circulant index of check node $c_{j}$, respectively. The indices in the dissertation all start from 1. It can be found that the module index of any variable nodes or check nodes does not change no matter which shift amount $\Delta$ is applied. $\left(\left\lfloor\left(c_{1}-1\right) / Q\right\rfloor,\left\lfloor\left(c_{2}-\right.\right.\right.$ $\left.1) / Q\rfloor, \ldots,\left\lfloor\left(c_{b}-1\right) / Q\right\rfloor\right)$ is then called module index vector of the syndrome patterns of this isomorphism class (or MI vector for short). We note that $Q$ is also called the lifting degree.

\subsubsection{LP Decoding Model}

The ML decoding model (2.8) is an integer programming (IP) model which is infeasible for large size. In [19], the authors proposed the following relaxed LP model to recover the transmitted symbols $\mathbf{x}$ in the codeword set $\mathcal{C}$ :

$$
\begin{aligned}
\text { minimize } & \gamma^{T} \mathbf{x} \\
\text { subject to } & \mathbf{P}_{j} \mathbf{x} \in \mathbb{P P}_{d_{j}}, \quad \forall j \in \mathcal{J},
\end{aligned}
$$

where $\gamma$ is the LLR vector of length $n$. $\mathcal{J}$ is the index sets of check nodes. $\mathbf{P}_{j}$ is a $d_{j} \times n$ binary matrix that contains 1 s only in the $s$-th row and the $t$-th column if $v_{t}$ is the $s$-th neighboring variable node of check node $j$. Therefore, $\mathbf{P}_{j} \mathbf{x}$ is the sub-vector of $\mathbf{x}$, and it is composed of the values of all variable nodes checked by the $j$-th check node. $\mathbb{P}_{d_{j}}$ is the set of even weight binary vectors of length $d_{j} . \mathbb{P P}_{d_{j}}$ is defined as the convex hull of set $\mathbb{P}_{d_{j}}$ and is also called the check polytope.

Example 1. Suppose the set of neighboring variable nodes of check node $j$ is $N_{c}(j)=$ $\{1,3,5,6\}$ and the code length is $n=6$. The selection matrix of check node $j$ is

$$
\mathbf{P}_{j}=\left[\begin{array}{llllll}
1 & 0 & 0 & 0 & 0 & 0 \\
0 & 0 & 1 & 0 & 0 & 0 \\
0 & 0 & 0 & 0 & 1 & 0 \\
0 & 0 & 0 & 0 & 0 & 1
\end{array}\right] .
$$




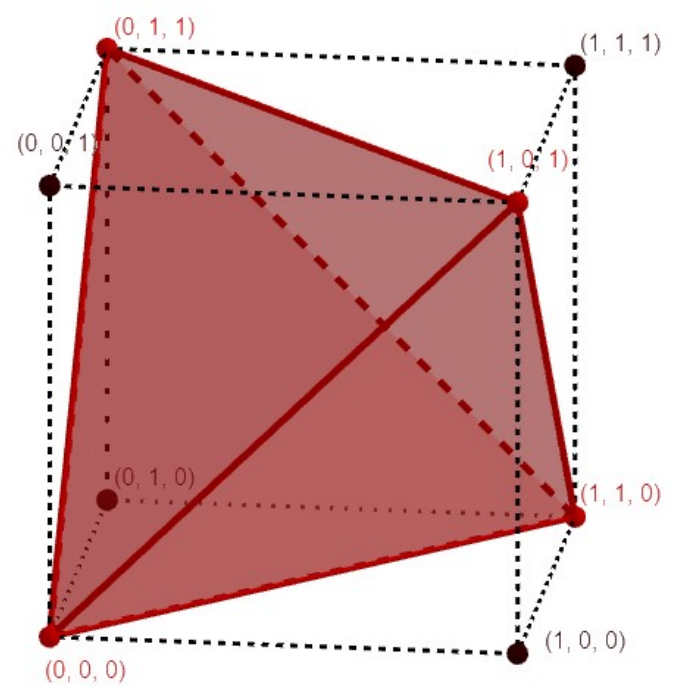

Figure 2.2: The check polytope $\mathbb{P P}_{3}$ in the unit cube. $\mathbb{P}_{3}$ is the set $\{000,110$, $101,011\} . \mathbb{P P}_{3}$ is the pyramid in maroon.

Then we have

$$
\mathbf{P}_{j} \mathbf{x}=\mathbf{P}_{j} \cdot\left[x_{1}, x_{2}, x_{3}, x_{4}, x_{5}, x_{6}\right]=\left[x_{1}, x_{3}, x_{5}, x_{6}\right]
$$

Example 2. In the case where $d_{j}=4, \mathbb{P}_{4}$ is the set $\{0000,1100,1010,1001,0110$, 0101,0011,1111\}. It contains all points of length 4 with even weight (for example, 0 , 2 , and 4$)$. Then $\mathbb{P P}_{4}$ contains all points in the convex hull of $\mathbb{P}_{4}$.

In the case where $d_{j}=3, \mathbb{P}_{3}$ is the set $\{000,110,101,011\}$. Then $\mathbb{P P}_{3}$ contains all points in the convex hull of $\mathbb{P}_{3}$, which is the pyramid shown in Fig. 2.2.

The condition $\mathbf{P}_{j} \mathbf{x} \in \mathbb{P}_{d_{j}}$ indicates that the sub-vector of $\mathbf{x}$ checked by the $j$ th check node is composed of an even number of $1 \mathrm{~s}$. This implies the parity-check equation related to the $j$-th check node will be satisfied. If for all $j \in \mathcal{J}$, the condition $\mathbf{P}_{j} \mathbf{x} \in \mathbb{P}_{d_{j}}$ holds, it means $\mathbf{x}$ is a valid codeword, namely $\mathbf{x} \in \mathcal{C}$. As a result, if the constraint $\mathbf{P}_{j} \mathbf{x} \in \mathbb{P P}_{d_{j}}$ in (2.13) is replaced by $\mathbf{P}_{j} \mathbf{x} \in \mathbb{P}_{d_{j}}$, the model will become an IP model equivalent to (2.8), and the solution of the IP model must be the ML solution. In contrast, the solution of the LP model (2.13) will be the ML solution only if the output is an integer solution. This property is called "ML Certificate". An operator $\prod_{\mathbb{P}_{d_{j}}}(\cdot)$ is defined as the Euclidean projection onto check polytope $\mathbb{P P}_{d_{j}}$, which means finding the nearest point in the polytope $\mathbb{P P}_{d_{j}}$ to the given input point. 


\subsection{ADMM Decoding of LDPC Codes}

In this section, the ADMM technique is reviewed, and ADMM decoding of LDPC codes is explained.

\subsubsection{ADMM Technique}

The ADMM technique is a distributed method for solving general convex optimization problems based on the idea of decomposition and coordination [34]. The large optimization problem is decomposed into small-sized problems and solved locally. Then the local minimums are coordinated into the global minimum. The ADMM technique can be regarded as the method of combining the advantages of dual decomposition and augmented Lagrangian.

Specifically, the ADMM technique can be used to solve the following optimization problem:

$$
\begin{aligned}
\operatorname{minimize} & f(\mathbf{x})+g(\mathbf{z}) \\
\text { subject to } & \mathbf{A x}+\mathbf{B z}=\mathbf{c},
\end{aligned}
$$

where $\mathbf{x}$ and $\mathbf{z}$ are variables.

The augmented Lagrangian of (2.14) is

$$
L_{\mu}(\mathbf{x}, \mathbf{z}, \boldsymbol{\lambda})=f(\mathbf{x})+g(\mathbf{z})+\boldsymbol{\lambda}^{T}(\mathbf{A} \mathbf{x}+\mathbf{B} \mathbf{z}-\mathbf{c})+\frac{\mu}{2}\|\mathbf{A x}+\mathbf{B} \mathbf{z}-\mathbf{c}\|_{2}^{2},
$$

where $\boldsymbol{\lambda}$ is the Lagrangian multiplier and $\mu$ is the Lagrangian penalty coefficient. To be differentiated with the penalty coefficient we used later, parameter $\mu$ will be called the Lagrangian coefficient. The quadratic term makes the dual function of $L_{\mu}(\mathbf{x}, \mathbf{z}, \boldsymbol{\lambda})$ be differentiable under rather mild conditions [34].

Now the constrained optimization problem turns into an unconstrained problem by minimizing the $L_{\mu}(\mathbf{x}, \mathbf{z}, \boldsymbol{\lambda})$. We can solve this by the following iterative operations: 


$$
\begin{aligned}
& \mathbf{x}^{k+1}=\arg \min _{\mathbf{x}} L_{\mu}\left(\mathbf{x}, \mathbf{z}^{k}, \boldsymbol{\lambda}^{k}\right), \\
& \mathbf{z}^{k+1}=\arg \min _{\mathbf{z}} L_{\mu}\left(\mathbf{x}^{k+1}, \mathbf{z}, \boldsymbol{\lambda}^{k}\right), \\
& \boldsymbol{\lambda}^{k+1}=\boldsymbol{\lambda}^{k}+\mu\left(\mathbf{A} \mathbf{x}^{k+1}+\mathbf{B} \mathbf{z}^{k+1}-\mathbf{c}\right) .
\end{aligned}
$$

In one iteration, the Lagrangian $L_{\mu}(\mathbf{x}, \mathbf{z}, \boldsymbol{\lambda})$ is minimized in terms of variable $\mathbf{x}$ with $\mathbf{z}$ and $\boldsymbol{\lambda}$ fixed. Then the Lagrangian is minimized in terms of variable $\mathbf{z}$ with $\mathbf{x}$ and $\boldsymbol{\lambda}$ fixed. Finally, $\boldsymbol{\lambda}$ is updated based on new values of $\mathbf{x}$ and $\mathbf{z}$. These three variables are updated alternatively. So the method is called the alternating direction method of multipliers.

It has been proved that for a convex optimization problem, the ADMM technique can give the global minimum. More importantly, for non-convex optimization, the ADMM technique can still be used to obtain a local minimum efficiently [34].

\subsubsection{ADMM Decoding Model}

Problem (2.13) is a convex optimization problem. So the ADMM technique can be used to solve (2.13) instead of the general LP solver. The solution given by the ADMM technique will be the global minimum. Therefore, ADMM decoding still preserves the "ML certificate" property.

The solution of the ADMM technique will converge to the global minimum.

Barman et al. rewrote (2.13) as follows [33]:

$$
\begin{aligned}
\operatorname{minimize} & \boldsymbol{\gamma}^{T} \mathbf{x} \\
\text { subject to } & \mathbf{P}_{j} \mathbf{x}=\mathbf{z}_{j}, \quad \forall j \in \mathcal{J}, \\
& \mathbf{z}_{j} \in \mathbb{P P}_{d_{j}}, \quad \forall j \in \mathcal{J},
\end{aligned}
$$

where $\mathbf{z}_{j}$ is a vector of length $d_{j}$. Then $\mathbf{z}_{j}$, instead of $\mathbf{P}_{j} \mathbf{x}$, is constrained by the parity-check equation of check node $j$. The introduction of $\mathbf{z}_{j}$ makes the model fit the standard template of the ADMM technique.

The augmented Lagrangian of (2.19) is

$$
L_{\mu}(\mathbf{x}, \mathbf{z}, \boldsymbol{\lambda})=\gamma^{T} \mathbf{x}+\sum_{j \in \mathcal{J}} \boldsymbol{\lambda}_{j}^{T}\left(\mathbf{P}_{j} \mathbf{x}-\mathbf{z}_{j}\right)+\frac{\mu}{2} \sum_{j \in \mathcal{J}}\left\|\mathbf{P}_{j} \mathbf{x}-\mathbf{z}_{j}\right\|_{2}^{2},
$$


which is still constrained by $\mathbf{x} \in[0,1]^{n}$ and $\mathbf{z}_{j} \in \mathbb{P P}_{d_{j}}$.

The scaled form of the ADMM technique solves this model by the following updating operations [39]:

$$
\begin{aligned}
\mathbf{x}^{k+1} & =\underset{\mathbf{x} \in[0,1]^{n}}{\arg \min }\left(\gamma^{T} \mathbf{x}+\frac{\mu}{2} \sum_{j \in \mathcal{J}}\left\|\mathbf{P}_{j} \mathbf{x}-\mathbf{z}_{j}^{k}+\boldsymbol{\lambda}_{j}^{k}\right\|_{2}^{2}\right), \\
\mathbf{z}_{j}^{k+1} & =\prod_{\mathbb{P}_{d_{j}}}\left(\mathbf{P}_{j} \mathbf{x}^{k+1}+\boldsymbol{\lambda}_{j}^{k}\right), \\
\boldsymbol{\lambda}_{j}^{k+1} & =\boldsymbol{\lambda}_{j}^{k}+\left(\mathbf{P}_{j} \mathbf{x}^{k+1}-\mathbf{z}_{j}^{k+1}\right),
\end{aligned}
$$

where $\prod_{\mathbb{P}_{d_{j}}}$ is the Euclidean projection onto check polytope $\mathbb{P P}_{d_{j}}$ and $\boldsymbol{\lambda}_{j}$ are Lagrangian multipliers. $\mu$ is the constant Lagrangian coefficient that can be selected based on the FER performance. $\mathbf{z}_{j}^{0}$ and $\boldsymbol{\lambda}_{j}^{0}$ can be initialized as vector $\mathbf{0 . 5}$ and vector $\mathbf{0}$, respectively.

More specifically, for model (2.19), the update of $\mathbf{x}$ can be solved and split into bit-wise operations:

$$
x_{i}^{k+1}=\Pi_{[0,1]} \frac{1}{d_{i}}\left(\sum_{j \in N_{v}(i)}\left(z_{j(i)}^{k}-\lambda_{j(i)}^{k}\right)-\frac{1}{\mu} \gamma_{i}\right),
$$

where $\Pi_{[0,1]}$ is the Euclidean projection onto the interval $[0,1], z_{j(i)}$ and $\lambda_{j(i)}$ denote the components of $\mathbf{z}_{j}$ and $\boldsymbol{\lambda}_{j}$ corresponding to $v_{i}$, respectively.

It should be noted the check polytope projection $\prod_{\mathbb{P P}_{d_{j}}}$ in the update of $\mathbf{z}_{j}$ is the most time-consuming operation in ADMM decoding of LDPC codes ${ }^{1}$. Barman et al. originally proposed a Two-Slice algorithm based on the finding that two adjacent slices can represent any points in the check polytope. Later several more efficient check polytope projection algorithms were proposed to simplify the complexity of ADMM decoding. We also propose an iterative check polytope projection algorithm in Chapter 3. So the details of the check polytope projection will be given there.

The ADMM decoding algorithm can be regarded as a message-passing algorithm. The message $x_{i}$ is updated at variable node $i$ and passed to its neighboring check nodes. The messages $\mathbf{z}_{j}$ and $\boldsymbol{\lambda}_{j}$ are updated at check node $j$ by the received variable node messages and the stored values in the previous iteration. Because of this

\footnotetext{
${ }^{1}$ Based on our simulations, even with the relatively efficient projection algorithm [39], the average time for check polytope projection operation is more than half of the total decoding time for the $(576,288)$ WiMAX code.
} 
message-passing characteristic, some classical techniques can be used to accelerate the convergence of the ADMM decoding algorithm, such as over-relaxation, early termination, and scheduling schemes.

During one ADMM decoding iteration, the updates of $\mathbf{z}$ and $\boldsymbol{\lambda}$ use the latest value of $\mathbf{x}$. For over-relaxation, the updates of $\mathbf{z}$ and $\boldsymbol{\lambda}$ do not directly use the latest value of $\mathbf{x}$. Instead, $\mathbf{P}_{j} \mathbf{x}^{k+1}$ is replaced by

$$
\omega^{k} \mathbf{P}_{j} \mathbf{x}^{k+1}+\left(1-\omega^{k}\right) \mathbf{z}_{j}^{k},
$$

where $\omega^{k} \in(1,2)$ is the over-relaxation coefficient. The over-relaxed ADMM decoding is similar to the accelerated ADMM framework proposed in [99], and a comparison between them can be found in [100].

Early termination is widely used in the iterative decoding algorithm. It means after each iteration, the hard-decision values of the variables are obtained and used to perform parity checking. If the parity check results are all zeros, the iterative procedure can be terminated in advance. Early termination can avoid unnecessary iteration when the decoding approaches convergence, while some of the variable values are still not integers.

The scheduling of messages can substantially influence the convergence of the ADMM decoding algorithm. The most commonly used scheduling scheme for the ADMM decoding algorithm is the flooding scheme. Layered scheduling was proposed in [51] to accelerate the convergence. We also propose a scheduling scheme in Chapter 4. So the details of the scheduling schemes will be provided there.

ADMM decoding is based on the LP decoding model relaxed from the IP decoding model. The relaxation of the IP decoding model makes the decoding problem feasible but also introduces fractional points to the feasible region. The factional points are called pseudo-codewords and degrade the error correction performance. To penalize pseudo-codewords, ADMM VN-penalized decoding algorithms [66-68] were proposed by adding penalty terms to the objective function of the LP decoding model. For example, ADMM VN-penalized decoding algorithm with $\ell_{2}$ penalty terms, $h_{2}(x)=$ $-\alpha(x-0.5)^{2}$, significantly improved the FER performance in the low SNR region with only the updating rule variable $x_{i}$ changed as follows:

$$
x_{i}^{k+1}=\Pi_{[0,1]} \frac{1}{d_{i}-\frac{2 \alpha}{\mu}}\left(\sum_{j \in N_{v}(i)}\left(z_{j(i)}^{k}-\lambda_{j(i)}^{k}\right)-\frac{\gamma_{i}}{\mu}-\frac{\alpha}{\mu}\right) .
$$


We propose an ADMM CN-penalized decoding algorithm in Chapter 5 and will show the details of the ADMM VN-penalized decoding algorithms there. 


\section{Chapter 3}

\section{Iterative Check Polytope Projection Algorithm}

As found in Section 2.3, the check polytope projection $\prod_{\mathbb{P P}_{d_{j}}}$ in the update of $\mathbf{z}_{j}$ is the most complex operation in ADMM decoding of LDPC codes. To simplify the ADMM decoding algorithm, it is crucial to simplify this bottle-neck operation. As we know, all previous projection algorithms require sorting or partial sorting operations, which introduce the majority of the complexity and prevent efficient hardware implementation. Thus, it is necessary to avoid the sorting operation. To indicate the general check polytope projection operation, the notation $d_{j}$ will be replaced by $d$ in this chapter.

In this chapter, we first review the check polytope and the cut search algorithm (CSA) based projection algorithm in Section 3.1. Then we propose an iterative check polytope projection algorithm that does not involve the complex operation of sorting in Section 3.2 and prove that the projection algorithm converges as the iterations increase. The computational complexity of the proposed algorithm and other algorithms are analyzed in Section 3.3. The simulation results are given in Section 3.4.

\subsection{Check Polytope and CSA-based Projection}

The check polytope projection operation $\prod_{\mathbb{P P}_{d}}(\mathbf{v})$ is the operation to find the nearest point in the check polytope $\mathbb{P P}_{d}$ to the given point $\mathbf{v} \in \mathbb{R}^{d}$. Mathematically, the check polytope $\mathbb{P P}_{d}$ of dimension $d$ can be described by a set of box constraints and parity inequalities as follows [39]: 


$$
\begin{aligned}
& 0 \leq u_{i} \leq 1, \forall i \in[d] \\
& \boldsymbol{\theta}_{V}^{T} \mathbf{u} \leq p_{V}, \forall V \subseteq[d] \text { with }|V| \text { odd }
\end{aligned}
$$

where $[d]$ is defined as the set of integers from 1 to $d, \boldsymbol{\theta}_{V}$ is the indicator vector of set $V, V^{c}$ is the complement set $[d] \backslash V$, and $p_{V}=|V|-1$. The components of $\boldsymbol{\theta}_{V}$ are

$$
\theta_{V, i}= \begin{cases}1 & \text { if } i \in V \\ -1 & \text { if } i \in V^{c}\end{cases}
$$

The box constraints (3.1) show that the check polytope $\mathbb{P P}_{d}$ lies inside the unit hypercube, i.e., $\mathbb{P P}_{d} \subseteq[0,1]^{d}$. The parity inequalities (3.2) define several half-spaces. For a point $\mathbf{u} \in[0,1]^{d}$, if $\boldsymbol{\theta}_{V}^{T} \mathbf{u}>p_{V}$ for one set $V \subseteq[d]$ of odd cardinality, $\mathbf{u} \notin \mathbb{P P}_{d}$. This inequality is called a cut at $\mathbf{u}$. On the other side, if $\boldsymbol{\theta}_{V}^{T} \mathbf{u} \leq p_{V}$ for all sets $V \subseteq[d]$ of odd cardinality, the point $\mathbf{u}$ lies inside (or on) $\mathbb{P P}_{d}$.

Among all parity inequalities, for a point $\mathbf{u} \in[0,1]^{d}$, an inequality $\boldsymbol{\theta}^{T} \mathbf{x} \leq p$ can be found by the CSA in [40]. It has been proved that if this inequality is satisfied, then all the other parity inequalities must also be satisfied [40]. This inequality is called the most vulnerable inequality. If the inequality sign is replaced by the equality sign, a hyperplane $\boldsymbol{\theta}^{T} \mathbf{x}=p$ is defined, and it is termed the assistant hyperplane in this chapter.

Proposition 1 (Proposition 1 in [39]). If at any given point $\mathbf{u} \in[0,1]^{d}$, one of the parity inequalities in (3.2) is violated, then the rest of them are satisfied with strict inequality.

Proposition 1 shows that not all parity inequalities are essential to judge if a point is also inside the check polytope when it is inside the unit hypercube. For a point inside the unit hypercube, the most vulnerable inequality found by the CSA should be verified first. If this inequality is not satisfied, then the other parity inequalities must be satisfied. So it will be a waste if we verify the other parity inequalities first. On the other hand, if this inequality is satisfied, the other parity inequalities must also be satisfied, as mentioned before. As a result, there is no need to check the other inequalities after the most vulnerable inequality is verified. It also implies that there exists at most one cut for the given point. 
In the CSA-based projection algorithm, three steps are used to get the check polytope projection result $\mathbf{z}=\prod_{\mathbb{P}_{d}}(\mathbf{v})$.

Firstly, the point $\mathbf{v} \in \mathbb{R}^{d}$ is projected onto the hypercube. The projection onto the hypercube, $\mathbf{u}=\Pi_{[0,1]^{d}}(\mathbf{v})$, can be done component-wisely by

$$
u_{i}=v_{i} \mathrm{U}\left(v_{i}\right)-\left(v_{i}-1\right) \mathrm{U}\left(v_{i}-1\right)=\left\{\begin{array}{cc}
1 & , \text { if } v_{i}>1 \\
0 & , \text { if } v_{i}<0 \\
v_{i} & , \text { otherwise }
\end{array}\right.
$$

where $\mathrm{U}(x)$ is the unit step function.

Secondly, the assistant hyperplane $\boldsymbol{\theta}^{T} \mathbf{x}=p$ for point $\mathbf{u}$ is derived by the CSA with two steps. The first step is assigning the initial value to $\theta_{i}$ by

$$
\theta_{i}=\operatorname{sgn}\left(u_{i}-0.5\right)=\left\{\begin{aligned}
1 & , \text { if } u_{i}>1 / 2 \\
-1 & , \text { otherwise }
\end{aligned}\right.
$$

The second step is to find the number of ones in $\boldsymbol{\theta}$. If the number (for example $t$ ) is even, we have to flip the value of $\theta_{i}$ whose corresponding $u_{i}$ is closest to 0.5 and update $t$. After that, we have $p=t-1$. We note here that the assistant hyperplane for point $\mathbf{u}$ can also be calculated with $\mathbf{v}$ by the same steps without changing the result.

If this projection $\mathbf{u}=\Pi_{[0,1]^{d}}(\mathbf{v})$ is not "cut" by the assistant hyperplane, $\mathbf{u}$ is actually the projection onto the check polytope. If the hypercube projection is outside the check polytope, the cut found by the CSA can be further used to find the check polytope projection by Theorem 1 below in the final step.

Theorem 1 (Theorem 3 in [39]). For a given vector $\mathbf{v} \in \mathbb{R}^{d}$, let $\mathbf{u}=\prod_{[0,1]^{d}}(\mathbf{v})$. If $\boldsymbol{\theta}^{T} \mathbf{u}>p$ is a cut for $\mathbf{u}$, then $\prod_{\mathbb{P}_{d}}(v)$ must be on the facet of $\mathbb{P P}_{d}$ defined by the cut, i.e., $\prod_{\mathbb{P P}_{d}}(v) \in\left\{\mathbf{x} \in[0,1]^{d} \mid \boldsymbol{\theta}^{T} \mathbf{x}=p\right\}$.

By Theorem 1, the projection onto the check polytope can be converted to the projection onto the facet. The following optimization problem can be formulated to 
find the projection of a point $\mathbf{v}$ :

$$
\begin{aligned}
\operatorname{minimize} & \|\mathbf{z}-\mathbf{v}\|_{2}^{2} \\
\text { subject to } & 0 \leq z_{i} \leq 1, \forall i \in[d], \\
& \boldsymbol{\theta}^{T} \mathbf{z}=p
\end{aligned}
$$

The solution to the optimization problem is

$$
\mathbf{z}=\Pi_{[0,1]^{d}}(\mathbf{v}-s \boldsymbol{\theta}),
$$

where $s$ is a scalar (we call it difference coefficient) such that $\boldsymbol{\theta}^{T} \mathbf{z}=p$.

In the final step, the difference coefficient $s$ is calculated precisely by Algorithm 3 in [39] which involves a sorting operation.

\subsection{Iterative Polytope Projection Algorithm}

In [39], points are divided into two classes, $\Pi_{[0,1]^{d}}(\mathbf{v}) \in \mathbb{P P}_{d}$ and $\Pi_{[0,1]^{d}}(\mathbf{v}) \notin \mathbb{P P}_{d}$, with different projection rules. We can combine the two cases, using the same projection rule by the following proposition.

Proposition 2. For a given vector $\mathbf{v} \in \mathbb{R}^{d}$, let $\boldsymbol{\theta}^{T} \mathbf{x}=p$ be the assistant hyperplane of check polytope $\mathbb{P P}_{d}$. The projection of $\mathbf{v}$ onto $\mathbb{P P}_{d}$ is $\mathbf{z}=\Pi_{[0,1]^{d}}(\mathbf{v}-$ s $\boldsymbol{\theta})$, where $s$ is a scalar such that $\boldsymbol{\theta}^{T} \mathbf{z} \leq p$.

Proof. For $\Pi_{[0,1]^{d}}(\mathbf{v}) \notin \mathbb{P P}_{d}$, it is true by (3.7). For $\Pi_{[0,1]^{d}}(\mathbf{v}) \in \mathbb{P P}_{d}$, the projection result is $\Pi_{[0,1]^{d}}(\mathbf{v})$ that can be written as $\Pi_{[0,1]^{d}}(\mathbf{v}-0 \cdot \boldsymbol{\theta})$. In this case the difference coefficient $s$ is 0 . Since the projection result is inside or on the polytope, we have $\boldsymbol{\theta}^{T} \mathbf{z} \leq p$

Accordingly, we can derive a three-step projection algorithm. Firstly we get the assistant hyperplane $\boldsymbol{\theta}^{T} \mathbf{x}=p$. Secondly, we get the difference coefficient $s$. Finally, we get the projection by $\mathbf{z}=\Pi_{[0,1]^{d}}(\mathbf{v}-s \boldsymbol{\theta})$. We now focus on how to get the difference coefficient $s$ efficiently.

For $\mathbf{u}=\Pi_{[0,1]^{d}}(\mathbf{v}) \in \mathbb{P P}_{d}$, we have $\boldsymbol{\theta}^{T} \mathbf{u} \leq p$. Therefore we can calculate $\eta=\left(\boldsymbol{\theta}^{T} \mathbf{u}-p\right) / d$. If $\eta \leq 0$, the difference coefficient $s$ can be set to 0 . 
For $\mathbf{u}=\Pi_{[0,1]^{d}}(\mathbf{v}) \notin \mathbb{P P}_{d}$, we have $\eta>0$. Suppose $\mathbf{v}^{\prime}=\mathbf{v}-s \boldsymbol{\theta}$, where $s$ is the exact difference coefficient. We can use $\eta$ to approximate $s$ and update $\mathbf{v} \leftarrow \mathbf{v}-\eta \boldsymbol{\theta}$. For the new point $\mathbf{v}$, we can obtain the projection $\mathbf{u}=\Pi_{[0,1]^{d}}(\mathbf{v})$ and its $\eta=\left(\boldsymbol{\theta}^{T} \mathbf{u}-p\right) / d$. This procedure is executed iteratively until the updated $\mathbf{v}$ is close enough to $\mathbf{v}^{\prime}$ and $s$ is actually close to the summation of all $\eta$ we calculated. The principle behind this procedure is based on the following two propositions.

Proposition 3. For a given point $\mathbf{v} \in \mathbb{R}^{d}$ and a hyperplane $\boldsymbol{\theta}^{T} \mathbf{x}=p$, the projection of $\mathbf{v}$ on the hyperplane is $\mathbf{v}-\eta \boldsymbol{\theta}$, where $\eta=\left(\boldsymbol{\theta}^{T} \mathbf{v}-p\right) /\left(\boldsymbol{\theta}^{T} \boldsymbol{\theta}\right)$, called projection coefficient. If $\theta_{i}$ can only be 1 or -1 , then $\eta=\left(\boldsymbol{\theta}^{T} \mathbf{v}-p\right) / d$.

Proof. The proof of the first part is a simple linear algebra problem by solving two equations

$$
\left\{\begin{aligned}
\mathbf{v}-\mathbf{x} & =\eta \boldsymbol{\theta} \\
\boldsymbol{\theta}^{T} \mathbf{x} & =p,
\end{aligned}\right.
$$

where $\mathbf{x}$ is the projection point on the hyperplane. So $\eta=\left(\boldsymbol{\theta}^{T} \mathbf{v}-p\right) /\left(\boldsymbol{\theta}^{T} \boldsymbol{\theta}\right)$. If $\theta_{i}$ can only be 1 or -1 , then $\boldsymbol{\theta}^{T} \boldsymbol{\theta}=\|\boldsymbol{\theta}\|_{2}^{2}=d$.

Proposition 3 describes the relation between a point and a hyperplane. If a point is on the hyperplane, $\eta=0$. If a point is not on the hyperplane, the sign of $\eta$ indicates which side the point is, and the absolute value of $\eta$ shows the "relative distance" between the point and the hyperplane.

Proposition 4. For a given point $\mathbf{v} \in \mathbb{R}^{d}$ and the assistant hyperplane $\boldsymbol{\theta}^{T} \mathbf{x}=p$ of check polytope $\mathbb{P P}_{d}$, if $\mathbf{u}=\Pi_{[0,1]^{d}}(\mathbf{v}) \notin \mathbb{P P}_{d}$, the difference coefficient s of $\mathbf{v}$ satisfies $s \geq \eta=\left(\boldsymbol{\theta}^{T} \mathbf{u}-p\right) / d>0$.

Proof. The difference coefficient of $\mathbf{v}$ can be written as

$$
s=\left(\boldsymbol{\theta}^{T} \mathbf{u}-p+\Delta\right) /(d-k)
$$

where $\Delta \geq 0$ and $0 \leq k<d$. (3.9) is summarized from Algorithm 3 of [39], and the exact value of $\Delta$ and $k$ is determined based on a sorting operation. It is obvious that the difference coefficient $s$ of $\mathbf{v}$ is equal to or larger than $\eta=\left(\boldsymbol{\theta}^{T} \mathbf{u}-p\right) / d$.

Since $\mathbf{v}^{\prime}=\mathbf{v}-s \boldsymbol{\theta}, \boldsymbol{\theta}$ and $s$ can be considered as the "direction" and the "relative distance" from $\mathbf{v}$ to $\mathbf{v}^{\prime}$. Proposition 4 presents the connection between the difference 


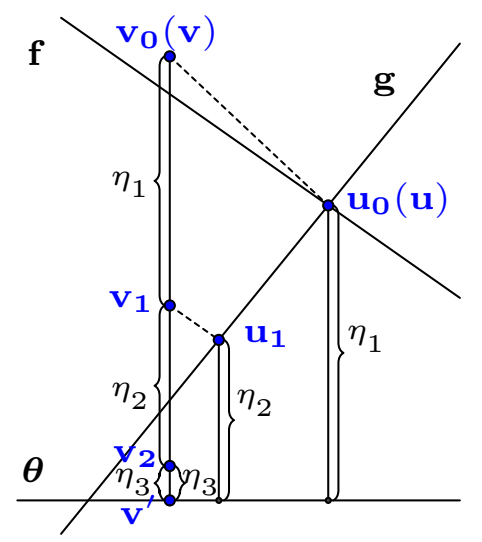

(a) Vector $\mathbf{v}_{0}=\mathbf{v}$ reaches $\mathbf{v}^{\prime}$ at Iteration 3 .

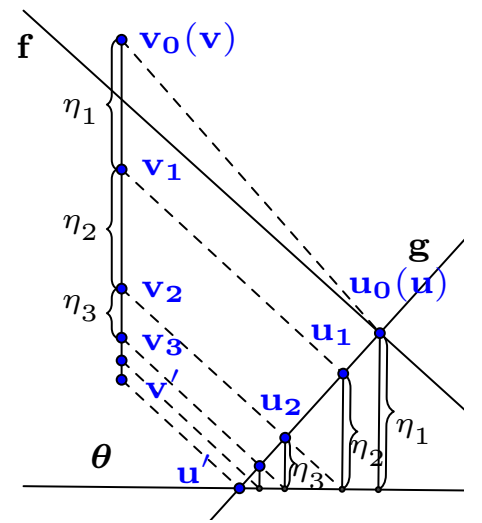

(b) Vector $\mathbf{v}_{k}$ converges to $\mathbf{v}^{\prime}$ as $k$ increases.

Figure 3.1: Illustration of the iterative check polytope projection. Vector $\boldsymbol{\theta}$ represents the assistant hyperplane. Notations $\mathbf{f}$ and $\mathbf{g}$ are used to indicate the boundaries of the unit hypercube. Dash lines represent projection onto the hypercube.

coefficient $s$ of $\mathbf{v}$ and projection coefficient $\eta$ of $\mathbf{u}=\Pi_{[0,1]^{d}}(\mathbf{v})$. That is the distance from $\mathbf{v}$ to $\mathbf{v}^{\prime}$ is always equal to or larger than the distance from $\mathbf{u}$ to the assistant hyperplane. If we approximate $s$ by $\eta$ and update $\mathbf{v} \leftarrow \mathbf{v}-\eta \boldsymbol{\theta}$, the point $\mathbf{v}$ will move towards $\mathbf{v}^{\prime}$ but never go beyond it. Now the distance from $\mathbf{v}$ to $\mathbf{v}^{\prime}$ becomes shorter and it can again be approximated by $\eta=\left(\boldsymbol{\theta}^{T} \mathbf{u}-p\right) / d$, where $\mathbf{u}$ is the projection point of the new $\mathbf{v}$ onto hypercube. It should be noted that the new distance is still equal to or larger than $\eta$. Therefore, after enough iterations, $\mathbf{v}$ will be close enough to $\mathbf{v}^{\prime}$ and the original difference coefficient $s$ can be approximated by the summation of all $\eta$ we calculated.

It should be noted that Proposition 4 is equivalent to the following form which is summarized by Dr. Amir Banihashemi in [46]. 
Proposition 5. Consider a vector $\mathbf{v} \in \mathbb{R}^{d}$ with the assistant hyperplane $\boldsymbol{\theta}^{T} \mathbf{x}=p$ for the check polytope $\mathbb{P P}_{d}$, and the difference coefficient s. If $\mathbf{u}=\Pi_{[0,1]^{d}}(\mathbf{v}) \notin \mathbb{P P}_{d}$, then, in the proposed iterative check polytope projection algorithm, the projection coefficient $\eta_{i}=\left(\boldsymbol{\theta}^{T} \mathbf{u}_{i-1}-p\right) / d$ satisfies $\eta_{i} \leq s-\sum_{j=1}^{i} \eta_{j-1}$, for any $i \geq 1$, where $\mathbf{u}_{i-1}=$ $\Pi_{[0,1]^{d}}\left(\mathbf{v}-\left(\sum_{j=1}^{i} \eta_{j-1}\right) \boldsymbol{\theta}\right)$.

Proposition 5 shows more clearly that, at each iteration of the proposed projection algorithm, vector $\mathbf{v}$ is shifted closer and closer to the desired vector $\mathbf{v}^{\prime}\left(\eta_{i}>0\right)$, but will never pass $\mathbf{v}^{\prime}$ since $\eta_{i} \leq s-\sum_{j=1}^{i} \eta_{j-1}$. It thus follows that, by increasing the number of iterations, the sequence of shifted versions of $\mathbf{v}$ either reaches or converges to $\mathbf{v}^{\prime}$, and therefore Algorithm 1 either reaches or converges to vector $\mathbf{z}=\prod_{\mathbb{P P}_{d}}(\mathbf{v})$. The formal convergence analysis is given below.

Proposition 6. Consider a vector $\mathbf{v} \in \mathbb{R}^{d}$ with the assistant hyperplane $\boldsymbol{\theta}^{T} \mathbf{x}=p$ for the check polytope $\mathbb{P P}_{d}$, and the difference coefficient s. If $\mathbf{u}=\Pi_{[0,1]}(\mathbf{v}) \notin \mathbb{P P}_{d}$, then, in the proposed iterative check polytope projection algorithm, $\mathbf{u}_{i}=\Pi_{[0,1]^{d}}(\mathbf{v}-$ $\left.\left(\sum_{j=1}^{i} \eta_{j}\right) \boldsymbol{\theta}\right) \rightarrow \Pi_{[0,1]^{d}}(\mathbf{v}-s \boldsymbol{\theta})$ as $i \rightarrow \infty$, where $\eta_{i}=\left(\boldsymbol{\theta}^{T} \mathbf{u}_{i-1}-p\right) / d$.

Proof. It is true because $\eta_{i} \leq s-\sum_{j=1}^{i} \eta_{j-1}$ and $\eta_{i}>0$ for any $i \geq 1$.

We first show $\eta_{i} \leq s-\sum_{j=1}^{i} \eta_{j-1}$ for any $i \geq 1$ by induction. From Proposition 4 , for $i=1$, this inequality holds. If the inequality holds for an arbitrary value of $i \geq 1$, then $\eta_{i} \leq s-\sum_{j=1}^{i} \eta_{j-1}$ or $\sum_{j=1}^{i+1} \eta_{j-1} \leq s$. Therefore, the shifted vector $\mathbf{v}_{\mathbf{i}}=\mathbf{v}-\sum_{j=1}^{i+1} \eta_{j-1} \boldsymbol{\theta}$ will not pass $\mathbf{v}^{\prime}=\mathbf{v}-s \boldsymbol{\theta}$. Since $s$ is the scalar that makes $\boldsymbol{\theta}^{T} \mathbf{u}^{\prime}=p$, where $\mathbf{u}^{\prime}=\Pi_{[0,1]^{d}}\left(\mathbf{v}^{\prime}\right)$, then $\boldsymbol{\theta}^{T} \mathbf{u}_{\mathbf{i}}>p$. This means that $\boldsymbol{\theta}^{T} \mathbf{x}=p$ is also the assistant hyperplane of $\mathbf{v}_{\mathbf{i}}$ and cuts $\mathbf{u}_{\mathbf{i}}$. It then follows that the difference coefficient of $\mathbf{v}_{\mathbf{i}}$ with respect to the assistant hyperplane is $s-\sum_{j=1}^{i+1} \eta_{j-1}$, and thus from (3.9 ), we have $\eta_{i+1} \leq s-\sum_{j=1}^{i+1} \eta_{j-1}$. This means that the claim of proposition holds for $i+1$, and thus by induction, it holds for any value of $i \geq 1$.

We can also show $\eta_{i}>0$ for any $i \geq 1$ because $\boldsymbol{\theta}^{T} \mathbf{u}_{\mathbf{i}}>p$.

Fig. 3.1 illustrates the procedure of iterative projection. $\mathbf{v}_{0}$ indicates the initial position of the input $\mathbf{v}$. The distance from $\mathbf{v}_{0}$ to $\mathbf{v}^{\prime}$ is denoted by $s_{1}$. By using $\eta_{1}=$ $\left(\boldsymbol{\theta}^{T} \mathbf{u}_{0}-p\right) / d$ which is the distance from $\mathbf{u}_{1}$ to $\boldsymbol{\theta}$ to approximate $s_{1}$, the projection position moves from $\mathbf{v}_{0}$ to $\mathbf{v}_{1}$. After that, we use $\eta_{2}=\left(\boldsymbol{\theta}^{T} \mathbf{u}_{1}-p\right) / d$ to approximate the difference coefficient of $\mathbf{v}_{1}$, where $\mathbf{u}_{1}=\Pi_{[0,1]^{d}}\left(\mathbf{v}_{1}\right)$. The projection position moves from $\mathbf{v}_{1}$ to $\mathbf{v}_{2}$. When this procedure is repeated, the new projection position arrives or gets closer and closer to $\mathbf{v}^{\prime}$. The projection result is $\mathbf{u}=\Pi_{[0,1]}\left(\mathbf{v}_{k}\right)$. In Fig. 3.1a, 
the projection point $\mathbf{v}_{3}$ reaches $\mathbf{v}^{\prime}$ at Iteration 3 and in Fig. $3.1 \mathrm{~b}$, the projection point $\mathbf{v}_{k}$ converges to $\mathbf{v}^{\prime}$ as $k$ increases. The details of the iterative check polytope projection algorithm is as follows.

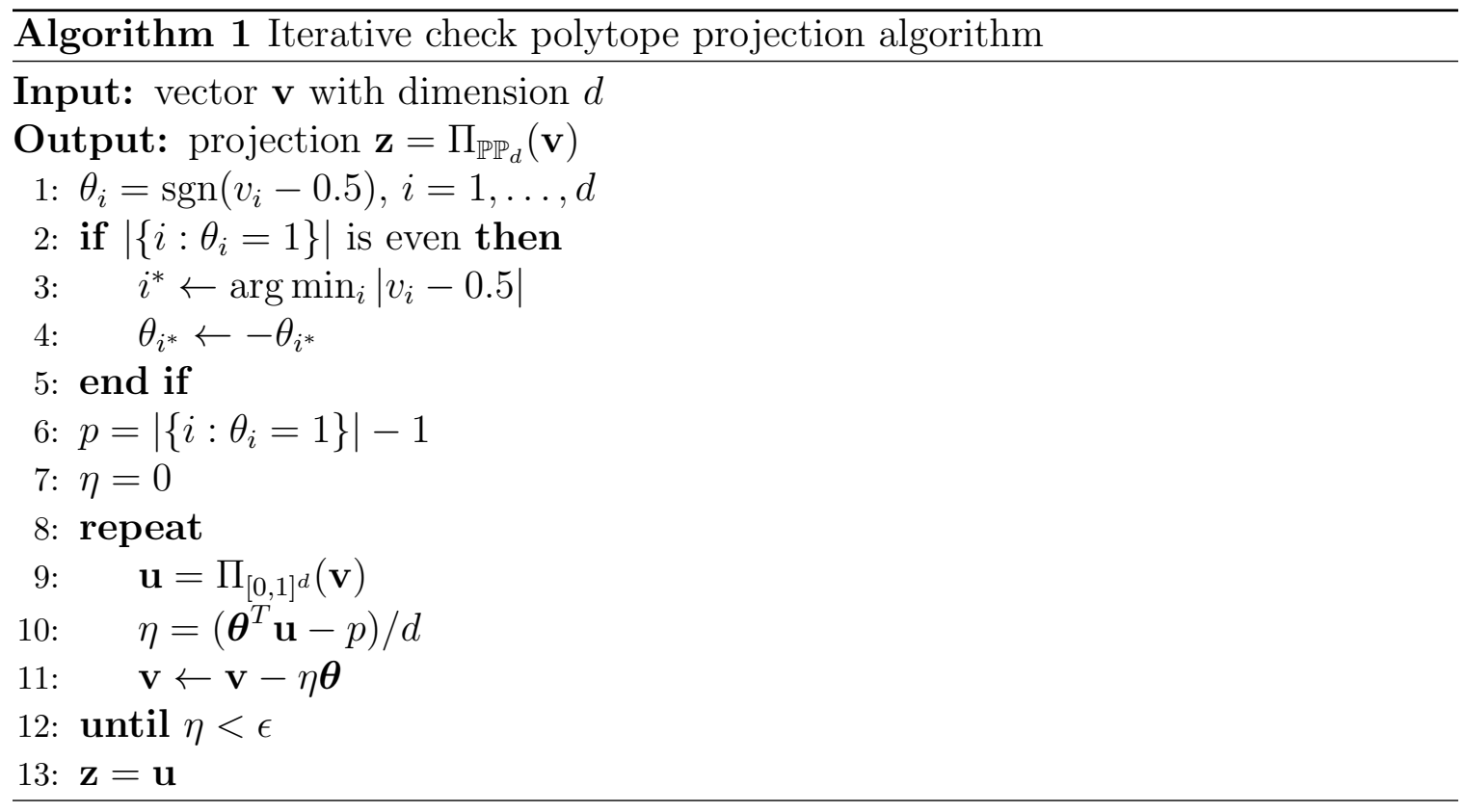

In Algorithm 1, the assistant hyperplane is found in Lines 1-6, which is the common step for [39]. For $\Pi_{[0,1]^{d}}(\mathbf{v}) \in \mathbb{P P}_{d}$, only one iteration is required. The algorithm stops when the projection coefficient $\eta$ less than the threshold $\epsilon$. To reduce the complexity, the maximum number of iterations $I_{\max }$ can be defined and used to limit the number of iterations.

\subsection{Complexity Analysis}

In this section, we compare the proposed projection algorithm with previous projection algorithms. As mentioned in [42], the partial sorting operation which is the main reason why the projection algorithm in [41] has lower complexity than [33] and [39] cannot be implemented in hardware efficiently. So, the projection algorithms compared here are the CSA-based projection algorithm in [39] and Wasson's projection in [42] which considers the FPGA implementation. The proposed projection algorithm is much simpler than the other two algorithms in three aspects.

First of all, points are divided into two classes in the CSA-based and Wasson's projection algorithms with different updating rules (especially for Wasson's algorithm). 
This classification demands designing two hardware components for two different projection rules, but each time only one of them will be used. In contrast, the unified updating rule in the proposed algorithm allows the hardware component to be fully used.

Secondly, the CSA-based and Wasson's projection algorithms use the sign of $\boldsymbol{\theta}^{T} \mathbf{u}-$ $p$ to judge if a point in the unit hypercube also lies in check polytope. If the point lies outside the check polytope, this value will not be used anymore. However, in our algorithm, we use the sign of $\eta=\left(\boldsymbol{\theta}^{T} \mathbf{u}-p\right) / d$ to make the same decision, and $\eta$ could be used again if the point lies outside the check polytope.

Last but not least, the proposed algorithm does not involve the sorting operation which is the bottle-neck of the previous projection algorithm. The average complexity and the worst-case complexity of the proposed algorithm are both linear in the input dimension because of removing the sorting operation. While for the other two algorithms, the average complexities are all $O(d \log (d))$, and the worst-case complexities are all $O\left(d^{2}\right)$. Moreover, the hardware consumption for implementing arithmetic operations is much lower than the sorting operation. This boosts the potential for deploying the ADMM decoder in reality.

\subsection{Simulation Results}

We implement the simulation program in C language. All simulations are performed over the AWGN channel with BPSK modulation. The ADMM decoding algorithm used here is actually the ADMM penalized decoding algorithm with $\ell_{2}$ penalty terms [66] and the penalty parameter $\alpha$ is 1.7. In the algorithm, the Lagrangian parameter $\mu$ is set to 2.8 , and the over-relaxation coefficient is set to 1.8. The maximum iteration number for ADMM decoding is selected to be 60. For each SNR point, the Monte Carlo simulation is performed, and 100 decoding failures are collected to estimate the FER with a quite tight confidence interval. ${ }^{1}$

In the first experiment, we use the irregular $(576,288)$ rate- $1 / 2$ code $\mathcal{C}_{1}$ from

\footnotetext{
${ }^{1}$ In our case, the simulation gives a binomially-distributed observation and its $95 \%$ confidence interval is $\left[\hat{p}-1.96 \sqrt{\frac{\hat{p}(1-\hat{p})}{n}}, \hat{p}+1.96 \sqrt{\frac{\hat{p}(1-\hat{p})}{n}}\right]$, where $\hat{p}$ is the estimate of the FER and $n$ is the total number of frames. By collecting 100 decoding failures, we have $n=100 / \hat{p}$ and then $1.96 \sqrt{\frac{\hat{p}(1-\hat{p})}{n}}=$ $1.96 \sqrt{\frac{\hat{p}^{2}(1-\hat{p})}{100}}=0.196 \hat{p} \sqrt{(1-\hat{p})}<0.196 \hat{p}$. The confidence interval is roughly $[0.8 \hat{p}, 1.2 \hat{p}]$, which is sufficiently tight for the purpose of this research.
} 


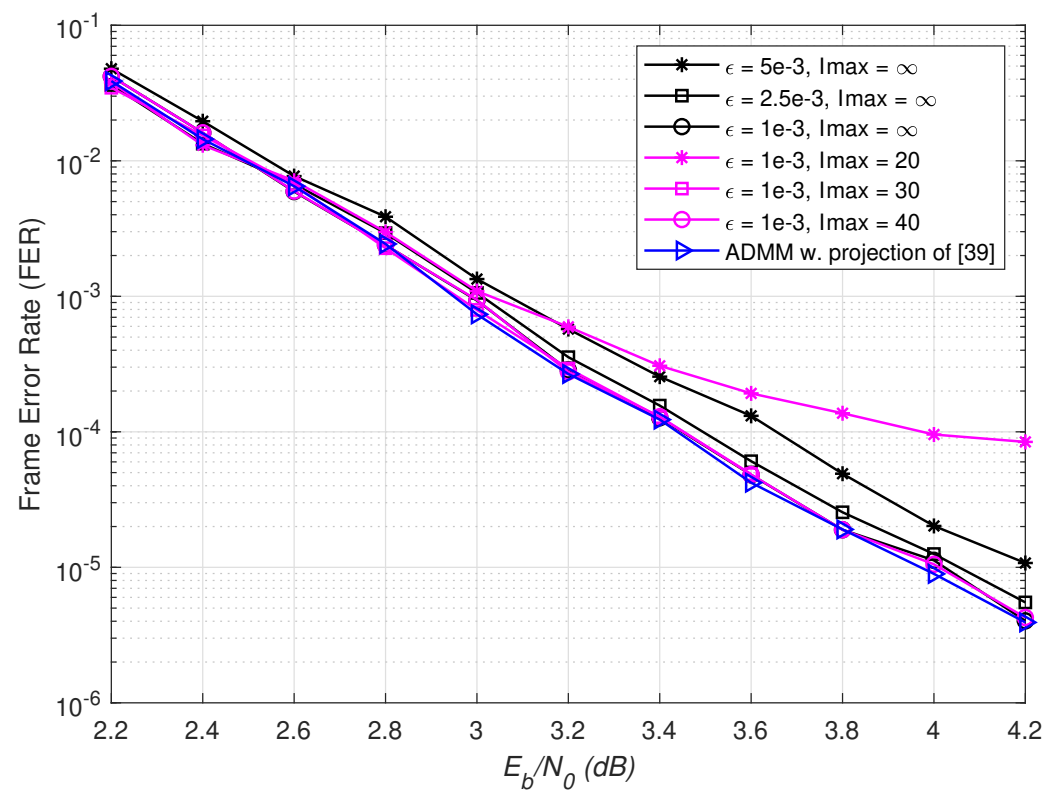

Figure 3.2: FER performance of $\mathcal{C}_{1}$ under ADMM decoding with the proposed check polytope projection algorithm for different values of $\epsilon$ and $I_{\max }$.

IEEE 802.16e (WiMAX) standard [4]. $\mathcal{C}_{1}$ has two check node degrees, 6 and 7. Fig. 3.2 shows the FER performance of ADMM decoding with the proposed algorithm and the accurate algorithm of [39] for the check polytope projection. The maximum number of iterations of the proposed projection algorithm is either limited by $I_{\max }$ or can be infinitely large. For each simulation point, 100 frame errors are obtained. It can be seen that the performance of the proposed projection algorithm depends on $\epsilon$ and $I_{\max }$. The performance can be improved by decreasing $\epsilon$ or by increasing $I_{\max }$. A reasonable selection of $\epsilon$ and $I_{\max }$ not only provides projection results with necessary accuracy but also avoids the unnecessary iterations. It can be seen that the FER performance of the proposed algorithm for $\epsilon=0.001$ with $I_{\max }=30$ is almost identical to that of [39] in the simulated range of $E_{b} / N_{0}$.

Fig. 3.3 gives the distributions of projections during one ADMM decoding with the proposed check polytope projection algorithm for different parameters based on the iteration numbers. We can see that the majority of the projections only use one iteration to get the projection result in all nine cases, and this percentage increases with $E_{b} / N_{0}$ values for the same $\epsilon$. For the projection algorithm with $\epsilon=2.5 e-3$, about $40 \%$ of the total projections need one iteration to get the result when $E_{b} / N_{0}=3.0$ $\mathrm{dB}$. The percentage increases to around $55 \%$ when $E_{b} / N_{0}=4.2 \mathrm{~dB}$. It is also shown 

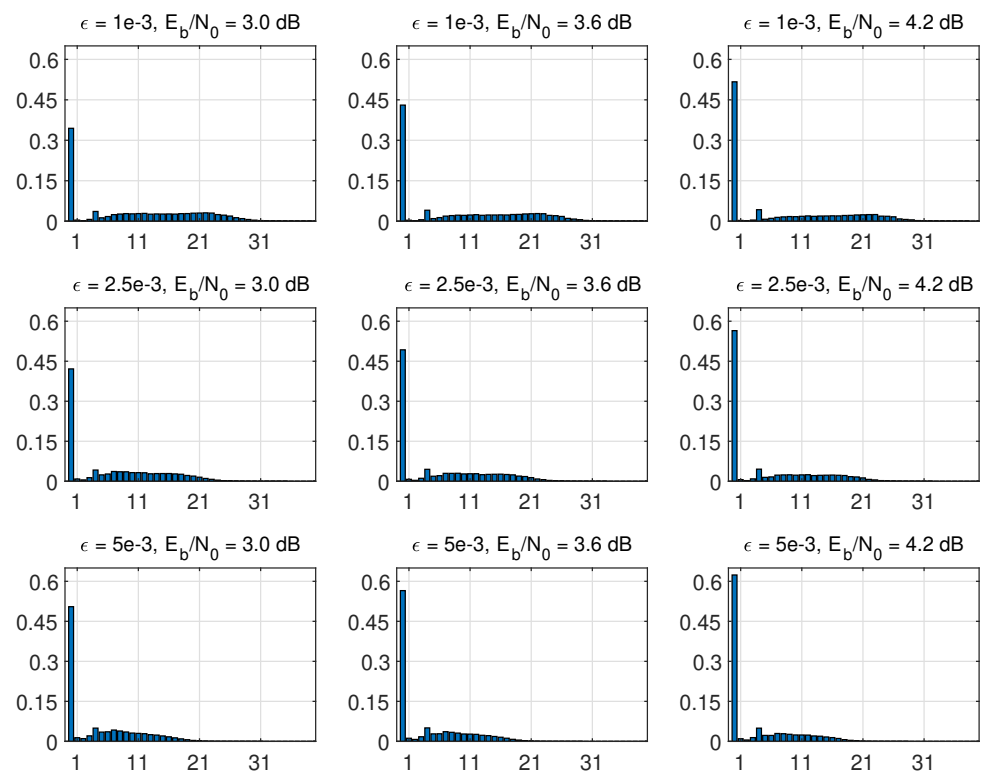

Figure 3.3: Distributions of the number of projection iterations during ADMM decoding with the proposed check polytope projection algorithm for different parameters.

that at the same $E_{b} / N_{0}$ value, the projection algorithm with a larger $\epsilon$ needs fewer iterations to get the result.

We also plot the histogram of average iteration numbers of the proposed projection algorithms in Fig. 3.4. From the figure, we can find the average number of iterations decreases with $E_{b} / N_{0}$ for all 3 parameter settings. For the projection algorithm with $\epsilon=2.5 e-3$, it only takes around 7 iterations on average to get the result when $E_{b} / N_{0}=4.2 \mathrm{~dB}$. Apart from that, the projection algorithm with a larger $\epsilon$ needs fewer iterations to get the result, but in high $E_{b} / N_{0}$, the differences become small, which explains why the average decoding time under ADMM decoding with the three parameter settings tends to be close.

More experiments about the maximum number of iterations are carried out on five randomly constructed regular LDPC codes, $\mathcal{C}_{2}-\mathcal{C}_{6}$, in Fig. 3.5. They are all of length 1000 (except for $\mathcal{C}_{3}$ which is of length 999) and all with variable node degree 3. Their check node degree is $6,9,12,15$, and 30 , respectively. The proposed projection algorithm terminates only if the maximum number of iterations is reached. It can be seen that although the average number of iterations is small, the proposed projection 


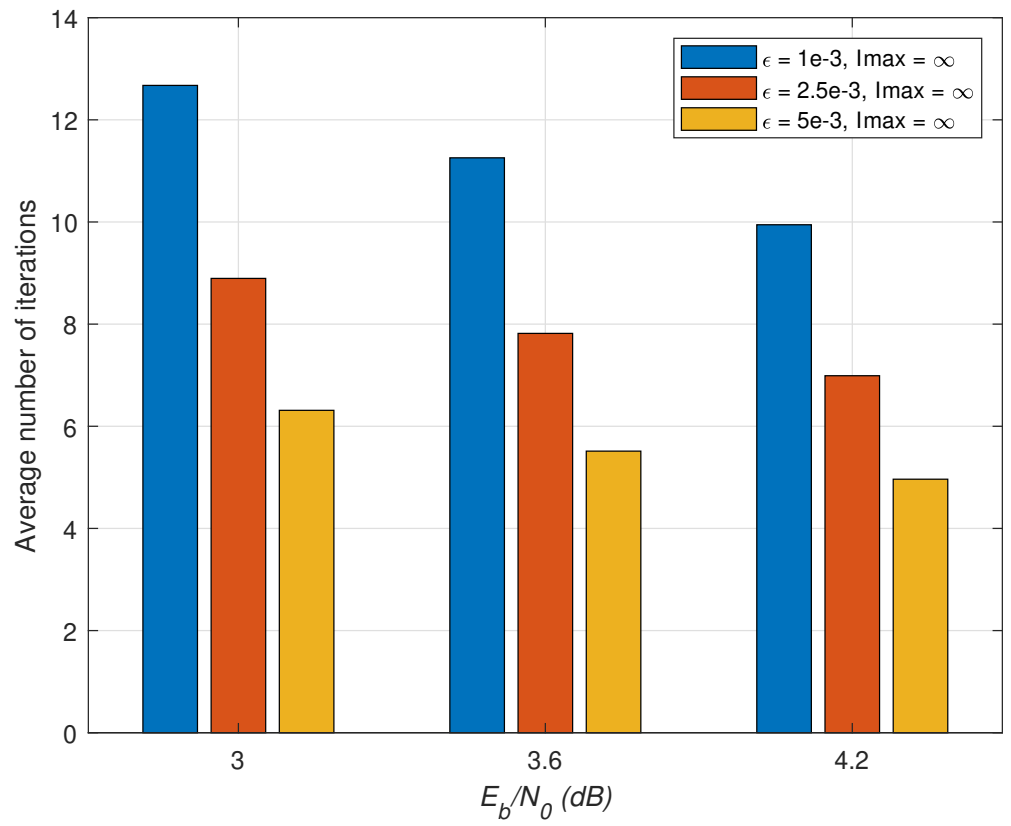

Figure 3.4: Average iteration number of the proposed check polytope projection algorithm for different parameters.

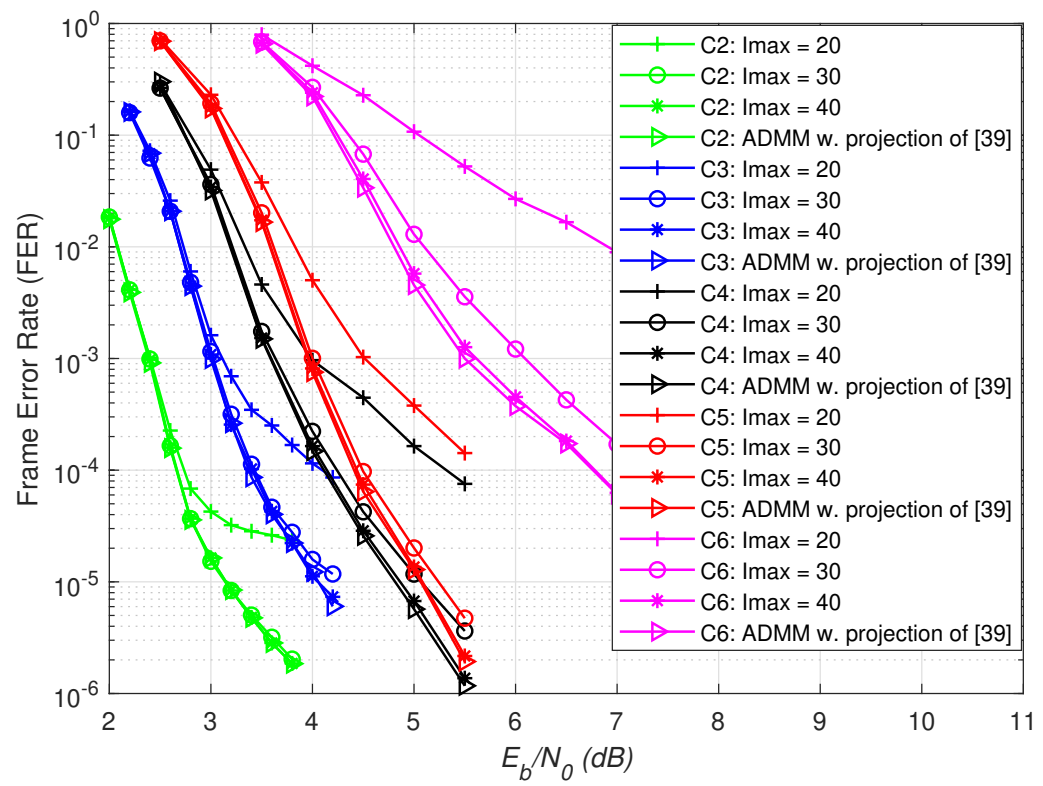

Figure 3.5: FER performance of $\mathcal{C}_{2}-\mathcal{C}_{6}$ under ADMM decoding with the proposed check polytope projection algorithm for different values of $I_{\max }$. 


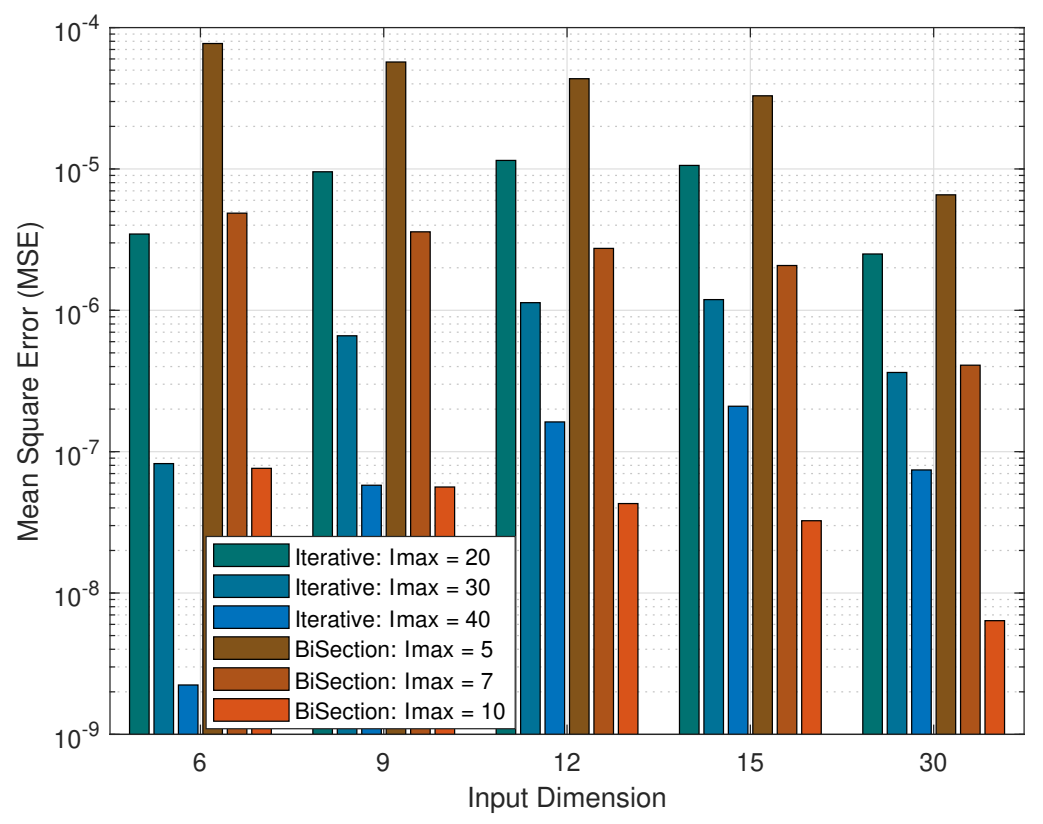

Figure 3.6: MSE of the projection result for the proposed check polytope projection algorithm and the bisection projection algorithm for different values of input dimension and $I_{\max }$.

should not be limited to a small number of iterations. The proposed projection algorithm needs enough iterations to converge, and a too-small iteration number will degrade the FER performance. For the simulated range, $I_{\max }=30$ and $I_{\max }=40$ are sufficient for $\mathcal{C}_{2}$ and $\mathcal{C}_{3}-\mathcal{C}_{6}$, respectively.

It has been reported in [47] that the proposed iterative projection algorithm takes a long time to converge. The authors came up with the idea of using the bisection search algorithm to find the difference coefficient $s$. The lower bound and the upper bound they used are 0 and $\left(\min _{i \in V} v_{i}-\max _{j \in V^{c}} v_{j}\right) / 2$, respectively, where $V$ is the indices set corresponding to the assistant hyperplane. Inspired by this bisection idea, here we propose to use $\left(\boldsymbol{\theta}^{T} \mathbf{u}-p\right) / d$ and $\left(\boldsymbol{\theta}^{T} \mathbf{v}-p\right) / d$ as the lower bound and the upper bound of the difference coefficient, respectively. Our lower bound is tighter, and the calculation of the upper bound is simpler. In Fig. 3.6, we show the mean square error (MSE) of the projection result for the proposed projection algorithm and the proposed bisection projection algorithm for different values of input dimension and $I_{\max }$. It can be seen that for the same input dimension, the MSE of the bisection projection algorithm drops faster than that of the iterative projection algorithm. Specifically, 
the MSE of the bisection projection algorithm with 10 iterations is similar or less than that of the iterative projection algorithm with 40 iterations for input dimension (check node degree) larger than 9. With the increase of the input dimension, it can be clearly observed that the MSE becomes smaller for the same $I_{\max }$.

It should be noted that in the implementation of the bisection projection algorithm, we divide the input points into two classes as the CSA-based projection algorithm and the bisection framework is only activated for the case where $\Pi_{[0,]^{d}}(\mathbf{v}) \notin \mathbb{P P}_{d}$. The bisection projection algorithm is more appealing in terms of its fast convergence speed, while regarding the hardware implementation, the proposed iterative projection algorithm has an advantage.

\subsection{Conclusion}

In this chapter, we proposed an iterative check polytope projection algorithm to reduce the complexity of ADMM decoding of LDPC codes. The proposed projection algorithm converges with the increase of iterations. Contrary to the existing projection algorithms, the proposed algorithm avoids sorting operations and only involves simple arithmetic operations. The average complexity and the worst-case complexity of the proposed algorithm are both linear in the input dimension. This is in comparison with the complexity introduced by the sorting operation in the previous projection algorithms. Moreover, avoiding sorting operations enables efficient hardware implementation of ADMM decoding and boosts the potential for deploying the ADMM decoder in reality. Simulation results show that the proposed projection algorithm can achieve a similar FER performance with the accurate projection algorithm. Although the proposed projection algorithm is convergent, it may take more than 30 iterations for the projection algorithm to maintain the same FER performance. Therefore, a bisection framework is used to accelerate the convergence speed of the proposed algorithm. The upper bound and the lower bound of the difference coefficient are derived based on the same proposition used in the iterative projection algorithm. 


\section{Chapter 4}

\section{Reduced-Complexity Node-Wise Scheduling}

The ADMM decoding algorithm can be seen as an iterative message-passing algorithm performed over Tanner graphs [33]. For message-passing algorithms, the scheduling of messages plays an important in the convergence of the algorithm. The message scheduling schemes are intensively investigated for BP decoders. Therefore, it is possible to apply the scheduling schemes proposed for BP decoders on ADMM decoding. The layered scheduling, proposed in [53] for the BP decoder, was applied to ADMM decoding recently [33]. It is well known that the informed dynamic scheduling converges faster than the layered scheduling for BP decoding [57]. This motivates us to investigate the informed dynamic scheduling for ADMM decoders.

In this chapter, we first review the scheduling schemes for BP decoders, especially the informed dynamic scheduling schemes in Section 4.1. Then we apply the nodewise scheduling to the ADMM decoder in Section 4.2. We simplify the calculation of the message residual and propose a reduced-complexity node-wise scheduling scheme in Section 4.3. The computational complexities of the proposed algorithm and other algorithms are analyzed in Section 4.4. The simulation results are given in Section 4.5 .

\subsection{Scheduling Schemes for BP Decoders}

The flooding scheme updates all check node messages or all variable node messages in one iteration, which involves a significant number of unimportant updating operations. Apart from this, the newly updated check (variable) node messages cannot 
be used in the current iteration. In contrast, sequential scheduling schemes with a fixed order of message updating are shown to converge twice as fast as the flooding scheduling [52-54] for BP decoders. In these algorithms, the latest available messages can be used in the current iteration.

Later, the informed dynamic scheduling based on message residuals is investigated to further improve BP decoders' convergence rate [57]. In this scheme, the message updating order is determined by the dynamic change of messages, namely the message residual, throughout the decoding process. The message with the largest residual plays the most important role in making the algorithm converge and will be updated first.

Since then, a number of advanced dynamic selection strategies based on informed dynamic scheduling have been proposed [55,56,58-61]. If only the message with the maximum residual is updated, the scheduling scheme is called the edge-based residual scheduling, such as $[57,59]$. If all messages of the variable (check) node with the maximum residual are updated, the scheduling scheme is called the nodewise residual scheduling, such as [57]. The scheduling schemes can also be classified according to the types of the messages residuals used as the metric, namely the variable-to-check residuals in $[55,58,60,61]$ and the check-to-variable residuals in [57].

\subsection{Node-Wise Scheduling for ADMM Decoders}

We note that there are two differences between the ADMM the BP decoders. Firstly, for ADMM decoders, the messages sent from a variable node to different check nodes are the same, while for BP decoders, these messages are different. Secondly, the messages sent from a check node to different variable nodes are calculated simultaneously for ADMM decoders. These messages are calculated independently by BP decoders. The first difference implies that the scheduling based on variable-to-check residuals is not suitable for ADMM decoders. This is because all the neighboring check nodes of the variable node with maximum residual should be updated. In contrast, for the $\mathrm{BP}$ decoder, only one of the neighboring check nodes is considered. The updating of several check nodes increase the complexity burden for the ADMM decoder. The second difference suggests that we should use node-wise scheduling for the ADMM decoder instead of using the edge-based residuals. 
From the discussion, we propose the node-wise scheduling ADMM decoders (NSADMM) based on the check-to-variable message residual. The residual corresponding to each check node $c_{j}$ is defined as

$$
r\left(c_{j}\right)=\max _{i \in N_{c}(j)}\left|L_{j \rightarrow i}^{\text {new }}-L_{j \rightarrow i}^{\text {old }}\right|
$$

where $L_{j \rightarrow i}^{n e w}$ denotes the candidate message waiting to be propagated in the current iteration and $L_{j \rightarrow i}^{\text {old }}$ denotes the message that has been propagated in the last iteration. Let $\mathbf{L}_{j}$ denote the messages of $L_{j \rightarrow i}$ with $i \in N_{c}(j)$. The calculation of $r\left(c_{j}\right)$ at the $(k+1)$-th iteration is shown as follows:

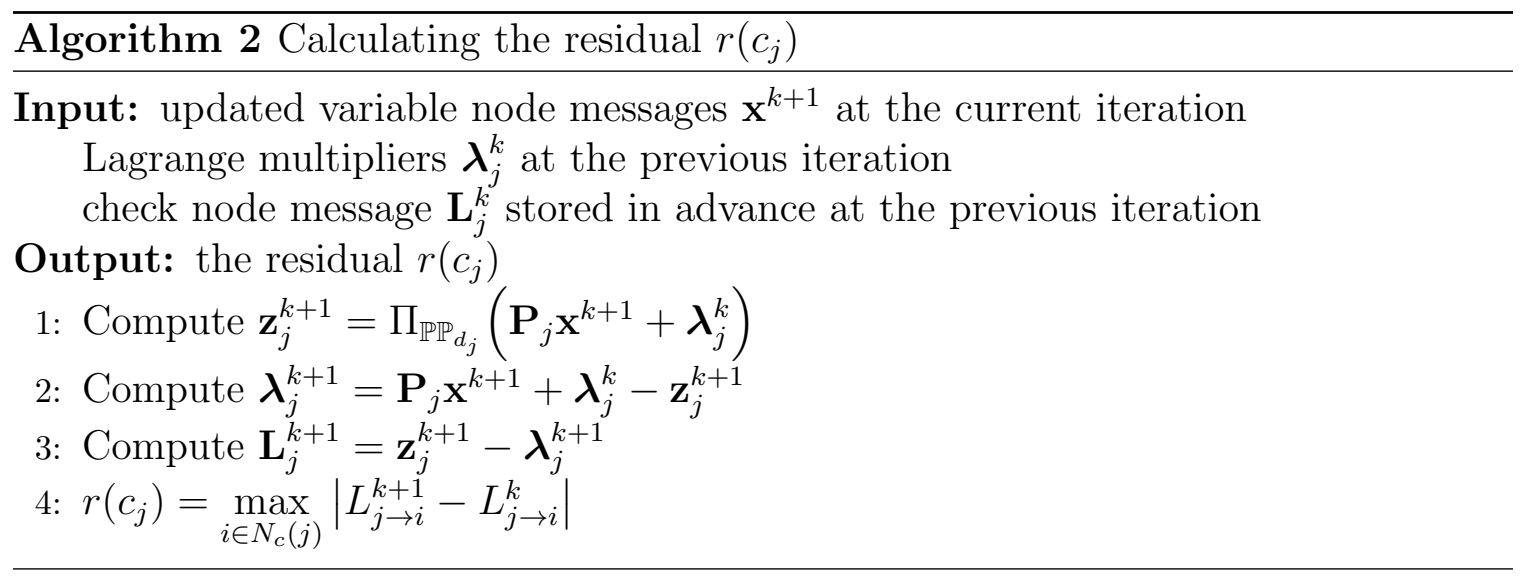

The scheduling steps of the NS-ADMM are described as follows. Firstly, the residuals corresponding to each check node are calculated. Suppose the maximum residual is attained at $c_{m}$, the following three steps need to be done: (1) check-tovariable message update: generate and propagate the message passed from check node $c_{m}$ to its neighboring variable nodes; (2) variable-to-check message update: for each neighboring variable node $v_{p}$ of $c_{m}$, generate and propagate the message passed from $v_{p}$ to its neighboring check nodes; (3) recalculate the corresponding residuals when the variable-to-check message is updated for all check nodes $c_{q}$ with $q \in N_{v}(p), \forall p \in N_{c}(m)$. The NS-ADMM decoding is formally described in Algorithm 3. The stopping rule can be similar to that in [33] or uses the early termination scheme based on the property of LDPC codes such as $\mathbf{H x}^{\mathrm{T}}=0$. 


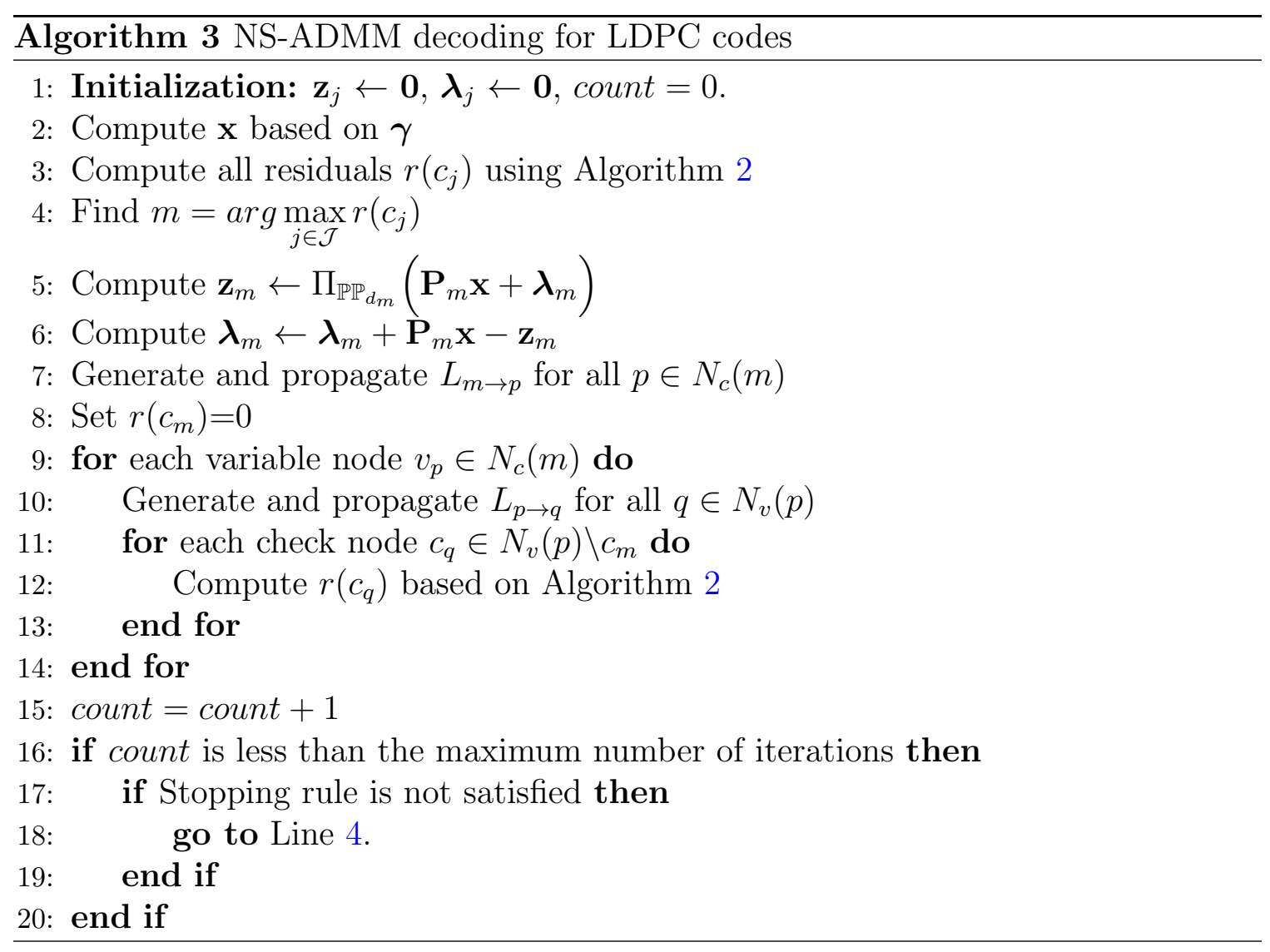

\subsection{Reduced-Complexity NS-ADMM}

To obtain residuals for the NS-ADMM decoding, we need to compute $\mathbf{z}_{j}$ by calling the check polytope projection which is a time-consuming operation in the ADMM decoder. To reduce the computational complexity, we propose a simple approximation method to calculate the residual. Suppose we are calculating the residual of check node $c_{j}$ at the $(k+1)$ th iteration. Let $\mathbf{w}_{j}^{k+1}=\mathbf{P}_{j} \mathbf{x}^{k+1}+\boldsymbol{\lambda}_{j}^{k}$. Then the difference between $\mathbf{L}_{j}^{k+1}$ and $\mathbf{L}_{j}^{k}$ can be calculated as follows:

$$
\begin{aligned}
\mathbf{L}_{j}^{k+1}-\mathbf{L}_{j}^{k} & =\left(\mathbf{z}_{j}^{k+1}-\boldsymbol{\lambda}_{j}^{k+1}\right)-\left(\mathbf{z}_{j}^{k}-\boldsymbol{\lambda}_{j}^{k}\right) \\
& =\left(\mathbf{z}_{j}^{k+1}-\mathbf{z}_{j}^{k}\right)-\left(\boldsymbol{\lambda}_{j}^{k+1}-\boldsymbol{\lambda}_{j}^{k}\right) \\
& =\left(\mathbf{z}_{j}^{k+1}-\mathbf{z}_{j}^{k}\right)-\left(\mathbf{w}_{j}^{k+1}-\mathbf{z}_{j}^{k+1}-\mathbf{w}_{j}^{k}+\mathbf{z}_{j}^{k}\right) \\
& =2\left(\mathbf{z}_{j}^{k+1}-\mathbf{z}_{j}^{k}\right)-\left(\mathbf{w}_{j}^{k+1}-\mathbf{w}_{j}^{k}\right) \\
& =2\left[\Pi_{\mathbb{P}_{d_{j}}}\left(\mathbf{w}_{j}^{k+1}\right)-\Pi_{\mathbb{P P}_{d_{j}}}\left(\mathbf{w}_{j}^{k}\right)\right]-\left(\mathbf{w}_{j}^{k+1}-\mathbf{w}_{j}^{k}\right) .
\end{aligned}
$$


As will be justified below, we now assume that the order of the point-wise difference between the given two vectors is preserved when the projection operation is applied to these two vectors separately. It means that in (4.2) the order of elements in $\mathbf{w}_{j}^{k+1}-\mathbf{w}_{j}^{k}$ is the same as the order of elements in $\prod_{\mathbb{P}_{d_{j}}}\left(\mathbf{w}_{j}^{k+1}\right)-\prod_{\mathbb{P}_{d_{j}}}\left(\mathbf{w}_{j}^{k}\right)$. Note that in the NS-ADMM algorithm we only need to find the maximum residual of the check nodes, and it is not necessary to calculate the exact value of residuals. Therefore, we can use $\mathbf{w}_{j}^{k+1}-\mathbf{w}_{j}^{k}$ to approximate $\prod_{\mathbb{P}_{d_{j}}}\left(\mathbf{w}_{j}^{k+1}\right)-\prod_{\mathbb{P P}_{d_{j}}}\left(\mathbf{w}_{j}^{k}\right)$ in (4.2). Then we have

$$
\mathbf{L}_{j}^{k+1}-\mathbf{L}_{j}^{k} \approx 2\left(\mathbf{w}_{j}^{k+1}-\mathbf{w}_{j}^{k}\right)-\left(\mathbf{w}_{j}^{k+1}-\mathbf{w}_{j}^{k}\right)=\mathbf{w}_{j}^{k+1}-\mathbf{w}_{j}^{k} .
$$

Finally, the residual $r\left(c_{j}\right)$ can be obtained as the largest absolute value of the components in $\mathbf{w}_{j}^{k+1}-\mathbf{w}_{j}^{k}$. By inspecting Algorithm 3, the residual calculation can be simplified further as follows. When computing the residual $r\left(c_{q}\right)$ in Line 12 of Algorithm 3 based on (4.3), all the variable-to-check messages $L_{v \rightarrow q}, v \in N_{c}(q)$ are unchanged except for $L_{p \rightarrow q}$ if the Tanner graph does not have 4-cycles. This implies that only one component in $\mathbf{P}_{q} \mathbf{x}$ is changed. Thus $\mathbf{w}_{q}^{k+1}-\mathbf{w}_{q}^{k}$ has only one nonzero component which is associated with $v_{p}$. The absolute value of this non-zero component is the residual. In a word, the residual calculation based on (4.3) using vector subtraction followed by a maximum operation is reduced by simple scalar calculations. The ADMM decoder with the reduced-complexity node-wise scheduling is denoted by RC-NS-ADMM.

The assumption we used to derive (4.3) does not strictly hold. To see this, we define an event $\Psi$ to represent the event that the index of the maximum element in $\prod_{\mathbb{P}_{d_{j}}}\left(\mathbf{w}_{j}^{k+1}\right)-\prod_{\mathbb{P}_{d_{j}}}\left(\mathbf{w}_{j}^{k}\right)$ is equal to the index of the maximum element in $\mathbf{w}_{j}^{k+1}-\mathbf{w}_{j}^{k}$. Then we use the Monte Carlo simulation to estimate the probability $\operatorname{Pr}(\Psi)$ for the NS-ADMM decoding. Fig. 4.1 shows the estimated values of $\operatorname{Pr}(\Psi)$ for four LDPC codes at different $E_{b} / N_{0}$ values over the AWGN channel. It can be seen that the estimated values of $\operatorname{Pr}(\Psi)$ for all the simulated LDPC codes are close to $90 \%$ as $E_{b} / N_{0}$ increases. From the perspective of selecting the maximum residual in the NS-ADMM, the results in Fig. 4.1 implies that the proposed method becomes more reliable to approximate the residual calculation as $E_{b} / N_{0}$ increases. 


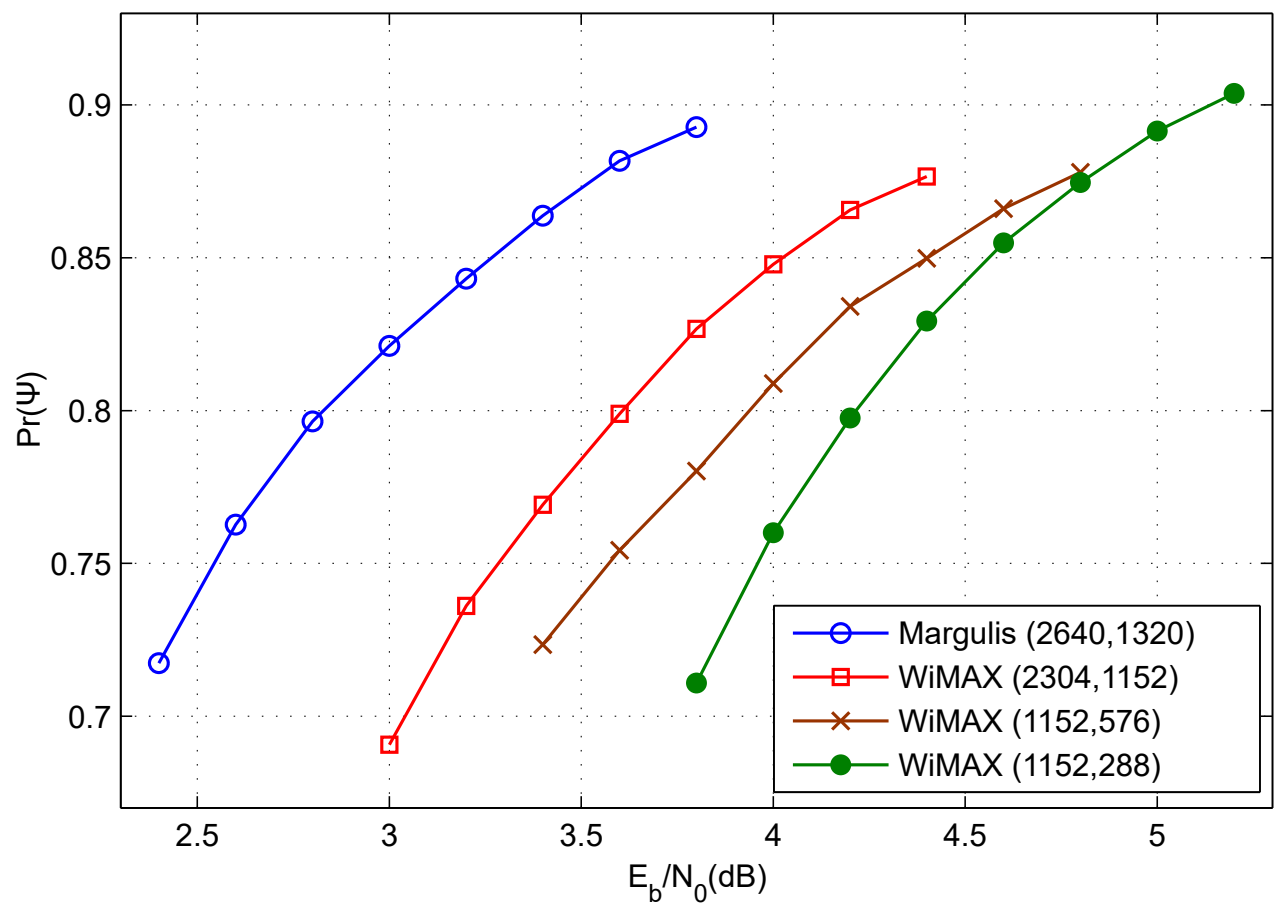

Figure 4.1: Empirical results for the estimated values of $\operatorname{Pr}(\Psi)$ for four LDPC codes at different $E_{b} / N_{0}$ values over the AWGN channel.

\subsection{Computational Complexity Analysis}

For NS-ADMM and RC-NS-ADMM decoding of an LDPC code with $n$ variable nodes and $m$ check nodes, one iteration includes $m$ executions of the procedure Lines 4 14 in Algorithm 3. The complexity of the proposed method is evaluated by two parts: the message updates and the residual calculations. The message updates are analyzed by evaluating the number of updating operations for $\mathbf{x}, \mathbf{z}$ and $\boldsymbol{\lambda}$. The residual calculations are analyzed by evaluating the number of addition/subtractions (noted additions) and the number of projections. Let the average degree of variable and check nodes be $d_{v}$ and $d_{c}$, respectively. Table 4.1 shows the number of message updates and residual calculations per iteration for four scheduling schemes. The number of message updates for the four schemes is clear. It should be noted that one update of $x$ involves $d_{v}+1$ additions, 1 multiplication and 1 projection onto interval $[0,1]$ based on (2.24) when the LP model is used. One update of $\mathbf{z}$ involves $d_{c}$ additions and 1 polytope projection. One update of $\boldsymbol{\lambda}$ involves $2 d_{c}$ additions. For the residual calculations, one execution of the procedure Lines 4-14 in Algorithm 3 needs $d_{v}\left(d_{c}-1\right)$ residual calculations. For the NS-ADMM, one residual calculation needs one check 
Table 4.1: Complexity comparison of message updates and residual calculations per iteration for different scheduling schemes

\begin{tabular}{|c|c|c|c|c|c|c|}
\hline \multirow{2}{*}{ Scheduling schemes } & \multicolumn{3}{|c|}{ for message updates } & \multicolumn{3}{c|}{ for residual calculations } \\
\cline { 2 - 7 } & $x_{i}$ updates & $\mathbf{z}$ updates & $\boldsymbol{\lambda}$ updates & comparisons & additions & projections \\
\hline flooding-ADMM & $n$ & $m$ & $m$ & 0 & 0 & 0 \\
\hline layered-ADMM & $m d_{c}$ & $m$ & $m$ & 0 & 0 & 0 \\
\hline NS-ADMM & $m d_{c}$ & $m$ & $m$ & $m-1+d_{v}\left(d_{c}-1\right)^{2}$ & $4 m d_{v} d_{c}\left(d_{c}-1\right)$ & $m d_{v}\left(d_{c}-1\right)$ \\
\hline RC-NS-ADMM & $m d_{c}$ & $m$ & $m$ & $m-1$ & $2 m d_{v}\left(d_{c}-1\right)$ & 0 \\
\hline
\end{tabular}

polytope projection and four vector additions with length $d_{c}$. For the RC-NS-ADMM, one residual calculation needs two additions. For comparisons, Line 4 in Algorithm 3 needs $m-1$ comparisons. For the NS-ADMM, each residual calculation needs $d_{c}-1$ comparisons. While for the RC-NS-ADMM, the residual calculation does not need comparisons. It should be noted that the check polytope projection operation also involves some basic arithmetic operations based on the implementation.

From Table 4.1 we mainly want to show that the RC-NS-ADMM saves all check polytope projections and a large number of additions used in the NS-ADMM. Compared to the layered-ADMM, the RC-NS-ADMM needs extra comparisons and additions for residual calculation, but the number of extra operations is at a reasonable level. For example, the total number of additions implicitly involved in the updates of $x_{i}, \mathbf{z}$ and $\boldsymbol{\lambda}$ is $m d_{c}\left(d_{v}+4\right)$. Furthermore, the RC-NS-ADMM converges several times faster than the layered-ADMM as shown in the next section. This will reduce the total number of operations in the whole iterations.

\subsection{Simulation Results}

We use the $(576,288)$ rate- 0.5 code $\mathcal{C}_{1}$ from the IEEE 802.16 e standard. To accelerate simulations, all the ADMM decoders use over-relaxation and early termination. The over-relaxation parameter is set to 1.9 [33]. The ADMM decoder used here is actually the ADMM VN-penalized decoder with $\ell_{1}$ penalty terms [66]. The Lagrangian parameter $\mu$ and the penalty coefficient $\alpha$ are optimized, and we choose $\mu=4.0$ and $\alpha=0.8$.

Fig. 4.2 shows the FER performances of $\mathcal{C}_{1}$ versus the number of iterations for ADMM decoders with different scheduling schemes. It can be seen that the convergence behavior of RC-NS-ADMM is similar to that of NS-ADMM, although the 


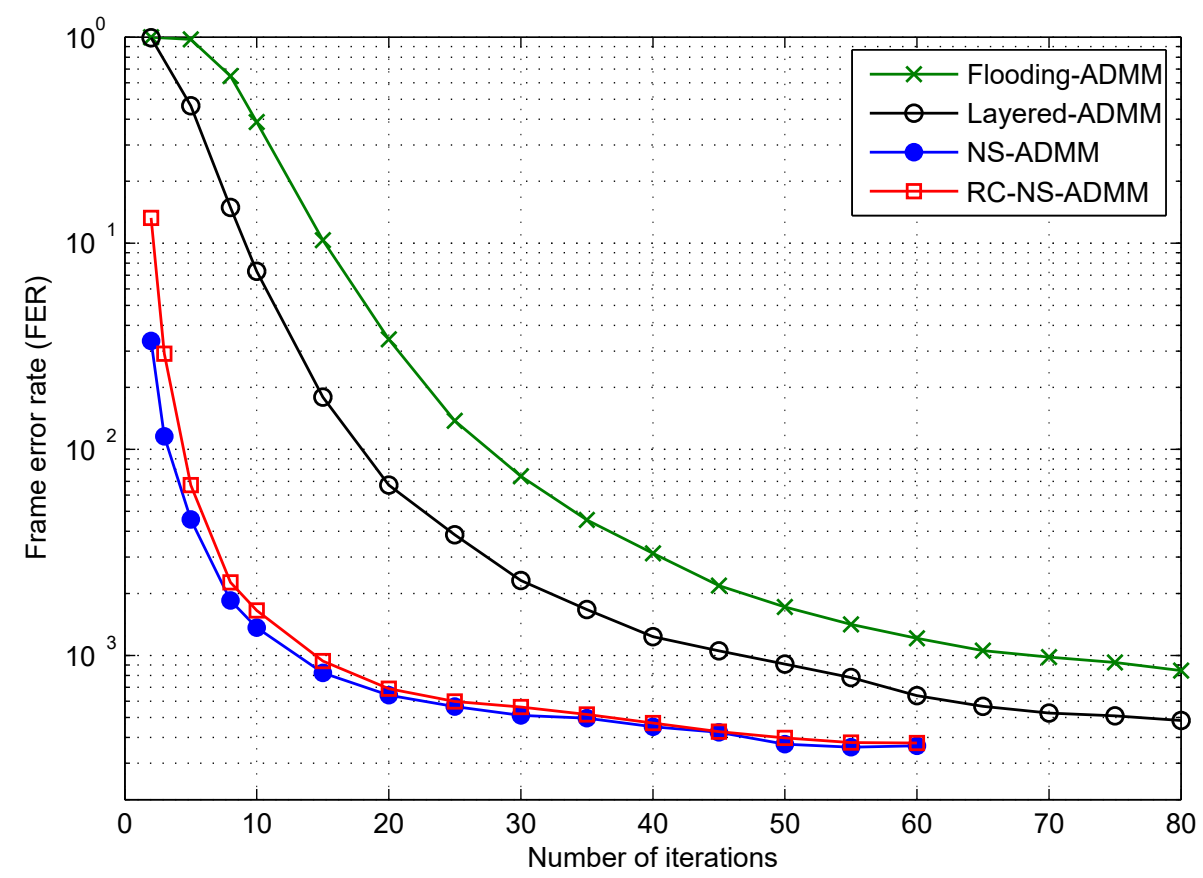

Figure 4.2: FER performance of $\mathcal{C}_{1}$ vs. number of iterations under ADMM decoders with various scheduling schemes at $E_{b} / N_{0}=2.8 \mathrm{~dB}$.

inaccurate calculation of the check node message residual is used. The convergence rate for the RC-NS-ADMM is much faster than that for the flooding and the layered scheduling. The reason behind this is that the informed dynamic scheduling strategy used in the RC-NS-ADMM focuses on the part of the Tanner graph that has not converged [57].

Fig. 4.3 shows FER performances of ADMM decoder with various scheduling schemes when the maximum number of iterations is set to 20 . It can be seen that the performance gap between NS-ADMM and flooding-ADMM or layered-ADMM increases with the value of $E_{b} / N_{0}$. This is because, in general, the ADMM algorithm requires enough iterations to converge [34]. At low $E_{b} / N_{0}$ values, the error frames can not be corrected by all the decoders within 20 iterations. While at high $E_{b} / N_{0}$ values, some frames that can not be corrected by the flooding-ADMM or layered-ADMM within 20 iterations can be corrected by the NS-ADMM due to its faster convergence rates. The FER of the proposed NS-ADMM is improved by more than two orders of magnitude at $4.0 \mathrm{~dB}$ compared with the flooding-ADMM scheme. Moreover, the performances of NS-ADMM and RC-NS-ADMM are closer at high $E_{b} / N_{0}$ values. This can be explained by Fig. 4.1 where the probability of the two decoders selecting 


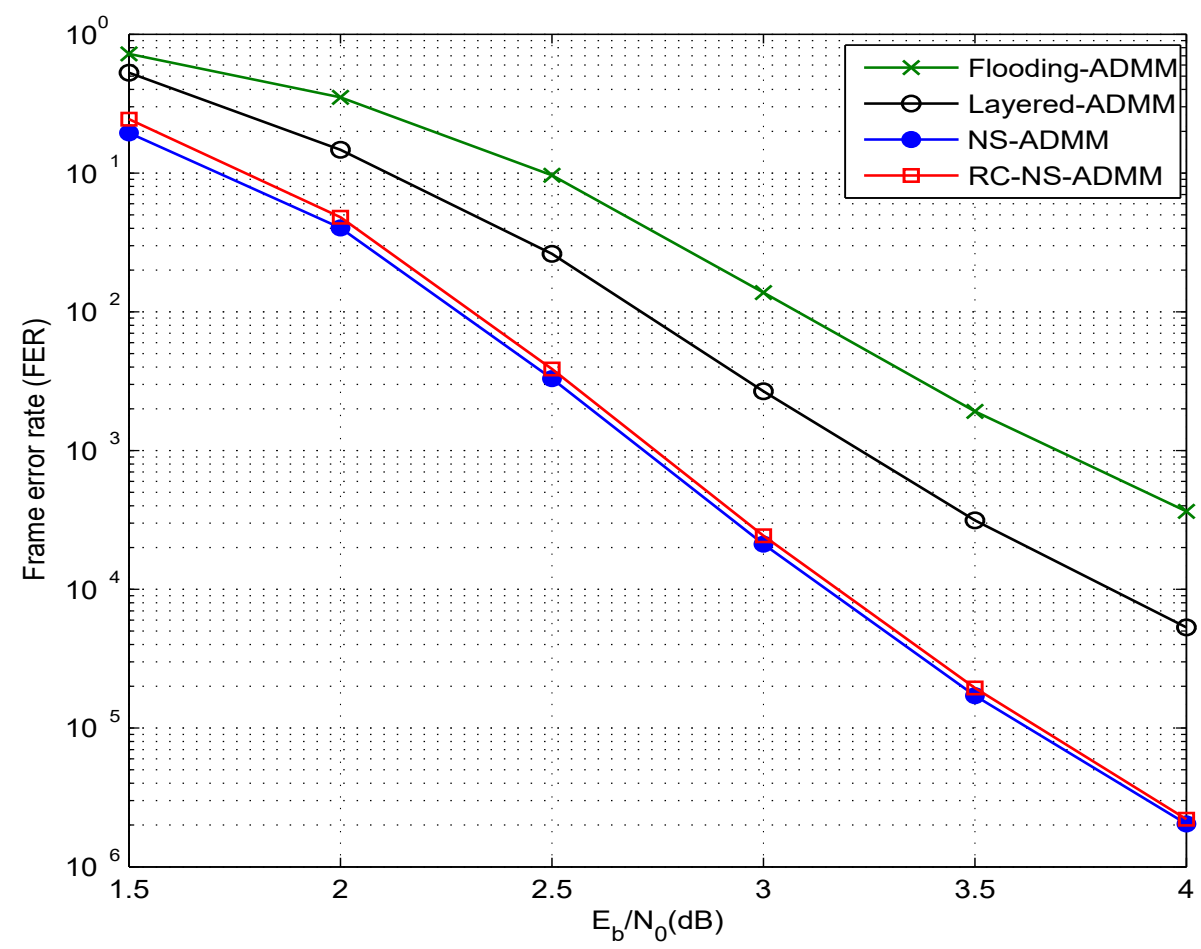

Figure 4.3: FER performance of $\mathcal{C}_{1}$ vs. $E_{b} / N_{0}$ under $\mathrm{ADMM}$ decoders with various scheduling schemes. The maximum number of iterations is set to 20 .

the same update nodes increases as $E_{b} / N_{0}$ increases.

We also compare the average number of iterations for different schemes in Fig. 4.4. It can be seen that the average number of iterations for RC-NS-ADMM is about 2.3 times less than the layered-ADMM and 3.5 times less than the flooding$\mathrm{ADMM}$ as $E_{b} / N_{0}$ increases. In particular, when $E_{b} / N_{0}=4.0 \mathrm{~dB}$, the average number of iterations is 2.0 for the RC-NS-ADMM, while it is 4.5 for the layered-ADMM. Combined with the complexity analysis in Section 4.4, it means that the RC-NSADMM saves $2.5 m d_{c}$ updating operations for $\mathbf{x}, 2.5 m$ updating operations for $\mathbf{z}$ and $2.5 m$ updating operations for $\boldsymbol{\lambda}$ at the cost of $4 m d_{v}\left(d_{c}-1\right)$ additions and $2(m-1)$ comparisons. Compared with the complexity of the check polytope projection in the updating operation of $\mathbf{z}$, the extra computation of residuals for the RC-NS-ADMM is acceptable. 


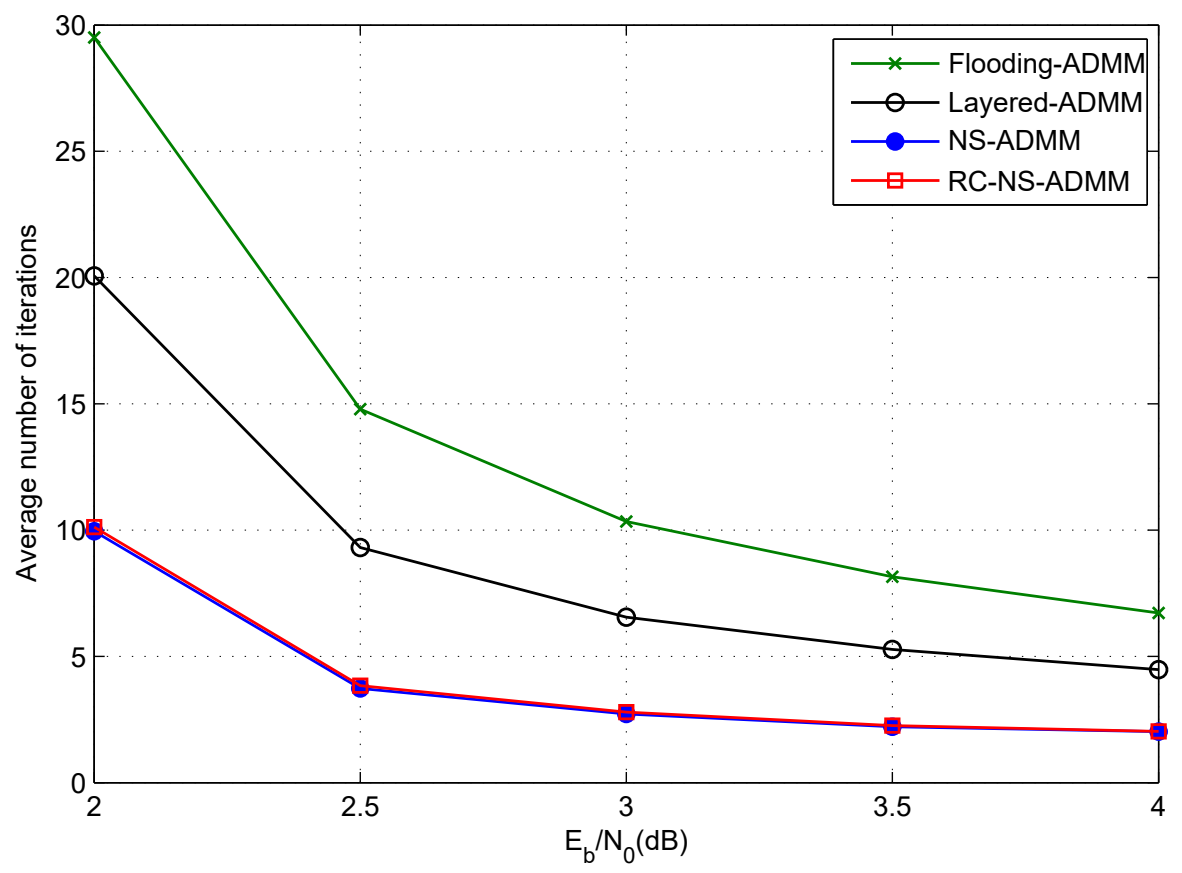

Figure 4.4: Average number of iterations of $\mathcal{C}_{1}$ under ADMM decoders with various scheduling schemes at different $E_{b} / N_{0}$.

\subsection{Conclusion}

In this chapter, we study the node-wise scheduling of the ADMM decoder and propose a reduced-complexity node-wise scheduling scheme, i.e., RC-NS-ADMM, for ADMM decoding of LDPC codes. Simulations show that the RC-NS-ADMM performs similarly to the NS-ADMM in terms of convergence rate and FER performance. Meanwhile, the proposed method converges significantly faster than flooding scheduling and layered scheduling. 


\section{Chapter 5}

\section{ADMM CN-Penalized Decoder}

Although preserving advantages over BP decoders, the error correction performance of the ADMM decoder is not comparable to BP decoders, especially in the low SNR region. In order to improve the error correction performance of ADMM decoding at low SNR values, penalty terms were added to the objective function of the LP decoding model to penalize the pseudo-codewords. All previous ADMM penalized decoding algorithms [66-68] penalize the invalid solutions from the aspect of the variable nodes and are referred to ADMM VN-penalized decoders.

In this chapter, we first review the ADMM VN-penalized decoder in Section 5.1. Then, in Section 5.2, we propose the ADMM CN-penalized decoder, whose penalty terms are related to the check nodes. Descriptions of functions that can be used as the $\mathrm{CN}$-penalty terms are present, and several examples are given. The convergence conclusion and the all-zero assumption of the ADMM CN-penalized decoding algorithm are proved. We analyze the computational complexities of the proposed algorithm with different penalty functions and other algorithms in Section 5.3. The instanton analysis method is introduced in Section 5.4. Simulation results are given in Section 5.5 .

\subsection{ADMM VN-Penalized Decoder}

Simulation results show that the ADMM decoder suffers poor FER performance in the low SNR region. It produces non-integral solutions (pseudo-codewords) when it fails to recover the transmitted codeword. Since the ADMM decoder always finds solutions in the feasible region with the minimum objective function value, we know that the pseudo-codeword has a smaller objective function value than the codewords. 
Thus, in [66], the authors introduced an ADMM penalized decoding model to suppress the pseudo-codewords. Penalty functions with respect to variable nodes are added to the objective function of the ADMM decoding model as follows:

$$
\begin{array}{rll}
\operatorname{minimize} & \boldsymbol{\gamma}^{T} \mathbf{x}+\sum_{i}^{n} h\left(x_{i}\right) & \\
\text { subject to } & \mathbf{P}_{j} \mathbf{x}=\mathbf{z}_{j}, & \forall j \in \mathcal{J}, \\
& \mathbf{z}_{j} \in \mathbb{P P}_{d_{j}}, & \forall j \in \mathcal{J},
\end{array}
$$

where $h\left(x_{i}\right)$ is the penalty function with respect to $x_{i}$. The penalty function is an increasing function on $[0,0.5)$ and symmetric about 0.5 . For $x_{i}$ closer to 0.5 , the penalty function value is larger. This ensures that solutions closer to point $\mathbf{0 . 5}$ have a larger penalty than solutions with all binary components. Due to the extra cost of penalty functions, fractional solutions are penalized and integer solutions are preferred.

It should be noted that the objective function of (5.1) is not linear anymore. Therefore, the convergence guarantee derived from the LP model does not hold anymore. However, the ADMM technique can still be applied to solve (5.1) and obtain a local minimum efficiently. Moreover, this model still preserves the important ML Certificate property.

To solve (5.1), we first need to obtain its augmented Lagrangian. The updating rules of $f(\mathbf{x}), \mathbf{z}_{j}$ and $\boldsymbol{\lambda}_{j}$ can be obtained by solving (2.16), (2.17) and (2.18). It can be verified that for different objective functions in (5.1), the updating rules of $\mathbf{z}_{j}$ and $\boldsymbol{\lambda}_{j}$ do not change. The change of the objective function only impacts the updating rule of $x_{i}$. Generally, for any objective function $f(\mathbf{x})$, the update of $x_{i}$ in the $(k+1)$-th iteration is the root of the following equation:

$$
x_{i}=\frac{1}{d_{i}}\left(\sum_{j \in N_{v}(i)}\left(z_{j(i)}^{k}-\lambda_{j(i)}^{k}\right)-\frac{1}{\mu} \frac{\partial f\left(\mathbf{x}^{k}\right)}{\partial x_{i}}\right),
$$

in which $x_{i}$ is the unknown variable, and $\frac{\partial f\left(\mathbf{x}^{k}\right)}{\partial x_{i}}$ is the partial derivative of $f(\mathbf{x})$ with respect to $x_{i}$ calculated with the elements of $\mathbf{x}$ (except for $x_{i}$ ) in the $k$-th iteration. Then the calculated value of $x_{i}$ is projected into the interval $[0,1]$. For the case where multiple roots for (5.2) exist, the farthest root from 0.5 can be used because binary values are preferred. Interested readers are encouraged to refer to [34] and [66].

The $\ell_{1}, \ell_{2}$ and $\log$ penalty functions were proposed in [66], namely $h_{1}(x)=$ 
$-\alpha|x-0.5|, h_{2}(x)=-\alpha(x-0.5)^{2}$ and $h_{3}(x)=-\alpha \log (|x-0.5|)$, where $\alpha$ is called the penalty coefficient and is determined in advance by simulations. Experiments show that decoding with the $h_{2}(x)$ penalty function achieves a relatively better error correction performance, and its average decoding time is also less than the other two. Its updating rule for $x_{i}$ is

$$
x_{i}^{k+1}=\Pi_{[0,1]} \frac{1}{d_{i}-\frac{2 \alpha}{\mu}}\left(\sum_{j \in N_{v}(i)}\left(z_{j(i)}^{k}-\lambda_{j(i)}^{k}\right)-\frac{\gamma_{i}}{\mu}-\frac{\alpha}{\mu}\right) .
$$

Later, Wang et al. proposed another penalty function which is a generalization of $h_{1}(x)$ [68]. The penalty function is segmented, and each segment is in the form of $-\alpha_{t}|x-0.5|$, but with a different penalty coefficient. The segment boundaries and the penalty coefficients for different segments are optimized by differential evolution. As a result, $x_{i}$ in different segments will be penalized by a different function. The penalty functions introduced in [66] and [68] are all based on variable nodes. We thus term them as VN-penalty functions and refer to the decoding algorithms as ADMM VN-penalized decoding algorithms.

The all-zero assumption holds for ADMM VN-penalized decoding algorithms. It means the failure probability of the decoding algorithm is independent of the transmitted codewords for symmetric channels. A revised proof of the all-zero assumption, Theorem 6 of [66], is given in Appendix A.

\subsection{ADMM CN-penalized Decoder}

In this section, we propose a novel ADMM CN-penalized decoding model. The properties of the penalty functions that can be used as CN-penalty terms are given. The convergence of the ADMM CN-penalized decoding algorithm and the validation of the all-zero assumption are proved under these requirements. Several examples of the CN-penalty functions and their updating rules are presented.

\subsubsection{ADMM CN-Penalized Decoding Model}

When the ADMM decoder fails to recover the transmitted codeword, the non-integer components of pseudo-codewords are not the only characteristics that can be used to increase the value of the objective function. We notice that some parity checks of the 
hard-decision values of the solution are non-zero. The proposed algorithm penalizes the solutions which cannot satisfy all the parity-checks, so that the codeword will have a larger chance of being selected. We add penalty terms to the objective function of the ADMM decoding model as follows:

$$
\begin{array}{rlr}
\operatorname{minimize} & \boldsymbol{\gamma}^{T} \mathbf{x}+\alpha \sum_{j=1}^{m} g\left(\mathbf{P}_{j} \mathbf{x}\right) & \\
\text { subject to } & \mathbf{P}_{j} \mathbf{x}=\mathbf{z}_{j}, & \forall j \in \mathcal{J}, \\
& \mathbf{z}_{j} \in \mathbb{P P}_{d_{j}}, & \forall j \in \mathcal{J} .
\end{array}
$$

Inside (5.4), $g\left(\mathbf{P}_{j} \mathbf{x}\right)$ is the penalty function corresponding to check node $j$ and $\alpha$ is the penalty coefficient. The penalty function takes variables corresponding to the neighboring variable nodes of check node $j$ as input. Here we do not use the idea in [67] which assigns different penalty coefficients to check nodes with different degrees, because the degree distribution of check nodes is usually not as rich as variable nodes. The impact of using different penalty coefficients is rather small. In order to be used as penalty terms, the CN-penalty function $g(\mathbf{x})$ should have the following properties:

1. $g(\mathbf{x})$ should be able to take different number of arguments as input ${ }^{1}$;

2. $g(\mathbf{x})=g(\mathbf{y})$, if $\mathbf{x}$ is a permutation of $\mathbf{y}$;

3. $g(\mathbf{x})=g(\mathbf{y})$, if $\mathbf{x}$ is a subvector of $\mathbf{y}$ and the other elements of $\mathbf{y}$ are zeros;

4. $g(\mathbf{x})$ should be a monotonic function of each element of $\mathbf{x}$ over $[0,1]$;

5. Consider two vectors $\mathbf{x}$ and $\mathbf{y}$, where an odd number of elements of $\mathbf{x}$ and $\mathbf{y}$ satisfy $y_{i}=1-x_{i}$, and the rest of their elements satisfy $y_{i}=x_{i}$. Then, $g(\mathbf{x})+g(\mathbf{y})=a+b$, where $a=g(0,0)$ and $b=g(0,1) ;$

6. $g(\mathbf{x})$ is partially differentiable for each element in the interval $[0,1]$.

For irregular LDPC codes, the check node degree is not unique. The CN-penalty functions should possess Property 1 to be able to take vectors of different dimensions as input. Then the CN-penalty function can be used to penalize check nodes with different degrees. Property 2 guarantees that the value of the penalty function will not

\footnotetext{
${ }^{1}$ In this thesis, we do not discuss the case where the number of arguments is equal to 1.
} 
be affected by the arguments' order. We also note here functions with this property are so-called symmetric functions. Property 3 indicates that taking more 0 s as the parameters of the CN-penalty function does not affect the value of the function. It will be shown later that adding an even number of ones to the function input also does not affect the value of the function. Property 4 only requires the function to be monotonic with respect to each single variable. So for different variables, the function can be monotonically increasing or decreasing. This property encourages solutions that are close to 0 or 1 values. Property 5 defines $a$ and $b$ and the only requirement on them is $a \neq b$. It means $a$ and $b$ can be any real numbers. Property 5 can be used to derive the following important corollaries combined with the other properties.

Corollary 1. For a function $g(\mathbf{x})$ satisfying Properties 1 to 5 , and two vector $\mathbf{x}$ and $\mathbf{y}$ of the same length, if there is an even number of elements satisfying $y_{i}=1-x_{i}$, and the rest of the elements satisfy $y_{i}=x_{i}$, then $g(\mathbf{x})=g(\mathbf{y})$.

Proof. Consider vectors $\mathbf{x}$ and $\mathbf{y}$ described in the corollary, and define a vector $\mathbf{z}$ from $\mathbf{x}$ by changing an arbitrary element of $\mathbf{x}$, say $x_{i}$, to $1-x_{i}$. By the construction of $\mathbf{z}$, there is only one element of $\mathbf{z}$, i.e., $z_{i}=1-x_{i}$, that differs from the elements of $\mathbf{x}$. By Property 5, we thus have $g(\mathbf{x})+g(\mathbf{z})=a+b$. It can also be seen that there are an odd number of elements of $\mathbf{y}$ and $\mathbf{z}$ that satisfy $y_{i}=1-z_{i}$, and the rest of their elements are the same. Again, by Property 5, $g(\mathbf{y})+g(\mathbf{z})=a+b$. From $g(\mathbf{x})+g(\mathbf{z})=g(\mathbf{y})+g(\mathbf{z})=a+b$, we conclude that $g(\mathbf{x})=g(\mathbf{y})$.

Corollary 2. For a function $g(\mathbf{x})$ satisfying Properties 1 to 5 , all binary vectors with even weights have the same function value $a=g(0,0)$, and all binary vectors with odd weights have the same function value $b=g(0,1)$.

Proof. We consider two arbitrary binary vectors $\mathbf{x}$ and $\mathbf{y}$, both with even (or odd) weights and show that the function value is the same for both of them, and is thus equal to $g(0,0)$ (or $g(0,1))$. First, we consider a scenario where both vectors have the same length. In this scenario, it is easy to see that regardless of whether the vectors have even or odd weights, the condition of Corollary 1 is satisfied, i.e., an even number of elements of $\mathbf{x}$ and $\mathbf{y}$ satisfy $x_{i}=1-y_{i}$, and the rest satisfy $x_{i}=y_{i}$. From Corollary 1, we thus have $g(\mathbf{x})=g(\mathbf{y})$. For the second scenario where the two vectors have different lengths, the shorter vector can be appended with zeros to make the lengths equal. By Property 3 , this does not change the value of the function. The proof for this scenario will then be the same as that of the first scenario. 
Corollary 3. For a function $g(\mathbf{x})$ satisfying Properties 1 to 5 , the function values of a vector with elements in $[0,1]$ is in the range $[\min (a, b), \max (a, b)]$.

Proof. Suppose $\mathbf{x}=\left(x_{1}, x_{2}, x_{3}, \ldots, x_{n}\right)$ is a vector with $0 \leq x_{i} \leq 1$, its function value is bounded by $g\left(0, x_{2}, x_{3}, \ldots, x_{n}\right)$ and $g\left(1, x_{2}, x_{3}, \ldots, x_{n}\right)$ based on Property 4 . The smaller value is the lower bound, and the larger value is the upper bound. We also know $g\left(0, x_{2}, x_{3}, \ldots, x_{n}\right)$ is bounded by $g\left(0,0, x_{3}, \ldots, x_{n}\right)$ and $g\left(0,1, x_{3}, \ldots, x_{n}\right)$. So, by recursion, $g(\mathbf{x})$ is bounded by its values for two binary vectors. There are only two possible function values for binary vectors, $a$ and $b$, which are the function values of the even weight binary vector and the odd weight binary vector, respectively. This completes the proof.

As noted before, when the objective function of the LP model is changed, the updating rule of $x_{i}$ changes while the updating rules of $\mathbf{z}_{j}$ and $\boldsymbol{\lambda}_{j}$ remain the same. In order to get the updating rules of $x_{i}$ in (5.4), we need to find the partial derivative of $g\left(\mathbf{P}_{j} \mathbf{x}\right)$ with respect to $x_{i}$. If $x_{i} \notin N_{j}, \frac{\partial g\left(\mathbf{P}_{j} \mathbf{x}\right)}{\partial x_{i}}=0$ since variable node $i$ is not a neighbor of check node $j$ and $x_{i}$ is not involved in $\mathbf{P}_{j} \mathbf{x}$. The updated value of $x_{i}$ in this model is the root of the following equation

$$
x_{i}=\frac{1}{d_{i}}\left(\sum_{j \in N_{v}(i)}\left(z_{j(i)}^{k}-\lambda_{j(i)}^{k}-\frac{\alpha}{\mu} \frac{\partial g\left(\mathbf{P}_{j} \mathbf{x}^{k}\right)}{\partial x_{i}}\right)-\frac{\gamma_{i}}{\mu}\right),
$$

and then should be projected to the interval $[0,1]$.

Like [39], ADMM CN-penalized decoding can be expressed in the form of an iterative message-passing algorithm, as described in Algorithm 4. Inside the algorithm, we first initialize $\mathbf{x}$ with the hard decision values of the channel outputs and then start the iteration. The updating of $\mathbf{z}_{j}$ and $\boldsymbol{\lambda}_{j}$ can be considered as the updating of a check node. For ADMM-based LP decoding, each check node receives its neighboring variable node values and computes its outgoing messages $E_{j \rightarrow i}^{k+1}=z_{j(i)}^{k+1}-\lambda_{j(i)}^{k+1}$. For ADMM CN-penalized (or VN-penalized) decoding, these outgoing messages are adjusted by adding the penalty information before they are passed to the neighboring variable nodes. For each variable node, its value is updated based on the messages from its neighboring check nodes and the channel output. The termination condition of the algorithm is to reach the maximum number of iterations, and the output is the value of $\mathbf{x}$ in the last iteration. The early termination (lines 15-18) can be optionally enabled, and the output would be the hard-decision of $\mathbf{x}$ if all parity-checks are 
satisfied by the hard-decision of $\mathbf{x}$. The normally used termination condition which requires the largest difference between $\mathbf{P}_{j} \mathbf{x}^{k+1}$ and $\mathbf{z}_{j}^{k+1}$ less than a threshold is not used here. This is because the computation introduces additional complexity, and there is no guarantee of finding the global minimum even if this condition is satisfied. Instead, we give the following convergence result.

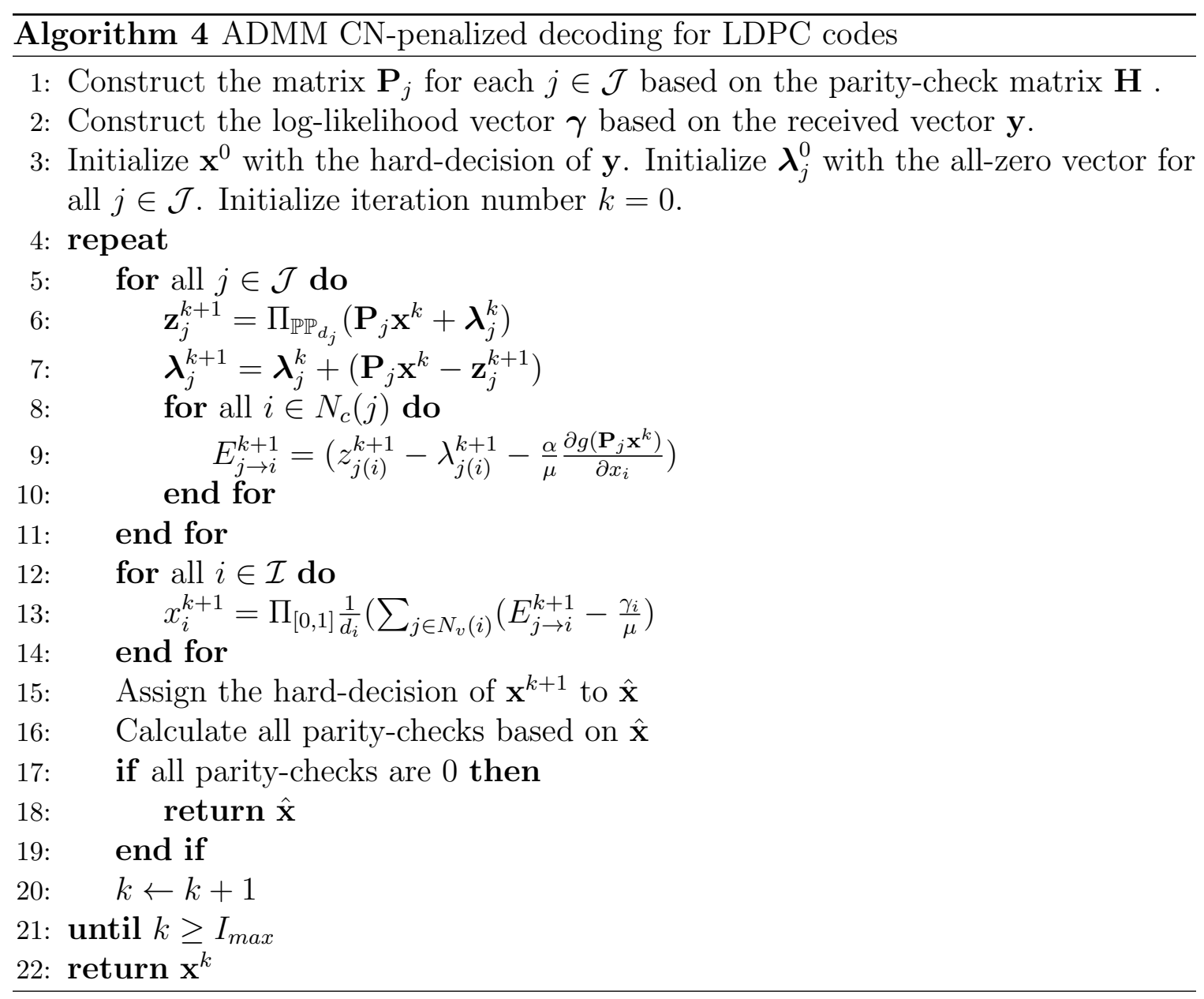

Proposition 7. If the output of Algorithm 4 is integer, then it is a valid codeword.

Proof. For the implementation without considering early termination, this is true because all integer solutions in the feasible region are valid codewords. For the implementation with early termination enabled, some binary solution would be output in advance if all parity-checks are satisfied. It means the binary output is a valid codeword. 
Proposition 8. If the output of Algorithm 4 is a global minimum of (5.4) and is also integer, then it is the $M L$ solution.

Proof. Suppose the global minimum is $\mathbf{x}^{*}$. For any other codeword $\mathbf{c}$, we have

$$
\boldsymbol{\gamma}^{T} \mathbf{x}^{*}+\sum_{j=1}^{m} \alpha g\left(\mathbf{P}_{j} \mathbf{x}^{*}\right) \leq \boldsymbol{\gamma}^{T} \mathbf{c}+\sum_{j=1}^{m} \alpha g\left(\mathbf{P}_{j} \mathbf{c}\right)
$$

From Proposition 7, we know $\mathbf{x}^{*}$ is also a codeword. Then $\mathbf{P}_{j} \mathbf{x}^{*}$ and $\mathbf{P}_{j} \mathbf{c}$ are all binary vectors containing an even number of 1 s for all $j \in \mathcal{J}$. Corollary 2 shows the values of penalty terms $g\left(\mathbf{P}_{j} \mathbf{x}^{*}\right)$ and $g\left(\mathbf{P}_{j} \mathbf{c}\right)$ are the same. Therefore, we obtain $\gamma^{T} \mathbf{x}^{*} \leq \boldsymbol{\gamma}^{T} \mathbf{c}$, which means $\mathbf{x}^{*}$ is an ML solution.

Theorem 2. If the channel is symmetric, then the probability that Algorithm 4 fails to find the transmitted codeword is independent of the codeword that was transmitted.

Proof. See Appendix B.

\subsubsection{Possible Penalty Functions}

Three functions that can be used as the penalty function are given here.

1)

$$
g_{1}\left(x_{1}, x_{2}, \ldots, x_{n}\right)= \begin{cases}g_{1}\left(g_{1}\left(x_{1}, x_{2}, \ldots, x_{n-1}\right), x_{n}\right), & \text { if } n>2 \\ x_{1}+x_{2}-2 x_{1} x_{2}, & \text { if } n=2 .\end{cases}
$$

This function is described in a recursive way. It can be shown that it satisfies all the requirements to be used as $\mathrm{CN}$-penalty functions. If $n=2$, this function is equivalent to the XOR operation of two binary numbers because $g_{1}(1,1)=g_{1}(0,0)=0$ and $g_{1}(0,1)=1$. For more than 2 binary parameters, this function still performs in the same way as the XOR operation. For example, $g_{1}(0,1,0)=g_{1}\left(g_{1}(0,1), 0\right)=1$ and $g_{1}(0,1,0,1)=g_{1}\left(g_{1}\left(g_{1}(0,1), 0\right), 1\right)=0$. Therefore, the XOR result of the neighboring variable node values of check node $j$ can be formulated as $g_{1}\left(\mathbf{P}_{j} \mathbf{x}\right)$. For any binary vector with even weight as input, the function value is 0 , and for any binary vector with odd weight as input, the function value is 1 . It can be verified that the function value is larger than 0 and less than 1 for any other points in the range $[0,1]^{2}$. As a result, the value of $g_{1}\left(\mathbf{P}_{j} \mathbf{x}\right)$ is zero for a satisfied check node and positive for an unsatisfied check node. So the penalty coefficient should be positive to penalized the non-codeword. 
In order to derive the updating rules of $x_{i}$ in (5.4) with CN-penalty function $g_{1}(\mathbf{x})$, we need to find the partial derivative of $g_{1}\left(\mathbf{P}_{j} \mathbf{x}\right)$ with respect to $x_{i}$. If $x_{i} \in N_{j}$, based on Property 2, we know $g_{1}\left(\mathbf{P}_{j} \mathbf{x}\right)=g_{1}\left(g_{1}\left(\mathbf{P}_{j} \mathbf{x} \backslash x_{i}\right), x_{i}\right)=g_{1}\left(\mathbf{P}_{j} \mathbf{x} \backslash x_{i}\right)+x_{i}-$ $2 g_{1}\left(\mathbf{P}_{j} \mathbf{x} \backslash x_{i}\right) x_{i}$, where $\mathbf{P}_{j} \mathbf{x} \backslash x_{i}$ indicates all components of $\mathbf{P}_{j} \mathbf{x}$ except $x_{i}$. Then it can be derived $\frac{\partial g_{1}\left(\mathbf{P}_{j} \mathbf{x}\right)}{\partial x_{i}}=1-2 g_{1}\left(\mathbf{P}_{j} \mathbf{x} \backslash x_{i}\right)$. This combined with the equation $g_{1}\left(\mathbf{P}_{j} \mathbf{x} \backslash x_{i}\right)=$ $\frac{g_{1}\left(\mathbf{P}_{j} \mathbf{x}\right)-x_{i}}{1-2 x_{i}}$ gives

$$
\frac{\partial g_{1}\left(\mathbf{P}_{j} \mathbf{x}\right)}{\partial x_{i}}=\frac{2 g_{1}\left(\mathbf{P}_{j} \mathbf{x}\right)-1}{1-2 x_{i}} .
$$

Let $t=2 g_{1}\left(\mathbf{P}_{j} \mathbf{x}^{k}\right)-1$, the updating equation for $E_{j \rightarrow i}^{k+1}$ in Algorithm 4 is

$$
E_{j \rightarrow i}^{k+1}=\left(z_{j(i)}^{k+1}-\lambda_{j(i)}^{k+1}-\frac{\alpha}{\mu} \frac{t}{1-2 x_{i}^{k}}\right),
$$

where $\alpha$ is the penalty coefficient. The value of $t$ can be reused for all variable nodes connected to the same check node.

2)

$$
g_{2}\left(x_{1}, x_{2}, \ldots, x_{n}\right)=\prod_{i}^{n}\left(1-2 x_{i}\right) .
$$

This penalty function also satisfies all the requirements. For binary vectors containing an even number of ones, the function value is 1 . For binary vectors containing an odd number of ones, the function value is -1 . For a vector with components in the range $(0,1)$, its function value is larger than -1 and less than 1 . In order to add the objective function value for solutions that cannot satisfy the parity-checks, the penalty coefficient should be negative.

The updating rule of $x_{i}$ can also be derived based on Property 2. If $x_{i} \in N_{j}$, we have that $g\left(\mathbf{P}_{j} \mathbf{x}\right)=g\left(g\left(\mathbf{P}_{j} \mathbf{x} \backslash x_{i}\right), x_{i}\right)=g\left(\mathbf{P}_{j} \mathbf{x} \backslash x_{i}\right)\left(1-2 x_{i}\right)$. Then

$$
\frac{\partial g\left(\mathbf{P}_{j} \mathbf{x}\right)}{\partial x_{i}}=-2 g\left(\mathbf{P}_{j} \mathbf{x} \backslash x_{i}\right)=-2 \frac{g\left(\mathbf{P}_{j} \mathbf{x}\right)}{1-2 x_{i}} .
$$

Let $t=g\left(\mathbf{P}_{j} \mathbf{x}^{k}\right)$, the updating equation for $E_{j \rightarrow i}^{k+1}$ in Algorithm 4 is

$$
E_{j \rightarrow i}^{k+1}=\left(z_{j(i)}^{k+1}-\lambda_{j(i)}^{k+1}+\frac{\alpha}{\mu} \frac{2 t}{1-2 x_{i}^{k}}\right),
$$

where $\alpha$ is the penalty coefficient.

It can be verified that $g_{2}(\mathbf{x})=1-2 g_{1}(\mathbf{x})$ for vectors of all dimensions. Suppose $\alpha_{1}$ and $\alpha_{2}$ are the penalty coefficients for CN-penalty functions $g_{1}(\mathbf{x})$ and $g_{1}(\mathbf{x})$, 
respectively, we have $\boldsymbol{\gamma}^{T} \mathbf{x}+\alpha_{2} \sum_{j=1}^{m} g_{2}\left(\mathbf{P}_{j} \mathbf{x}\right)=\boldsymbol{\gamma}^{T} \mathbf{x}+\alpha_{2} \sum_{j=1}^{m}\left(1-2 g_{1}\left(\mathbf{P}_{j} \mathbf{x}\right)\right)=$ $\boldsymbol{\gamma}^{T} \mathbf{x}+\left(-2 \alpha_{2}\right) \sum_{j=1}^{m} g_{1}\left(\mathbf{P}_{j} \mathbf{x}\right)+\alpha_{2}$. Since the constant term $\alpha_{2}$ in the objective function does not affect the decoding result, we conclude that the decoding performance of (5.4) with CN-penalty function $g_{1}(\mathbf{x})$ and penalty coefficient $\alpha_{1}=-2 \alpha_{2}$ is the same as that with CN-penalty function $g_{2}(\mathbf{x})$ and penalty coefficient $\alpha_{2}$.

3) For binary vectors, $g_{1}(\mathbf{x})$ is equivalent to the XOR operation. Since the binary XOR operation is simple, we force the arguments of $g_{1}(\mathbf{x})$ to be binary by using the hard decision value of $\mathbf{x}$ instead of $\mathbf{x}$. The function $g_{1}(\mathbf{x})$ with binary arguments is indicated by $g_{3}(\mathbf{x})$. Since $g_{3}(\mathbf{x})$ is not differentiable, we cannot derive the update rule directly from it. The same update rule as $g_{1}(\mathbf{x})$ is used, but in a simpler form.

Let $\hat{\mathbf{x}}$ denote the hard-decision value of $\mathbf{x}$. Then $t^{\prime}=g_{1}\left(\mathbf{P}_{j} \hat{\mathbf{x}}\right)$ is the XOR of all elements of $\mathbf{P}_{j} \hat{\mathbf{x}}$. By replacing $t$ in (5.9) with $1-2 t^{\prime}$, we obtain the following updating equation for $E_{j \rightarrow i}$ :

$$
E_{j \rightarrow i}^{k+1}=\left\{\begin{array}{ll}
l l z_{j(i)}^{k+1}-\lambda_{j(i)}^{k+\frac{\alpha}{\mu}}, & \text { if } t^{\prime} \oplus \hat{x}_{i}=0 \\
z_{j(i)}^{k+1}-\lambda_{j(i)}^{k+1}+\frac{\alpha}{\mu}, & \text { if } t^{\prime} \oplus \hat{x}_{i}=1
\end{array},\right.
$$

where $\alpha$ is the penalty coefficient and $\oplus$ is the XOR operation.

Inside the Algorithm 4, the hard-decision values of variables $x_{i}^{k+1}$ are obtained in line 16. If we re-arrange Algorithm 4 and move the calculation of each paritycheck inside the update of each check node before line 6 we can re-use these available calculation results.

\subsection{Computational Complexity Analysis}

The penalty terms introduce additional complexity to ADMM decoding. Here we calculate and compare the number of extra computations introduced by the different penalty functions in one iteration. For simplicity, the ADMM CN-penalized decoder with function $g_{1}(\mathbf{x}), g_{2}(\mathbf{x})$ and $g_{3}(\mathbf{x})$ are denoted by ADMM-CNP-g1 decoder, ADMM-CNP-g2 decoder, and ADMM-CNP-g3 decoder, respectively. ADMM-VNPh1 decoder and ADMM-VNP-h2 decoder indicate the ADMM VN-penalized decoder with function $h_{1}(x)$ and $h_{2}(x)$, respectively. We do not count the multiplication with 2 as a multiplication because it can be implemented by bit shifting.

For the ADMM-CNP-g1 decoder, the calculation of $g_{1}\left(\mathbf{P}_{j} \mathbf{x}\right)$ involves $2\left(d_{j}-1\right)$ 


\begin{tabular}{|c|c|c|c|c|c|}
\hline & ADMM-VNP-h1 & ADMM-VNP-h2 & ADMM-CNP-g1 & ADMM-CNP-g2 & ADMM-CNP-g3 \\
\hline comparison & $n$ & 0 & 0 & 0 & $\sum_{j=1}^{m} d_{j}$ \\
\hline addition & $n$ & $n$ & $\sum_{j=1}^{m}\left(4 d_{j}-1\right)+1$ & $\sum_{j=1}^{m}\left(3 d_{j}\right)$ & $\sum_{j=1}^{m} d_{j}$ \\
\hline multiplication & 0 & 0 & $\sum_{j=1}^{m}\left(3 d_{j}-1\right)$ & $\sum_{j=1}^{m}\left(3 d_{j}-1\right)$ & 0 \\
\hline
\end{tabular}

Table 5.1: Additional operation per iteration introduced by different penalty functions.

additions and $d_{j}-1$ multiplications. Then $t=2 g_{1}\left(\mathbf{P}_{j} \mathbf{x}^{k}\right)-1$ involves 1 more addition. The outgoing check node messages $E_{j \rightarrow i}^{k+1}$ introduce $2 d_{j}$ extra additions and $2 d_{j}$ extra multiplications. Totally, there will be $\sum_{j=1}^{m}\left(4 d_{j}-1\right)+1$ extra additions and $\sum_{j=1}^{m}\left(3 d_{j}-1\right)$ extra multiplications in one iteration of the decoding. For the ADMM-CNP-g2 decoder, the calculation of $t=g_{2}\left(\mathbf{P}_{j} \mathbf{x}\right)$ involves $d_{j}$ additions and $d_{j}-1$ multiplications. The updating of message $E_{j \rightarrow i}^{k+1}$ introduces $2 d_{j}$ extra additions and $2 d_{j}$ extra multiplications. Totally, there will be $\sum_{j=1}^{m}\left(3 d_{j}\right)$ extra additions and $\sum_{j=1}^{m}\left(3 d_{j}-1\right)$ extra multiplications in one iteration of the decoding. For the ADMM-CNP-g3 decoder, the calculation of $t$ is already available and therefore, does not require any more operations. Most of the extra addition and multiplication operations are replaced by binary XOR operations which can be implemented efficiently. As a result, there is only $\sum_{j=1}^{m} d_{j}$ additional addition operations and $\sum_{j=1}^{m} d_{j}$ comparison operations remaining in one iteration of the decoding. For the ADMM VN-penalized decoding in [66], the $h_{1}$ penalty terms introduce $n$ addition operations and $n$ comparison operations in one iteration. The $h_{2}$ penalty terms introduce $n$ addition operations and 0 multiplication operations in one iteration.

All the information is summarized in Table 5.1. It shows that the ADMM-CNP-g2 decoder is simpler than the ADMM-CNP-g1 decoder while maintaining the same error correction performance. The ADMM-CNP-g3 decoder does not require multiplication and is much simpler than the ADMM-CNP-g1 decoder. It also shows that ADMM CN-penalty functions generally are more complex than the ADMM VN-penalty functions. The high complexity comes from the fact that a single variable node will be involved in all penalty terms corresponding to its neighboring check nodes. In the simulation results section, it will be shown the higher complex brings better error correction performance. 


\subsection{Instanton Analysis}

The error correction performance of LDPC codes at high SNR values is difficult to be obtained by Monte Carlo simulations because of the massive amount of calculations and limited computational resources. Different methods have been proposed to estimate the FER performance at high SNR values, such as importance sampling [101,102], and the linear state-space model [103]. They are all based on the knowledge of trapping sets which are small structures in the Tanner graph of the LDPC code that lead the decoder to be trapped and fail to decode. Contrarily, another technique, called instanton analysis, was introduced in [91]. The authors borrowed the idea from physics and defined instantons of error correction codes as the set of most probable noise configurations which lead to decoding failures. Instantons contribute the most decoding errors at high SNR values. Therefore, the minimum norm of the instantons can be used to predict the slope of the FER curve at high SNR values $[104,105]$. More specifically, the FER curve can be approximated by

$$
\exp \left(-\|\mathbf{i n s}\|_{2}^{2} s^{2} / 2\right),
$$

where ins is the instanton with the minimum norm for a given decoder and $s^{2}$ is the SNR value on a linear scale. This approximation can be more accurate if the multiplicity and the curvature factor of the instantons are counted. However, the study of the curvature factor is not conducted in the literature. So it would not be considered here. As pointed in [105], one may assume that the multiplicity is equal to 1 . Therefore, the slope of (5.14) gives a reasonable estimation of the slope of the FER curve.

Several algorithms have been proposed to find the instantons. For example, the general amoeba algorithm was used in [91]. It can be generalized to different channels and decoders. An efficient pseudo-codeword and instanton search algorithm for LP decoding in the AWGN channel was proposed in [106] based on the theoretical relation between the instanton and the decoder output. The instanton search algorithm for LP decoding in the binary symmetric channel was proposed in [107]. Two instanton search algorithms for the ADMM VN-penalized decoder was presented in [66].

Here we briefly introduce the instanton search algorithm ISA-PD in [66] which tries to solve an optimization problem. Assuming that the all-zero codeword is transmitted, the channel noise is $\mathbf{n}$, and the output of the ADMM VN-penalized decoder 
is $\mathbf{w}$, we conclude that $\mathbf{w}$ must have the less objective value than all the other points in the feasible region of the ADMM VN-penalized decoding model, including the transmitted all-zero codeword. This implies the inequality $\gamma(\mathbf{n})^{T} \mathbf{w}+\sum_{i} h\left(w_{i}\right) \leq$ $\gamma(\mathbf{n})^{T} \mathbf{0}+n h(0)$, where $\boldsymbol{\gamma}(\mathbf{n})$ is the LLR vector with respect to the channel noise $\mathbf{n}$. The term $\gamma(\mathbf{n})^{T} \mathbf{0}$ in the right-hand side can be dropped since its value is always 0 . Searching for an instanton is to find the noise configuration leading to a decoding error with the minimum norm. Therefore, the optimization model is

$$
\begin{aligned}
\operatorname{minimize} & \|\mathbf{n}\|_{2}^{2} \\
\text { subject to } & \gamma(\mathbf{n})^{T} \mathbf{w}+\sum_{i=1}^{n} h\left(w_{i}\right) \leq n g(0) .
\end{aligned}
$$

For a non-zero output $\mathbf{w}$ of the ADMM VN-penalized decoder, this model gives the minimum noise configuration $\mathbf{n}$ which can lead the decoder to conclude $\mathbf{w}$ has a less or equal objective value than the all-zero codeword. Therefore, vector $\mathbf{n}$ can result in a decoding error and is the candidate of the instanton. Then this vector $\mathbf{n}$ will be used as the channel noise and applied to the transmitted all-zero code to generate a new channel output. The ADMM VN-penalized decoder will decode this channel output. This usually results in a non-zero result closer to the all-zero codeword than w. The new decoding result can be used to find the noise configuration leading to this decoding result based on the same model. These steps are conducted iteratively, and the noise configuration searched in each iteration will be recorded. In the end, the noise configuration with the minimum norm will be output as the instanton. The result of this iterative searching procedure is related to the first non-zero output of the decoder. Therefore, this procedure should be repeated multiple times with different initialization.

Based on the similar ISA-PD instanton searching framework, we derive an instanton search algorithm for the ADMM CN-penalized decoder. We solve the following optimization problem:

$$
\begin{aligned}
\operatorname{minimize} & \|\mathbf{n}\|_{2}^{2} \\
\text { subject to } & \boldsymbol{\gamma}(\mathbf{n})^{T} \mathbf{w}+\sum_{j=1}^{m} \alpha g\left(\mathbf{P}_{j} \mathbf{w}\right) \leq \alpha m g(\mathbf{0})
\end{aligned}
$$

Similarly, we assume that the all-zero codeword is transmitted, the channel noise is $\mathbf{n}$ and the output of the ADMM CN-penalized decoder is $\mathbf{w}$. The constraint of the model is derived from the fact that the objective value of the ADMM CN-penalized 
decoding model for a decoding output $\mathbf{w}$ is less than or equal to that for the all-zero codeword. For the AWGN channel with BPSK modulation, the received symbol is $y_{i}=1+n_{i}$ because symbol 0 is transmitted. The noise component with a positive value increases the reliability of transmitting a 0 and would not contribute to the decoding error. Therefore, we constrain the noise to be negative. Equivalently, to be consistent with [66], we use $n_{i} \geq 0$ and $y_{i}=1-n_{i}$. Then for the AWGN channel with noise covariance $\sigma^{2}$, the LLR vector is $\gamma(\mathbf{n})=\frac{2}{\sigma^{2}}(\mathbf{1}-\mathbf{n})$ when all-zero codeword is transmitted.

The Lagrangian for this model is

$$
L=\|\mathbf{n}\|_{2}^{2}+\lambda\left(\gamma(\mathbf{n})^{T} \mathbf{w}+\alpha \sum_{j=1}^{m} g\left(\mathbf{P}_{j} \mathbf{w}\right)-\alpha m g(\mathbf{0})\right) .
$$

By making the partial derivatives of $L$ with respect to $\mathbf{n}$ and $\lambda$ equal to 0 , we obtain the equations

$$
\left\{\begin{array}{l}
2 \mathbf{n}-\frac{2 \lambda}{\sigma^{2}} \mathbf{w}=0 \\
\frac{2}{\sigma^{2}}(\mathbf{1}-\mathbf{n})^{T} \mathbf{w}+\alpha \sum_{j=1}^{m} g\left(\mathbf{P}_{j} \mathbf{w}\right)-\alpha m g(\mathbf{0})=0 .
\end{array}\right.
$$

By solving these equations, the solution to (5.16) is

$$
\mathbf{n}^{*}=\mathbf{w}\left(\frac{1}{\|\mathbf{w}\|_{2}^{2}}\left[\|\mathbf{w}\|_{1}-\frac{\alpha \sigma^{2}}{2}\left(m g(\mathbf{0})-\sum_{j=1}^{m} g\left(\mathbf{P}_{j} \mathbf{w}\right)\right)\right]\right) .
$$

The whole instanton search algorithm for the ADMM CN-penalized decoder is summarized in Algorithm 5. Three points should be emphasized, like in [66]. Firstly, after obtaining $\mathbf{n}^{*}$, we have to find the smallest scaling factor $\alpha$ such that the decoder fails with noise $\alpha \mathbf{n}^{*}$ because $\mathbf{n}^{*}$ is an approximation of the noise configuration. The decoder can still fail because of a noise vector with a smaller norm. Secondly, the instanton search algorithm for the ADMM CN-penalized decoder may not converge. The norm of the instanton is not necessarily decreased with the iterations. So we only use the maximum number of iterations as the termination condition. Lastly, the algorithm should be run enough times with different initialization to obtain statistics on instantons for the ADMM CN-penalized decoders. 


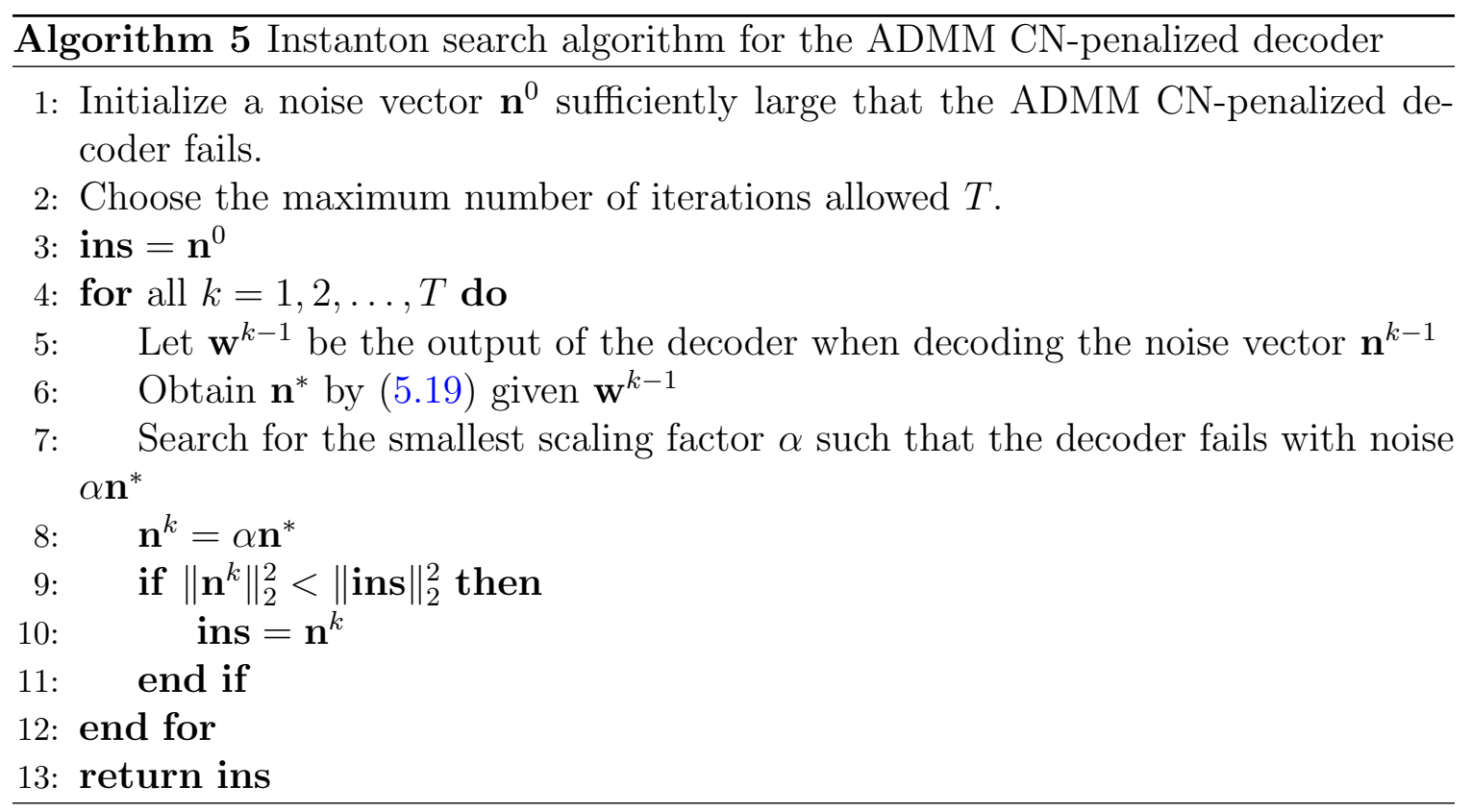

\subsection{Simulation Results}

In this section, we first show the effects of the different parameters in ADMM CNpenalized decoding. Then we present the FER performance for our proposed ADMM CN-penalized decoders and compare them with other decoders. Last, we conduct experiments on instanton and give the instanton analysis on the FER performance for the proposed decoder at high SNR values. All simulations are performed over the AWGN channel with BPSK modulation. All points of the FER curves are obtained by collecting 100 errors. As we conclude before, the decoding performance of the ADMM-CNP-g1 decoder with penalty coefficient $\alpha_{1}$ is the same as the ADMM-CNPg2 decoder with a penalty coefficient $\alpha_{2}$ if $\alpha_{1}=-2 \alpha_{2}$. Since the penalty coefficient of $g_{1}(\mathbf{x})$ is positive, which is better for the comparison with the ADMM VN-penalized decoder, the ADMM-CNP-g2 decoder will not be simulated here although it is simpler than the ADMM-CNP-g1 decoder.

\subsubsection{Selection of Parameters}

Several parameters are involved in the ADMM CN-penalized decoder. They include the Lagrangian coefficient $\mu$, the penalty coefficient $\alpha$, and the maximum number of iterations $I_{\text {max }}$. The code we used here is the $(576,288)$ code $\mathcal{C}_{1}$ from the IEEE 802.16e standard [4]. 


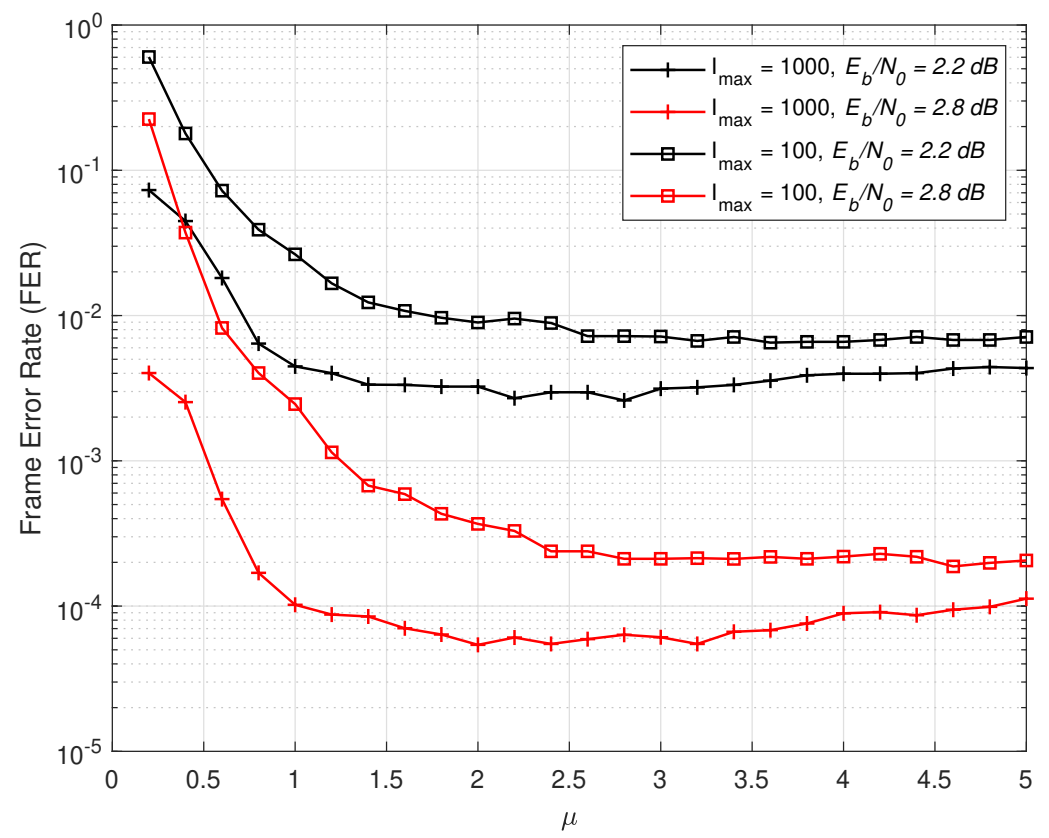

Figure 5.1: FER performance of $\mathcal{C}_{1}$ under the ADMM-CNP-g1 decoder plotted as a function of parameter $\mu$, the Lagrangian parameter.

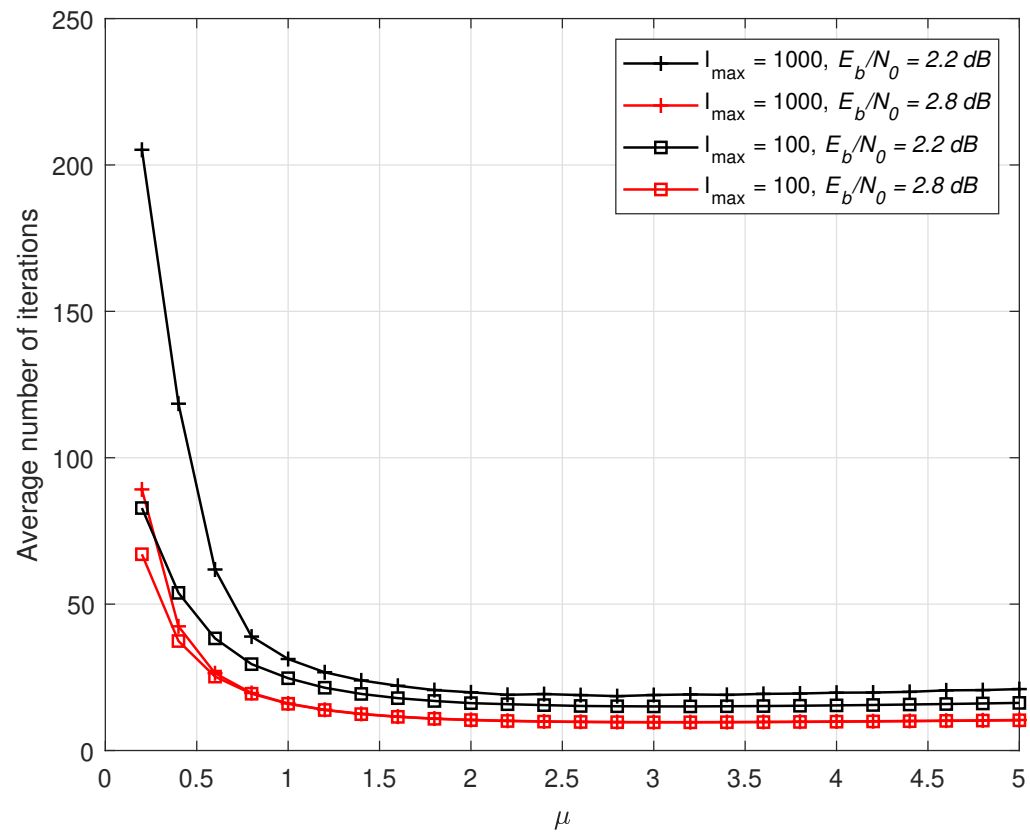

Figure 5.2: Average number of iterations of $\mathcal{C}_{1}$ under the ADMM-CNP-g1 decoder plotted as a function of parameter $\mu$, the Lagrangian parameter. 


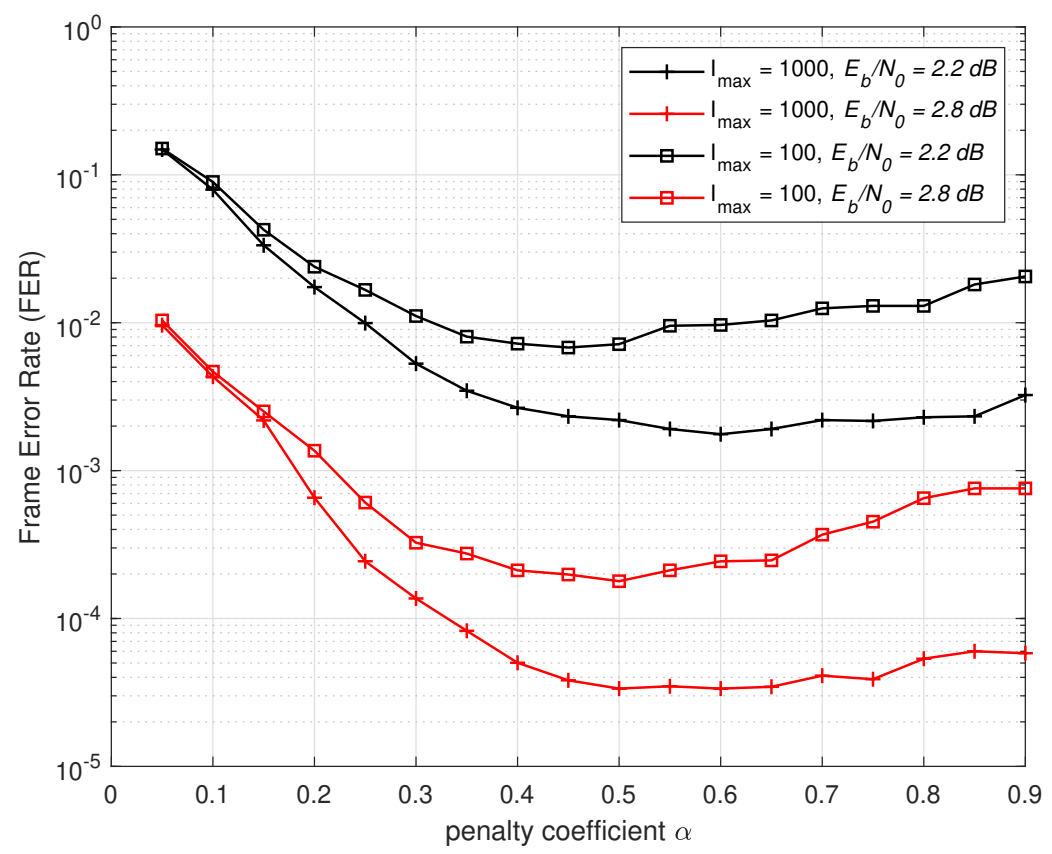

Figure 5.3: FER performance of $\mathcal{C}_{1}$ under the ADMM-CNP-g1 decoder plotted as a function of $\alpha$, the penalty parameter.

In Fig. 5.1, we plot the FER performance of $\mathcal{C}_{1}$ as a function of the Lagrangian coefficient $\mu$ for the ADMM-CNP-g1 decoder. The penalty coefficient $\alpha$ is 0.4 . For $I_{\max }=100$, the two curves show that the performance of the decoder is stable for $\mu \geq 2.5$. For $I_{\max }=1000$, the FER of the decoder is decreased sharply before $\mu=1.5$ and starts to increase for $\mu \geq 3.8$. We also plot the average number of iterations as a function of $\mu$ for the ADMM-CNP-g1 decoder in Fig. 5.2. It shows that for different $I_{\max }$ and $E_{b} / N_{0}$ values, the average decoding time should be almost the same if $\mu \geq 1.5$. Since $g_{3}(\mathbf{x})$ is actually a special case of $g_{1}(\mathbf{x})$ with binary arguments, their sensitivity to different parameters are similar. So they should share the same penalty coefficient. The same experiments on the ADMM-CNP-g3 decoder verify this. We will not show the simulation curves and emphasis this conclusion again. In the end, we choose 2.8 as the optimal value of $\mu$ for $\mathcal{C}_{1}$.

In Fig. 5.3, we plot the FER performance of $\mathcal{C}_{1}$ as a function of the penalty coefficient $\alpha$ for the ADMM-CNP-g1 decoder. For different $I_{\max }$ and $E_{b} / N_{0}$ values, all curves show that the FER of the decoder decreases first and then start to increase. Two points should be noted. Firstly, for the smaller $I_{\max }$, the performance of the decoder is more sensitive to the penalty coefficient, and the optimal range is usually 


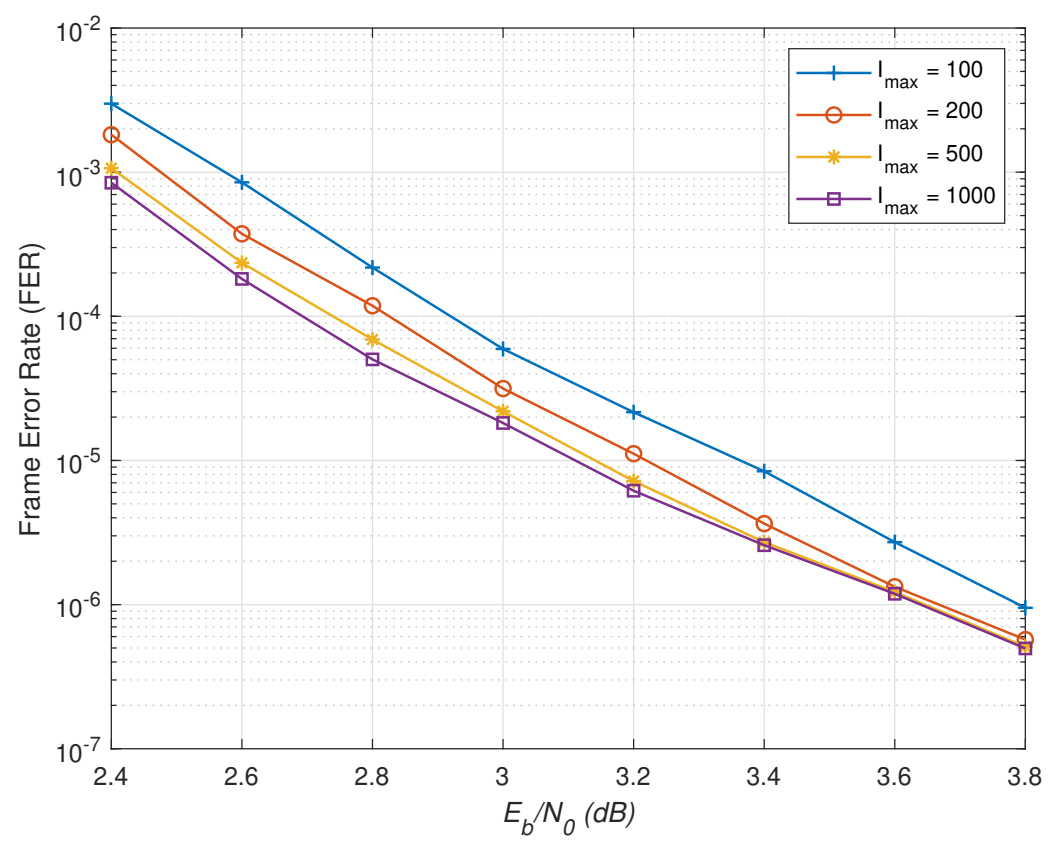

Figure 5.4: FER performance of $\mathcal{C}_{1}$ under the ADMM-CNP-g1 decoder plotted as a function of $I_{\max }$, the maximum number of iterations.

smaller. Secondly, for the smaller $I_{\max }$, the optimal value of the penalty coefficient $\alpha$ is smaller. In sum, the optimal value of $\alpha$ should be around $[0.4,0.45]$ for $\mathcal{C}_{1}$ and we choose 0.4 . For $\mathcal{C}_{1}$, there are only two different check node degree 6 and 7 . This limits the performance gain if we assign different penalty coefficients for check nodes with different degrees.

In Figs. 5.1 and 5.3, the effect of the number of maximum iterations $I_{\max }$ has already been shown for low $E_{b} / N_{0}$ values. Obviously, the larger $I_{\text {max }}$ improves the FER performance. In Fig. 5.4, we give the FER performance of $\mathcal{C}_{1}$ for the ADMMCNP-g1 decoder with different $I_{\text {max }}$ at higher $E_{b} / N_{0}$ values. It shows the performance gap decreases with the increasing $E_{b} / N_{0}$. Only around half of the errors can be further corrected if $I_{\max }$ is increased from 100 to 1000 at $3.8 \mathrm{~dB}$. We can conclude that the maximum number of iterations $I_{\max }$ has a larger effect on the FER performance when the $E_{b} / N_{0}$ is small. 


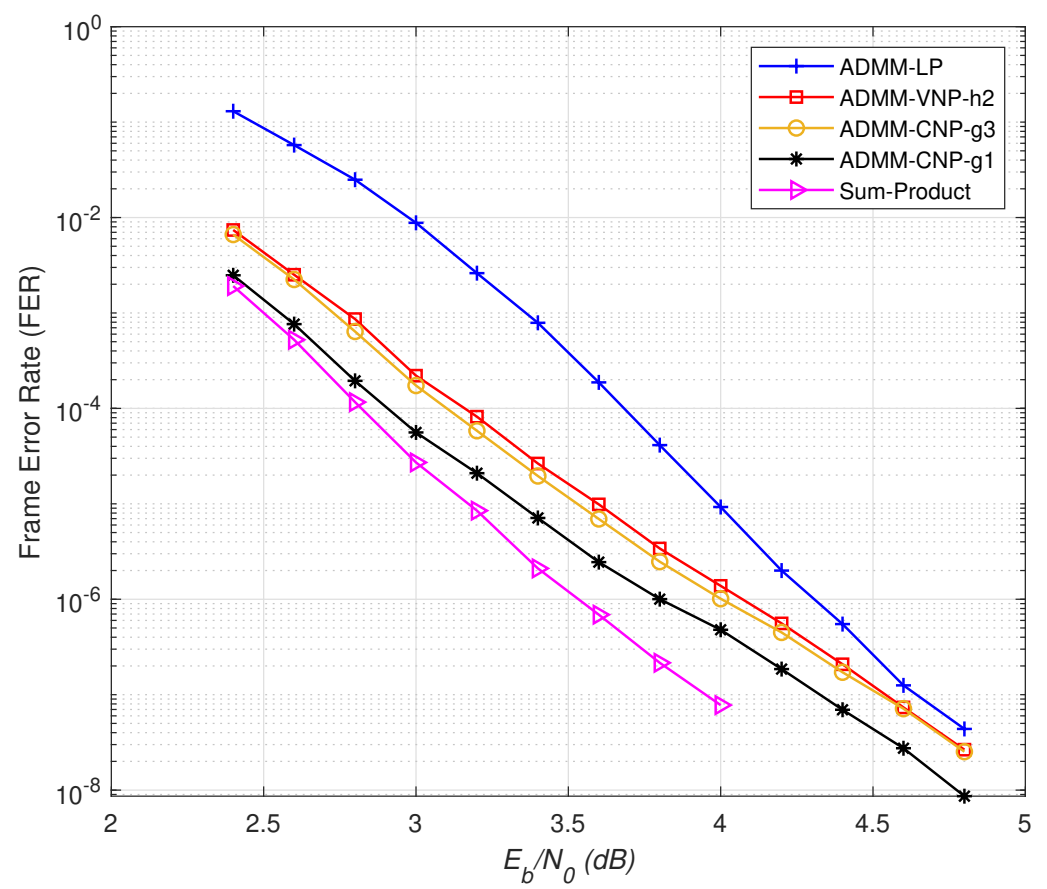

Figure 5.5: FER performance of $\mathcal{C}_{1}$ under the proposed ADMM CN-penalized decoders and other decoders.

\subsubsection{FER Performance}

In this part, we present the simulation result of the proposed ADMM CN-penalized decoders with optimized parameters. The ADMM-LP decoding in [33], the ADMM VN-penalized decoding with $h_{2}(x)$ penalty terms in [66] and the Sum-Product decoding are used here to make a comparison. For a fair comparison, the maximum number of iterations is selected to be 100 for all algorithms. The over-relaxation coefficient is set to 1.9 for ADMM (penalized) decoding algorithms, and the Lagrangian parameter $\mu$ is set to the same number for all three ADMM (penalized) decoding algorithms.

Fig. 5.5 shows the FER performance of $\mathcal{C}_{1}$ for the different decoders. The Lagrangian parameter $\mu$ is set to 2.8. The penalty coefficient for the ADMM-VNP-h2 decoder is $\alpha=1.9$. The penalty coefficient for the two proposed ADMM-CNP decoders is $\alpha=0.4$. It can be seen that the ADMM-CNP-g1 decoder improves the performance of the ADMM-LP decoder by more than two orders of magnitude when $E_{b} / N_{0}$ is larger than $2.6 \mathrm{~dB}$ and less than $3.6 \mathrm{~dB}$. The improvement is still more than one order of magnitude for $E_{b} / N_{0}$ as large as $4.2 \mathrm{~dB}$. Compared with the ADMM-VNP-h2 decoder, our proposed ADMM-CN-penalized decoder has a better performance at all 


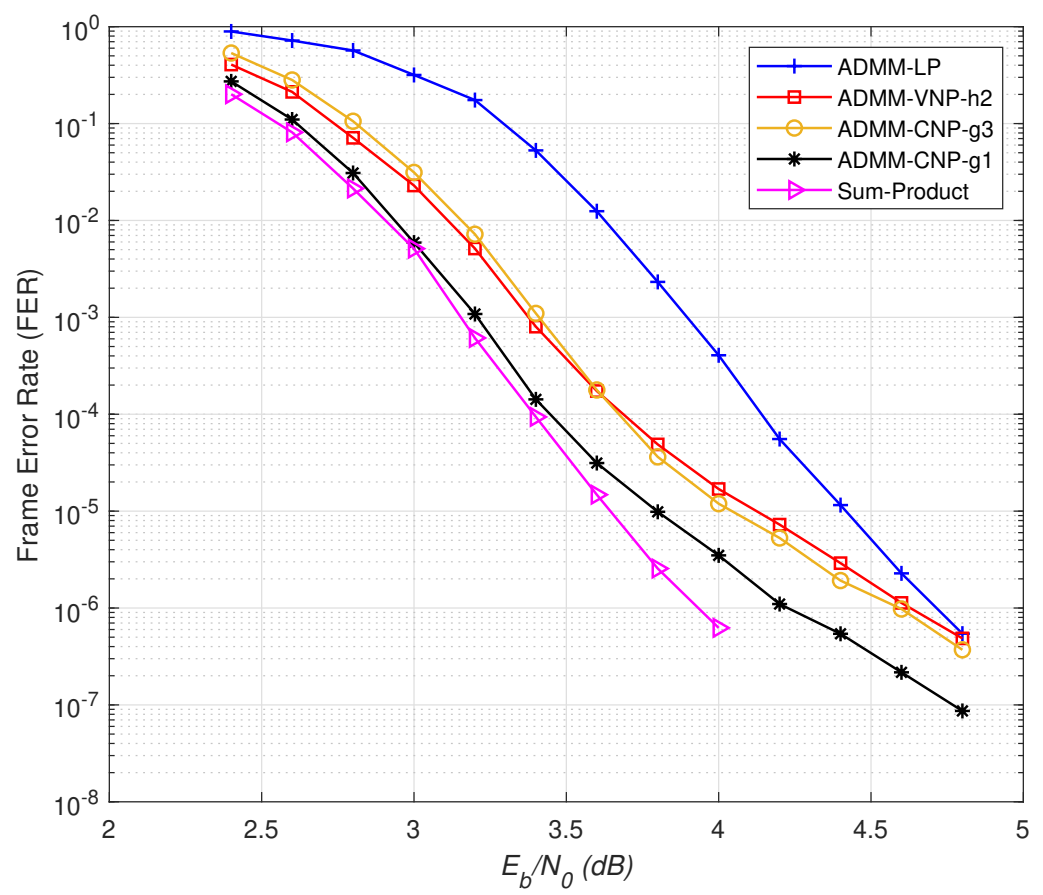

Figure 5.6: FER performance of $\mathcal{C}_{7}$ code under the proposed ADMM CN-penalized decoders and other decoders.

$E_{b} / N_{0}$ values. The simplified ADMM-CNP-g3 decoder has inferior performance, but still outperforms the ADMM-VNP-h2 decoder. Despite its poor performance at low $E_{b} / N_{0}$, ADMM-LP decoder has a sharper slope at high $E_{b} / N_{0}$ than all three ADMM penalized decoders, which indicates it may have better performance at high $E_{b} / N_{0}$ values.

Fig. 5.6 shows the FER performance of the $(1152,864)$ code $\mathcal{C}_{7}$ from the IEEE 802.16e standard [4] under the different decoders. The Lagrangian parameter $\mu$ is set to 2.8. The penalty coefficient for the ADMM-VNP-h2 decoder is $\alpha=2.3$. The penalty coefficient for the two proposed decoders is $\alpha=0.5$. It can be seen that the ADMM-CNP-g1 decoder narrows the performance gap between the Sum-Product decoder and ADMM-LP decoder significantly for $E_{b} / N_{0}$ values less than $3.6 \mathrm{~dB}$. For larger $E_{b} / N_{0}$ values, the ADMM-CNP-g1 decoder suffers from an error floor, but its performance improvement compared with the ADMM-LP decoder is still more than one order of magnitude for $E_{b} / N_{0}$ as large as $4.2 \mathrm{~dB}$. Compared with the ADMMVNP-h2 decoder, our proposed ADMM-CNP-g1 decoder has about $0.2 \mathrm{~dB}$ gain for $E_{b} / N_{0}$ larger than $3.2 \mathrm{~dB}$, while the ADMM-CNP-g3 decoder has similar performance. 


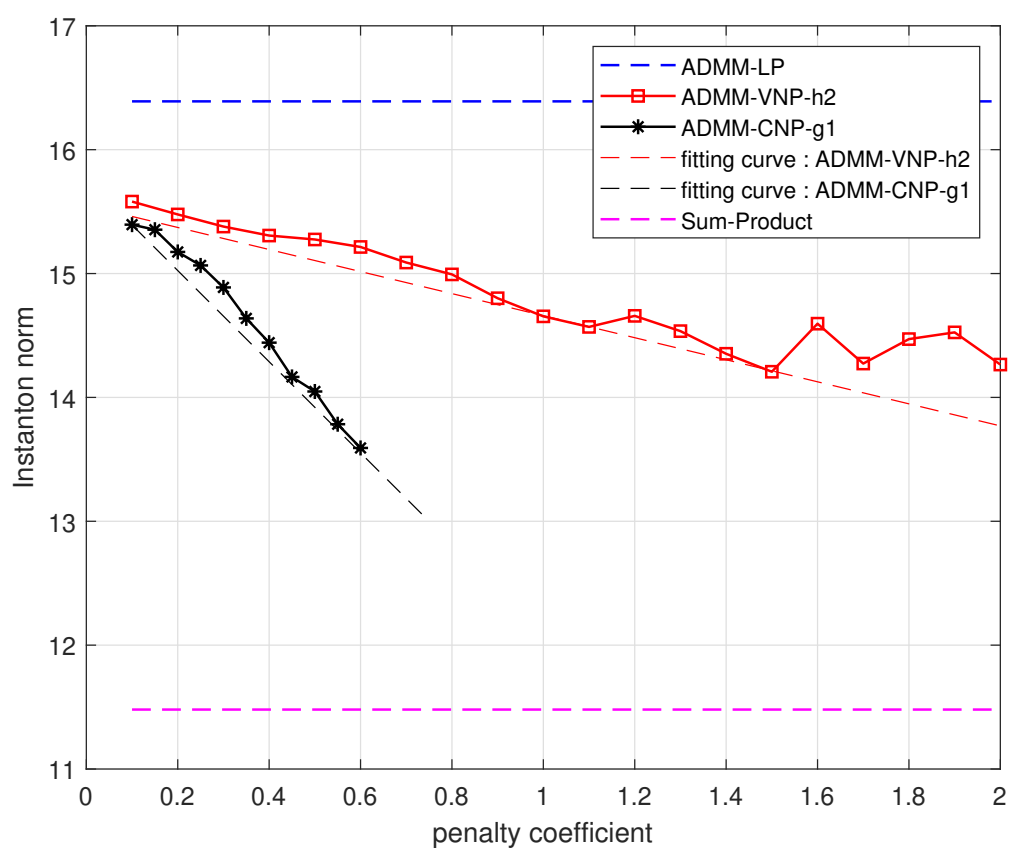

Figure 5.7: The minimum instanton norm of $\mathcal{C}_{8}$ under the ADMM-CNP-g1 decoder as a function of penalty coefficient.

Also, the ADMM-LP decoder has a sharper slope at high $E_{b} / N_{0}$ than all three ADMM penalized decoders, which indicates it may have better performance at high $E_{b} / N_{0}$ values.

\subsubsection{Instanton Estimation}

Our proposed ADMM-CNP-g1 decoder has been shown to perform better than the ADMM-VNP-h2 decoder at all simulated points. However, it is difficult to verify this conclusion by Monte Carlo simulation at higher SNR values. Instead, we use the instanton analysis to estimate and compare the FER performance of the decoders. We first use the instanton search algorithm described in Section 5.4 to find the minimum norm of different decoders' instantons. Then we predict the slop of the decoder in the high SNR region by (5.14). Algorithm 5 is executed 3000 times with different initializations, and the maximum number of iterations we used inside the instanton search algorithm is $T=100$. The maximum number of iterations for three ADMM (penalized) decoding algorithms is also $I_{\max }=100$.

The FER performance of the ADMM-CNP-g1 decoder is affected by the penalty 
coefficient. So the instantons are also related to the penalty coefficient. We searched for the instantons of the ADMM-CNP-g1 decoder with different penalty coefficients. Then we plot the minimum instanton norm as a function of $\alpha$. As suggested in [66], we use the worst-case instanton norms' fitting-curves to obtain a more accurate result.

The first code we used here is the $(155,64)$ Tanner code $\mathcal{C}_{8}$. Fig. 5.7 shows the minimum instanton norm of $\mathcal{C}_{8}$ under the ADMM-CNP-g1 decoder as a function of penalty coefficient $\alpha$. The LP instantons are obtained by the method proposed in [106], and the data of BP decoding is obtained directly from [66]. The Lagrangian coefficient is 3.0. It can be seen that the minimum instanton norms of both the ADMM-CNP-g1 decoder and the ADMM-VNP-h2 decoder decrease with the increase of the penalty coefficients. This implies that a larger penalty coefficient will result in a worse error correction performance in high SNR values. Therefore, the optimal penalty coefficient should be selected as small as possible. We can also find that the slopes of the two fitting curves are different. The decreasing speed of the curve for the ADMM-CNP-g1 decoder is larger than that of the ADMM-VNP-h2 decoder, which implies that the optimal penalty coefficient for the ADMM CN-penalized decoder is smaller. Based on in-advance simulations, the optimal penalty coefficient of the ADMM-CNP-g1 decoder for $\mathcal{C}_{8}$ is 0.4 , and the optimal penalty coefficient of the ADMM-VNP-h2 decoder for $\mathcal{C}_{8}$ is 2.0. This agrees with our conjecture. From Fig. 5.7, we can see that the minimum instanton norm of the ADMM-CNP-g1 decoder at $\alpha=0.4$ is around 14.33 and the minimum instanton norm of ADMM-VNP-h2 at $\alpha=2.0$ is around 13.80 . The minimum norms imply that the ADMM-CNP-g1 decoder will have a sharper FER curve than the ADMM-VNP-h2 decoder at high SNR values.

In Fig. 5.8, we plot the FER asymptotics predicted by the minimum norm instanton as well as Monte Carlo simulation results of $\mathcal{C}_{8}$ for different decoders. The SNR used here is defined as $2 R \frac{E_{b}}{N_{0}}$ on a linear scale, where $R$ is the code rate. The asymptotic curves are calculated by (5.14) with different norms. In the high SNR region, we can see that the FER curves of each decoder are parallel with their asymptotic curves. This shows the effectiveness of the instanton analysis method. The FER performance of the proposed ADMM-CNP-g1 decoder is almost the same as that of the ADMM-VNP-h2 decoder in the low SNR region and starts to show a better slope for SNR larger than 2.75. The FER performance of the ADMM-LP decoder is the worst among all decoders in the low SNR region. It exceeds that of the ADMM-LP, the 


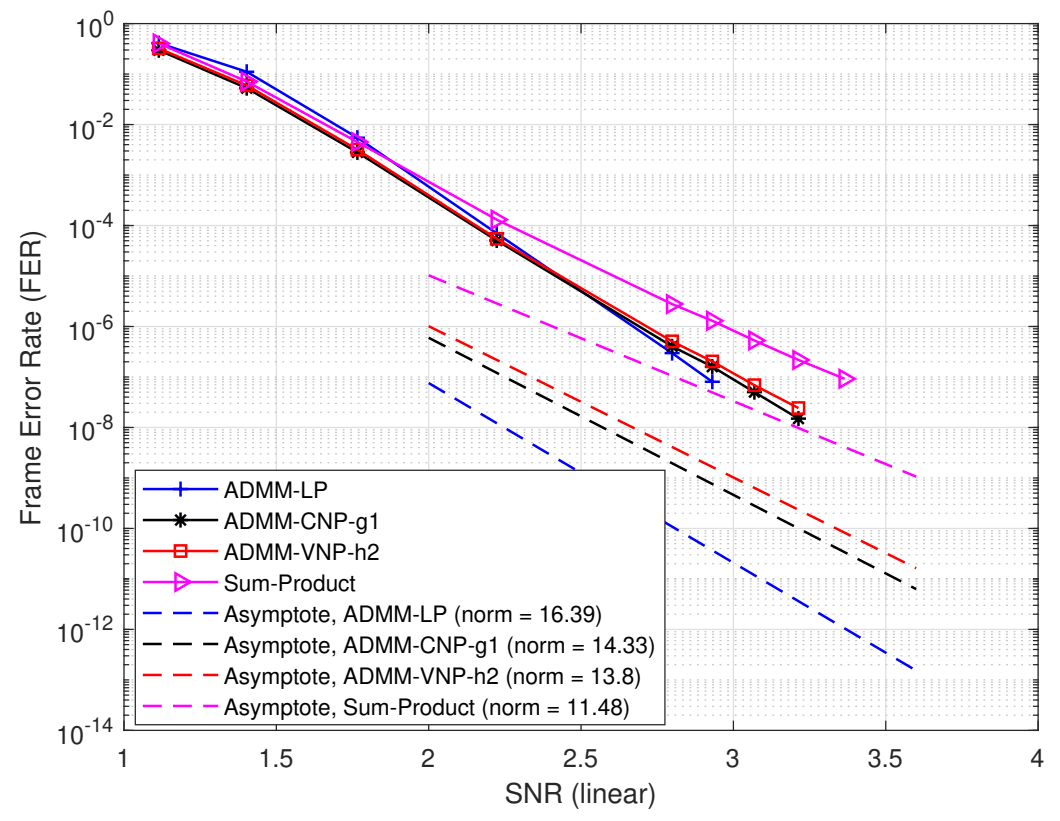

Figure 5.8: FER performance estimations of $\mathcal{C}_{8}$ under the proposed ADMM-CNP-g1 decoder and other decoders. The SNR value used here is on a linear scale.

ADMM-VNP-h2 decoder, and the ADMM-CNP-g1 decoder with the increase of SNR. These facts verify the conclusion that the ADMM-CNP-g1 decoder will have a better slope than the ADMM-VNP-h2 decoder at high SNR values, and the ADMM-LP has the steepest slope at high SNR values. If more information on the multiplicity and the curvature factor of the instantons is known, the asymptote curves can be lifted, resulting in a tighter lower bound. Here due to the missing knowledge, only the slope of the FER curve can be predicted.

The second code we used here is $\mathcal{C}_{7}$. Fig. 5.9 shows the minimum instanton norm of $\mathcal{C}_{7}$ under the ADMM-CNP-g1 decoder as a function of penalty coefficient $\alpha$. The parameter settings are the same as Section 5.5.2. It can be seen that noticeable fluctuations appear in the curves of instanton norms of both ADMM-CNP-g1 decoder and ADMM-VNP-h2 decoder due to limited simulations. However, the overall trend of the two curves and their fitting curves is decreasing with the increase of the penalty coefficients. Also, the decreasing speed of the curve for the ADMM-CNP-g1 decoder is larger than that of the ADMM-VNP-h2 decoder. These observations are the same as for $\mathcal{C}_{8}$, and similar conclusions can be reached. For example, the optimal penalty coefficient of the ADMM-CNP-g1 decoder is 0.5 which is smaller than that of the 


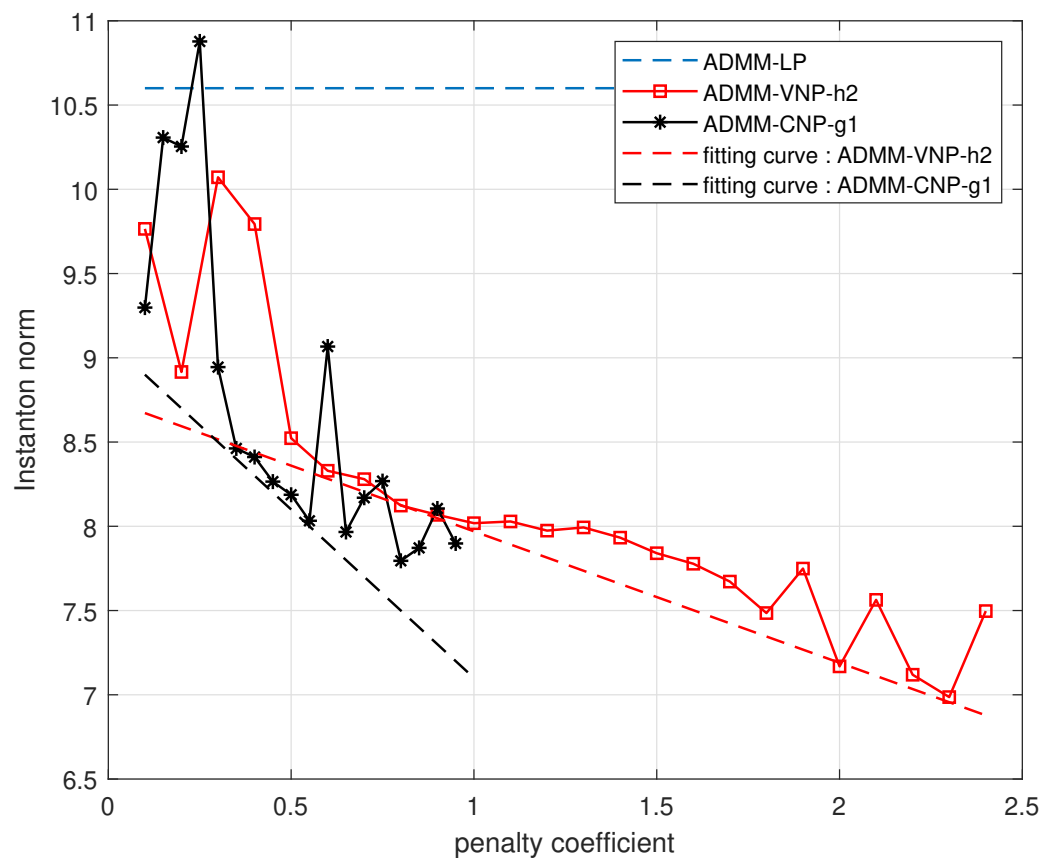

Figure 5.9: The minimum instanton norm of $\mathcal{C}_{7}$ under the ADMM-CNP-g1 decoder as a function of penalty coefficient $\alpha$.

ADMM-VNP-h2 decoder, 2.3. From Fig. 5.7, we can see that the minimum instanton norm of the ADMM-CNP-g1 decoder at $\alpha=0.5$ is around 8.1 and the minimum instanton norm of the ADMM-VNP-h2 decoder at $\alpha=2.3$ is around 7.0. The minimum norms imply the ADMM-CNP-g1 decoder will have a better slope than the ADMM-VNP-h2 decoder.

In Fig. 5.10, we plot the FER asymptotics predicted by the minimum norm instanton as well as Monte Carlo simulation results of different decoders. In the high SNR region, we can see that the FER curves of each decoder are parallel with their asymptotic curves. The slopes of the three curves are different at high SNR values. The ADMM-LP has the steepest slope at high SNR values which means its performance will surpass the other two eventually. Actually, it can be seen that its performance will be better than that of the ADMM-VNP-h2 decoder for SNR larger than 4.5. The slope of the ADMM-CNP-g1 decoder is a little steeper than that of the ADMM-VNP-h2 decoder. Since its current performance is better than the ADMM-VNP-h2 decoder, we can conclude that its performance will be better than the ADMM-VNP-h2 decoder for all SNR values. 


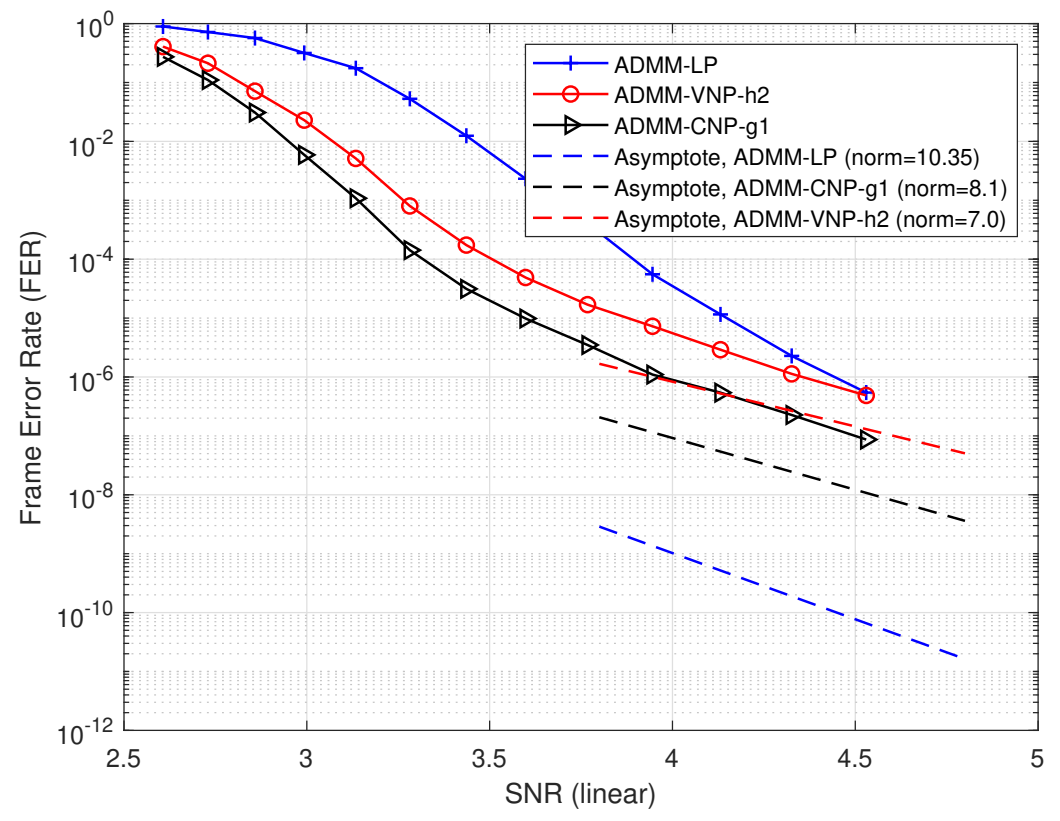

Figure 5.10: FER performance estimations of $\mathcal{C}_{7}$ under the proposed ADMM-CNPg1 decoder and other decoders. The SNR value used here is on a linear scale.

\subsection{Conclusion}

In this chapter, we propose an ADMM CN-penalized decoding algorithm by introducing check-node based penalty terms to the objective of the LP model. These penalty terms penalize the unsatisfied check nodes and improve the FER performance of the original ADMM decoding at low SNR values significantly. The properties of the CNpenalty function are investigated, and several functions that satisfy these properties are proposed. A message-passing style algorithm is given to solve this model, and its convergence properties are studied. Moreover, it is proved that the error correction performance of the decoding algorithm is independent of the transmitted codeword. The FER performance is simulated in the low SNR region and analyzed by instanton in the high SNR region. Simulations show that the ADMM CN-penalized decoding improves the performance of ADMM decoding at low SNR values significantly, and it has better or comparable decoding performance than the ADMM VN-penalized decoding. 


\section{Chapter 6}

\section{Low-Complexity Post-Processing Technique to Lower the Error Floor}

The FER of LDPC codes drops sharply as the noise level decreases in the low-tomedium SNR region because more bits are free of errors and can be used to correct other errors. With the SNR value increases in the high SNR region, although fewer variables nodes are in erroneous states, the decoder cannot recover them if they happen to be inside a trapping set structure as explained in Section 2.2. The trapping sets become the main reason for decoding failures in the high SNR region and can be used to explain the error floor. Some works tried to devise better decoding algorithms that can break the trapping sets. Among them, Cavus et al. proposed to store dominant trapping sets in a look-up table and reverse the erroneous decoding result if one matched syndrome pattern is found in the look-up table [82].

In this chapter, we first review the look-up table based syndrome decoder of [82] in Section 6.1. Then we propose a low-complexity post-processing technique motivated by [82] to lower the error floor of LDPC codes in Section 6.2. The low-complexity post-

processing technique includes an enhanced look-up table based syndrome decoder that can deal with the multiple-matching problem, an efficient syndrome pattern matching (SPM) algorithm for general LDPC codes, and a simplified SPM algorithm for QCLDPC codes. Examples are given to illustrate the construction of the look-up table. The complexities of the proposed algorithm are analyzed in Section 6.3. Simulation results are given in Section 6.4. 


\subsection{Look-Up Table Based Syndrome Decoder}

An $(a, b)$ trapping set can lead to a decoding failure of $a$ erroneous bits and $b$ unsatisfied parity-checks. Therefore, it provides the reason and the characteristic of a possible decoding failure. If we can store the dominant trapping sets, we will have information about the dominant failures and therefore, can identify and correct them instantly. The principle behind the look-up table base syndrome decoder in [82] is using the look-up table to determine if a pre-known failure is detected based on the unsatisfied parity-checks. Once the syndrome pattern matches a pre-stored syndrome pattern, the decoder simply flips the bits in the corresponding error pattern. At this point, all the parity-checks will be satisfied, and the decoder converges to a valid codeword.

The look-up table has $N$ rows and two columns. Each row stores one trapping set. For an $(a, b)$ trapping set, the syndrome pattern is stored in the first column of the row, and the error pattern is stored in the second column of the row. We will use list $\mathcal{L}_{1}$ to indicate the first column of the whole table and $\mathcal{L}_{2}$ to indicate the second column of the whole table. Then $\mathcal{L}_{1}$ includes all the syndrome patterns of pre-known failures and $\mathcal{L}_{2}$ includes all the corresponding error patterns.

It should be noted that the number of trapping sets stored in the table is small in [82]. Total 10 trapping sets are stored for a code of length 96, 8 trapping sets are stored for a code of length 204, and 16 trapping sets are stored for a code of length 2000. The size of the look-up table is so small that the syndrome pattern matching operation cost little expense. In addition, it is not reported in [82] that the trapping sets in the table could have the same syndrome pattern. Therefore, they assumed at most one trapping set could be matched after one decoding failure. However, if we try to extend this technique to other codes, we have to deal with the following situations:

1. When the look-up table has more than the order of $10^{5}$ trapping sets, is there any efficient SPM algorithm to locate the matched item?

2. When more than one item matches the target syndrome pattern, which trapping set should we select to flip the result?

3. When a QC-LDPC code is used, if its isomorphism can be exploited to simplify the SPM algorithm? 


\subsection{Low-Complexity Post-Processing Technique}

In this section, we will try to answer the three questions proposed above. An enhanced look-up table based syndrome decoder is proposed, and two efficient SPM algorithms are presented for general LDPC codes and QC-LDPC codes. We also give some guidelines on constructing the look-up tables for general LDPC codes and QC-LDPC codes.

\subsubsection{Enhanced Look-Up Table Based Syndrome Decoder}

Suppose a codeword $\mathbf{x}$ is transmitted over the channel, and a vector $\mathbf{y}$ is received. The first stage decoder takes vector $\mathbf{y}$ or its LLRs as input and begins to decode. Our proposed decoder will start only if the first stage decoder fails to converge to a valid codeword within the maximum number of iterations. The proposed decoder takes two arguments. The first one is the hard-decision output of the first stage decoder at the end of its iterations which is denoted by $\overline{\mathbf{x}}$. The second argument is the initial channel information which is also the input of the first stage decoder. For simplicity, we assume the channel information is the channel output $\mathbf{y}$. It should be noted, our proposed enhanced look-up table decoder can be applied to the BSC and AWGN channel, and used as the second stage decoder of all types of iterative message-passing decoders.

In our proposed algorithm, dominant trapping sets are also stored in a look-up table, and the syndrome pattern of the previous stage decoder is searched in the table. We define the following strategy to deal with the multiple-matching problem. If several (for example $t$ ) syndrome patterns in the look-up table are matched with the target syndrome pattern, the corresponding codeword bits in all these $t$ trapping sets will be flipped respectively. A sequence of revised vectors $\hat{\mathbf{x}}_{1}, \hat{\mathbf{x}}_{2}, \ldots, \hat{\mathbf{x}}_{t}$ can be obtained, and one of them will be selected as the final revision based on some criteria. Here we use the maximum likelihood criterion. The revised vector owning the maximum likelihood with the channel output will be selected. The details of the look-up table based syndrome decoder are as follows.

In Algorithm 6, the syndrome is calculated by multiplying $\overline{\mathbf{x}}$ with the parity-check matrix and the indices of unsatisfied check nodes (syndrome pattern) are stored in

$\mathbf{s}$. If the length of $\mathbf{s}$ is less than $b_{\max }$, the maximum length of syndrome patterns in the look-up table, we append 0s to make $\mathbf{s}$ have length $b_{\max }$. Then $\mathbf{s}$ is searched 


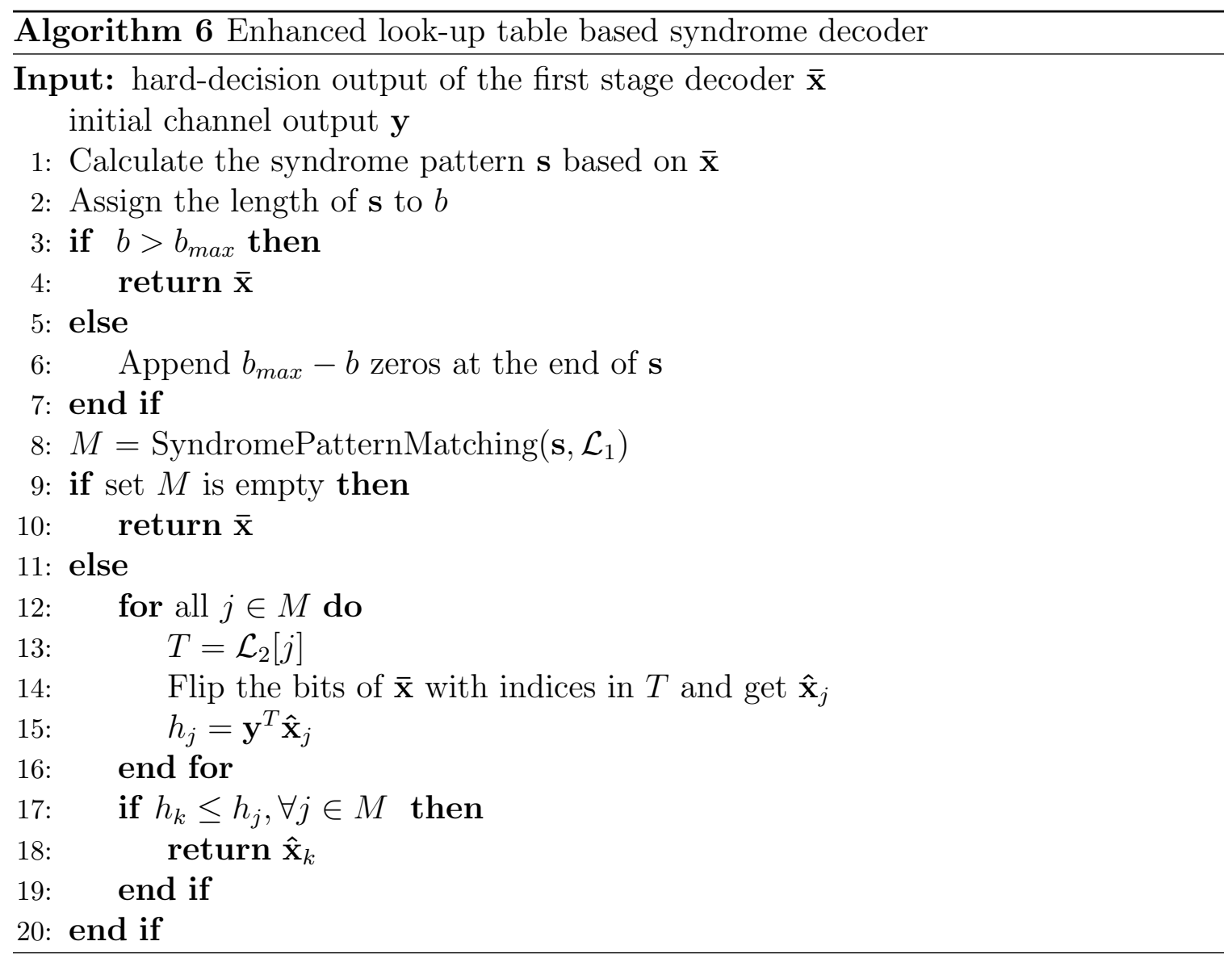


in $\mathcal{L}_{1}$ by an SPM algorithm and the set $M$ stores the indices of all matched items. Based on the indices, their corresponding error pattern information can be found in $\mathcal{L}_{2}$. If $M$ is not empty, for each matched item, the locations of the erroneous bits are stored in $T$ and the components of $\overline{\mathbf{x}}$ with indices in $T$ will be flipped. The likelihood

of the revised vector $\hat{\mathbf{x}}_{j}$ with the channel output can be normalized as $-\mathbf{y}^{T} \hat{\mathbf{x}}_{j}$. This calculation is derived in [19], and its negative value is usually used as the objective function of the ML decoding model of LDPC codes as described in (2.7). The revised vector $\hat{\mathbf{x}}_{k}$ with the smallest $h_{k}=\mathbf{y}^{T} \hat{\mathbf{x}}_{k}$ has the largest likelihood with the channel output and will be output.

In order to deal with the multiple-matching problem, we use the ML criterion to select one of the matched items. It should be noted that codeword bits which are not in the trapping sets will not be flipped in any cases. Therefore, when calculating and comparing the likelihood, we only need to concern the union of all flipped bits of each revised vector, instead of all codeword bits. For simplicity, this union set can be relaxed to the set of all neighboring variable nodes of unsatisfied check nodes. So the negative normalized likelihood of a revised vector $\hat{\mathbf{x}}_{j}$ can be calculated as

$$
h_{j}=\sum_{i \in E} y_{i} \hat{x}_{j i},
$$

where $E=N_{c}\left(m_{1}\right) \cup \ldots \cup N_{c}\left(m_{p}\right)$ and $\left\{m_{1}, \ldots m_{p}\right\}$ is the set of unsatisfied check node indices.

\subsubsection{SPM Algorithm for General LDPC Codes}

We can see in Algorithm 6 that the most complex operation is the SPM algorithm in Line 8. The syndrome pattern $\mathbf{s}$ is an array. So the straight-forward matching algorithm needs to perform an array matching operation, which compares corresponding components of the two arrays one by one, over the entire list $\mathcal{L}_{1}$ to find all matching items. For a lexicographical order list, the comparison can be terminated in advance if a matching item is found and the next item is not matched with the target pattern. Here a list of vectors is said to be in lexicographical order if the corresponding components of the vectors in the list are non-decreasing from top to bottom. For the worst case, we need to perform $N$ array matching operations to find the matching item if the length of $\mathcal{L}_{1}$ is $N$. The complexity of this algorithm is then $\mathcal{O}\left(N b_{\max }\right)$, where $b_{\max }$ is the size of the array. 
The high complexity of the straight-forward SPM algorithm prevents applying our look-up table based syndrome decoder when the length of the look-up table is large. So we introduce a low complexity SPM algorithm that utilizes the power of the binary search algorithm. The binary search algorithm is an efficient algorithm to find a target value from a sorted list of numbers of length $N$. It starts a comparison from the middle of the list and halves the current searching space after each iteration. The average and worst-case complexity is $\log _{2}(N)$ [108]. In our case, items in $\mathcal{L}_{1}$ are all arrays, which makes it challenging to apply the binary search algorithm even if it is ordered. Therefore, a feature value key is extracted from each syndrome pattern in $\mathcal{L}_{1}$ in advance by a feature generation function

$$
k e y=G(\mathbf{s}),
$$

where $\mathbf{s}$ is the syndrome pattern. All feature values are stored in another list $\mathcal{L}_{0}$, and items in the look-up table are reordered based on the feature values. It should be noted the look-up table now includes three lists and the lengths of lists $\mathcal{L}_{0}, \mathcal{L}_{1}$ and $\mathcal{L}_{2}$ are all equal to the total number of trapping sets in the table. The feature generation function $G(\cdot)$ should generate as many unique feature values as possible from all syndrome patterns. Ideally, $G(\cdot)$ is an injective function (or called one-to-one function) which guarantees to generate different feature values for different syndrome patterns. As a result, the syndrome pattern can be directly located by its key value. In the SPM algorithm, the feature value of $\mathbf{s}$ is calculated first by $G(\cdot)$ which may not be an injective function. Then, instead of searching the array $\mathbf{s}$ in $\mathcal{L}_{1}$, this feature value is searched in $\mathcal{L}_{0}$. If some items are found to have the same feature value, the corresponding arrays in $\mathcal{L}_{1}$ will be further checked to make sure they are truly matched with the target pattern. The details of the SPM algorithm for general codes are as follows.

In Algorithm 7, the feature value of the target syndrome $\mathbf{s}$ is generated and stored in key. Then the binary search algorithm is applied on $\mathcal{L}_{0}$ to verify if this feature value is in $\mathcal{L}_{0}$. The binary search algorithm returns -1 if the feature value is not in $\mathcal{L}_{0}$. Otherwise, it returns the index of one matched feature value in $\mathcal{L}_{0}$. Set $M$ is used to store indices of all matching items. If the return value $i d$ is -1, the target syndrome pattern has no chance to be stored in the look-up table, and the output $M$ is an empty set. If $i d$ is not -1 , the subroutine in Line 4 will find the all other items which have the same feature value based on $i d$ and store all the indices in the set M. 


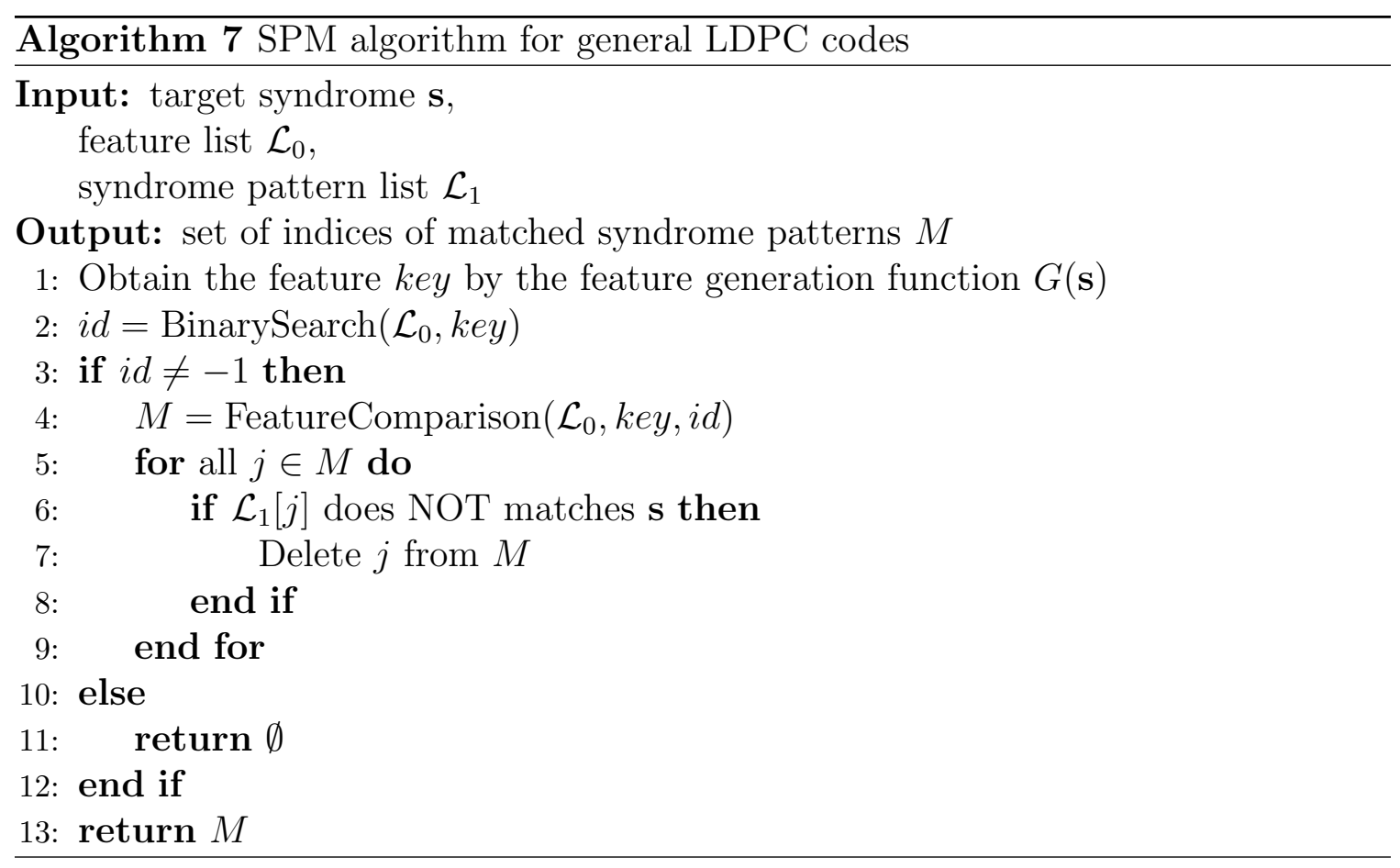

Since $\mathcal{L}_{0}$ is an ordered list, items with the same value must be in consecutive places. The subroutine will compare key with items near index id in $\mathcal{L}_{0}$, namely items with indices $i d-1, i d-2, \ldots$ and $i d+1, i d+2, \ldots$, and stop in either direction when the items are no longer key. However, not all syndrome patterns with indices in the set $M$ are matched with the target syndrome pattern. This is because the feature generation function $G(\cdot)$ may not be an injective function and different syndrome patterns may have the same feature value. Therefore, for each index in $M$, an array matching operation has to be used to further check if the corresponding syndrome patterns in $\mathcal{L}_{1}$ are the real matching items. If the syndrome pattern only has the same feature value but is not the same as $\mathbf{s}$, this index will be deleted from $M$.

By constructing list $\mathcal{L}_{0}$ via the feature generation function, we can get an approximation of the set of matching items. Only items with the same feature value as the target pattern are in the set and will be checked one by one by a complex array matching operation. It will be shown later this set can be located efficiently and the size of the set, denoted as $l e n_{\mathbf{s}}$, can be relatively small.

As explained before, the set can be efficiently found in two steps. Firstly, The feature value can be searched by a binary search algorithm whose average and the worst-case complexity is $\log _{2}(N)$. Secondly, If one item is matched, other items with the same feature value can be found easily since their indices must be consecutive. 
Therefore, for the worst case, we need only $\log _{2}(N)$ arithmetic comparisons to locate one possible matching item, a few more comparisons to find all other possible matching items, and then a few array matching operations to check further. The exact number of additional comparisons and array matching operations after the binary search depends on the number of trapping sets owning the same feature value. We will formally discuss the complexity and present some results later.

The size of this set is influenced by the trapping sets of the LDPC code itself and the feature generation function. If the LDPC code has many trapping sets with the same syndrome pattern, all of them will appear in the set. Apart from this, the feature generation function plays a critical role. In the ideal case, $G(\cdot)$ is injective and generates a unique feature for every different syndrome pattern. However, an injective function is impractical since $G(\cdot)$ will become extremely complex, and the range of the feature value will increase significantly. This will be explained again later, and we can give some practical implementations of the generation function. It will also be shown that even if $G(\cdot)$ is not a bijective function, this range is still quite small.

\subsubsection{SPM Algorithm for QC-LDPC Codes}

Algorithm 7 can directly be applied to QC-LDPC codes without utilizing their circulant structure. In this part, we first revisit the efficient representation of trapping sets of QC-LDPC codes introduced in Chapter 2 and propose an SPM algorithm for QC-LDPC codes.

For a QC-LDPC code with the $Q \times Q$ circulant sub-matrix, if one can find a single trapping set, there exist the other $Q-1$ trapping sets that can be generated by applying different quasi-cyclical shift amount $\Delta$ on this trapping set. Mathematically, if one $(a, b)$ trapping set is denoted by an error pattern $\left\{v_{1}, v_{2}, \ldots, v_{a}\right\}$ and a syndrome pattern $\left\{c_{1}, c_{2}, \ldots, c_{b}\right\}$, its isomorphic trapping set with QC-shift amount $\Delta$ can be obtained by (2.11) and (2.12), where $\left\lfloor\left(v_{i}-1\right) / Q\right\rfloor$ and $\left(v_{i}-1\right) \% Q$ are the module index and the circulant index of variable node $v_{i}$, respectively. Similarly, $\left\lfloor\left(c_{i}-1\right) / Q\right\rfloor$ and $\left(c_{i}-1\right) \% Q$ are the module index and the circulant index of variable node $v_{i}$, respectively. It can be found that the module index of any variable nodes or check nodes does not change no matter which shift amount $\Delta$ is applied. The MI vector $\left(\left\lfloor\left(c_{1}-1\right) / Q\right\rfloor,\left\lfloor\left(c_{2}-1\right) / Q\right\rfloor, \ldots,\left\lfloor\left(c_{b}-1\right) / Q\right\rfloor\right)$ is the common property of the whole isomorphism class. 
Example 3. Here is an example of the rate-5/6 $(648,540) Q C-L D P C$, denoted by $\mathcal{C}_{10}$, in the WiFi standard [\%]. The code $C_{10}$ has a circulant sub-matrix of $27 \times 27$. For a $(6,2)$ trapping set with the error pattern $\{41,158,568,587,596,619\}$ and the syndrome pattern $\{28,47\}$, there exist 26 other isomorphic trapping sets which can be generated by quasi-cyclically shifting the error pattern and the syndrome pattern. If the $Q C$-shift amount $\Delta=8$, we obtain an error pattern $\{49,139,576,568,604,600\}$ and the syndrome pattern $\{36,28\}$. We can find that the index of variable node $v_{568}$ is shifted to 576, and the index of variable node $v_{587}$ is shifted "back" to 568. For simplicity, the elements of the error pattern and the syndrome pattern are stored in the ascendant order as $\{49,139,568,576,600,604\}$, and $\{28,36\}$, respectively. The MI vector is $(1,1)$ and all trapping sets in this isomorphism class have the same MI vector.

The trapping sets representation method of QC-LDPC codes allows us to generate the other trapping sets in the same isomorphism class by applying different quasicyclic shifts to one trapping set. Therefore, it allows us to store only one trapping set per isomorphism class in the table, while still can generate all the other trapping sets in the same isomorphism class and then recover all failures caused by them. If the target syndrome pattern is found to be the QC-shift version of one item in $\mathcal{L}_{1}$ with QC-shift amount $\Delta$, which means they belong to the same isomorphism class, then its corresponding error pattern can be obtained by (2.11), and this error could be corrected. The procedure of justifying the QC-shift relationship between two syndrome patterns is simple when the lengths of the syndrome patterns stored in the table are 1. In this case, for the two syndrome patterns, if their indices of the only unsatisfied check node are the same, they are justified as being in the same isomorphism class, and $\Delta$ is the difference between the indices. However, for two syndrome patterns with more than one component, this justification and the calculation of $\Delta$ is not straight-forward. This is because indices of trapping sets are stored in the ascendant order in the look-up table and the relative positions of check nodes would change when the QC-shift operation is applied. In our Example 3 of $C_{10}$, the index of check node $c_{28}$ is shifted to 36 and the index of check node $c_{47}$ is shifted "back" to 28 when the shift amount $\Delta=8$. We cannot obtain $\Delta$ by simply calculating the difference between corresponding components of the two ordered index vectors $\{28,47\}$ and $\{28,36\}$. For the same reason, judging if one trapping set is the quasi-cyclic version of another is not simple. These two tasks become even more 
complicated when the length of the syndrome pattern is larger. To deal with these two difficulties, we define the concept of isomorphism sub-class of trapping sets.

Definition 1 (Isomorphic Sub-group). The isomorphism sub-classes of trapping sets is a partition of an isomorphism class of trapping sets. Inside each isomorphism subclass, any syndrome pattern of a member can be obtained by applying a linear shift on another member's syndrome pattern.

Here we divide one isomorphism class into several non-overlapping sub-classes. In each isomorphism sub-class, only linear shift operation is permitted to be applied to the syndrome pattern. If one member's syndrome pattern is the QC-shift version, but not the linear shift version, of another member's syndrome pattern, the two members belong to different sub-classes. Following this definition, two syndrome patterns can be easily distinguished as in the same sub-class or not by comparing the shifts between all corresponding components. If the shifts are all the same, they belong to the same sub-class, and this shift is $\Delta$. It should be noted that the MI vector is the same for all different sub-class since it is the common property of the whole group. It can also be found that, for all syndrome patterns in the same sub-class with different shift amounts of $\Delta$, the relative positions of all components keep the same, namely the differences between each component, and the last component are the same. These differences form a vector, $\left(c_{b}^{\Delta}-c_{1}^{\Delta}, c_{b}^{\Delta}-c_{2}^{\Delta}, \ldots, c_{b}^{\Delta}-c_{b-1}^{\Delta}\right)$, called the difference index vector of syndrome pattern of this isomorphism sub-class (or DI vector for short). For different isomorphism sub-classes, the DI vectors are different. It is the common property of members in the isomorphism sub-class.

Example 4. We also use $C_{10}$ and the same trapping sets as an instance. In Fig. 6.1, we list the syndrome patterns of these trapping sets and show the relationship between the isomorphism class and the sub-classes. The whole isomorphism class contains 27 members. They can be divided into two sub-classes. Sub-group 1 contains 8 members and sub-class 2 contains 19 members. The relative positions between check nodes keep the same for all members of the same sub-class, while the relative positions are different for different sub-classes. Syndrome pattern $\{28,36\}$ is a $Q C$-shift version of $\{28,47\}$, but not a linear shift version. Therefore, they are in the same isomorphism class but belong to different isomorphism sub-classes. The MI vector of these two sub-classes is all $(1,1)$. On the contrary, the DI vector for sub-class 1 is (19), and the DI vector for sub-class 2 is (8). The union of these two sub-classes is the complete isomorphism class. 


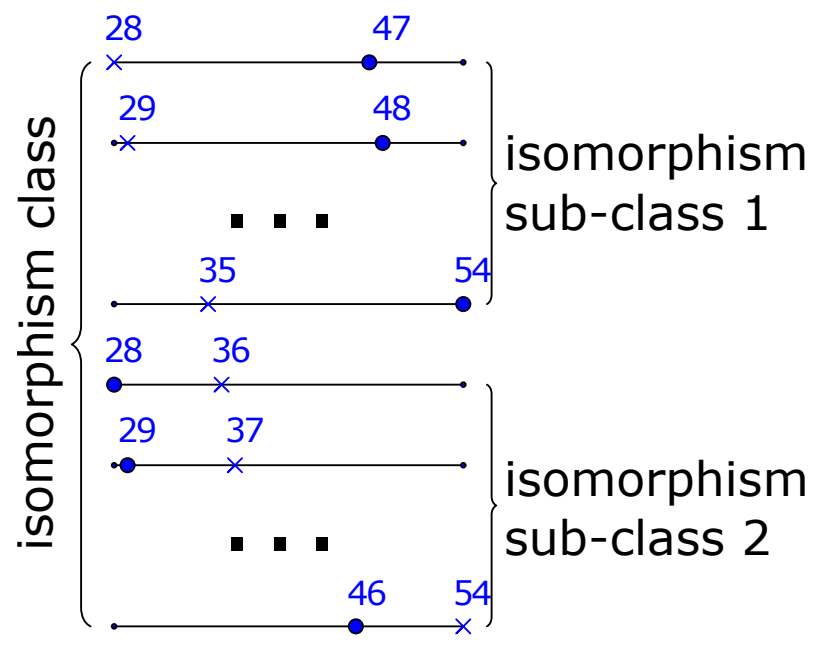

Figure 6.1: Illustration of the relationship between the isomorphism class and the isomorphism sub-class. Each line segment represents a syndrome pattern, and the numbers above are the indices. When the syndrome pattern $\{35,54\}$ is right-shifted again, check node $c_{54}$ is shifted to position 28 . The relative position between two check nodes changes, and the resulting syndrome pattern belongs to another sub-class.

Proposition 9. For a $Q C$ - $L D P C$ code with lifting degree $Q$ and $m$ check nodes, an $(a, b)$ isomorphism class of trapping sets can be divided into at least $\left\lceil b /\left(\frac{m}{Q}\right)\right\rceil$ and at most b isomorphism sub-classes.

Proof. For a QC-LDPC code with lifting degree $Q$ and maximum check node index $m$, the check node index range can be divided into $m / Q$ intervals as $[(i-1) * Q+1, i * Q]$, where $i=1,2, m / Q$. For the QC-shift operation on one syndrome pattern with $\Delta=1$, we increase all indices of the syndrome pattern by one. If some indices of the original syndrome pattern are at the upper boundaries of their intervals, the indices increased by one will be out of boundary and should be shifted "back" to the beginning of their intervals. Based on the definition of the isomorphism sub-class, this new syndrome pattern belongs to a new isomorphism sub-class. Therefore, the appearance of the shift-back during the QC-shift operation is corresponding to a new isomorphism subclass. The number of different isomorphism sub-classes is equal to the number of cases where different shift-backs happen when we apply QC-shift operations with different $\Delta$.

Let us start with any syndrome pattern in an $(a, b)$ isomorphism class and apply QC-shift operation with $\Delta=1$ to it for $Q-1$ times consecutively. During this procedure, all $b$ check node indices will be shifted back once, but some indices may be 
shifted back together. If there is at most one check node index is out of boundary and must be shifted back each time, there will be $b$ different shift-back cases happening. Then there are $b$ isomorphism sub-classes. This happens when the circulant indices of all components of the syndrome pattern are different. If several check nodes share the same circulant index, their indices will be on their upper boundaries at the same time and will be shifted back together. Since there are totally $m / Q$ intervals, there will be at most $m / Q$ check node indices being on their boundaries simultaneously. As a result, there are at least $\left\lceil b /\left(\frac{m}{Q}\right)\right\rceil$ shift-back happen, corresponding to at least $\left\lceil b /\left(\frac{m}{Q}\right)\right\rceil$ isomorphism sub-classes.

Here we propose an SPM algorithm for QC-LDPC codes which only stores a portion of trapping sets in the look-up table while being equivalent to saving all trapping sets of the same class. The trapping set leading to the error can be efficiently located if one of its isomorphic members is in the look-up table. In order to combine the advantages of the binary search and the isomorphism sub-class, we need to find a common property shared by every member of the isomorphism sub-class and devise a feature generation function based on this property. Thus, syndrome patterns in the same isomorphism sub-class will have the same feature value. We can locate one or several trapping set sub-classes that the target trapping set may belong to first. Then we can further check if the target syndrome pattern truly belongs to these trapping set sub-classes. Lastly, we can regenerate the corresponding error pattern information and revise the hard decision result of the previous stage decoder. Here we use the isomorphism sub-class, which means storing one member per one sub-class in the table. We know that the MI vector and the DI vector are the same for all members in a sub-class. Therefore, we can devise a feature generation function based on these two entities. Again, the feature generation function used here should generate as many different features as possible.

The framework of the SPM algorithm for QC-LDPC codes is similar to Algorithm 7. The only difference is in Line 6 where we try to further compare $\mathcal{L}_{1}[j]$ with $\mathbf{s}$. For QC-LDPC codes, the target pattern $\mathbf{s}$ is not required to be the same as the items in $\mathcal{L}_{1}$. This decision condition in Line 6 should be replaced by judging if the two syndrome patterns are in the same isomorphism sub-class. This operation is called the trapping set sub-class justification and is shown in Algorithm 8. Inside the Algorithm, we first judge if the stored item in $\mathcal{L}_{1}$ is a linear shift version of the target 
pattern. Then we verify that the two patterns also have the same MI vectors.

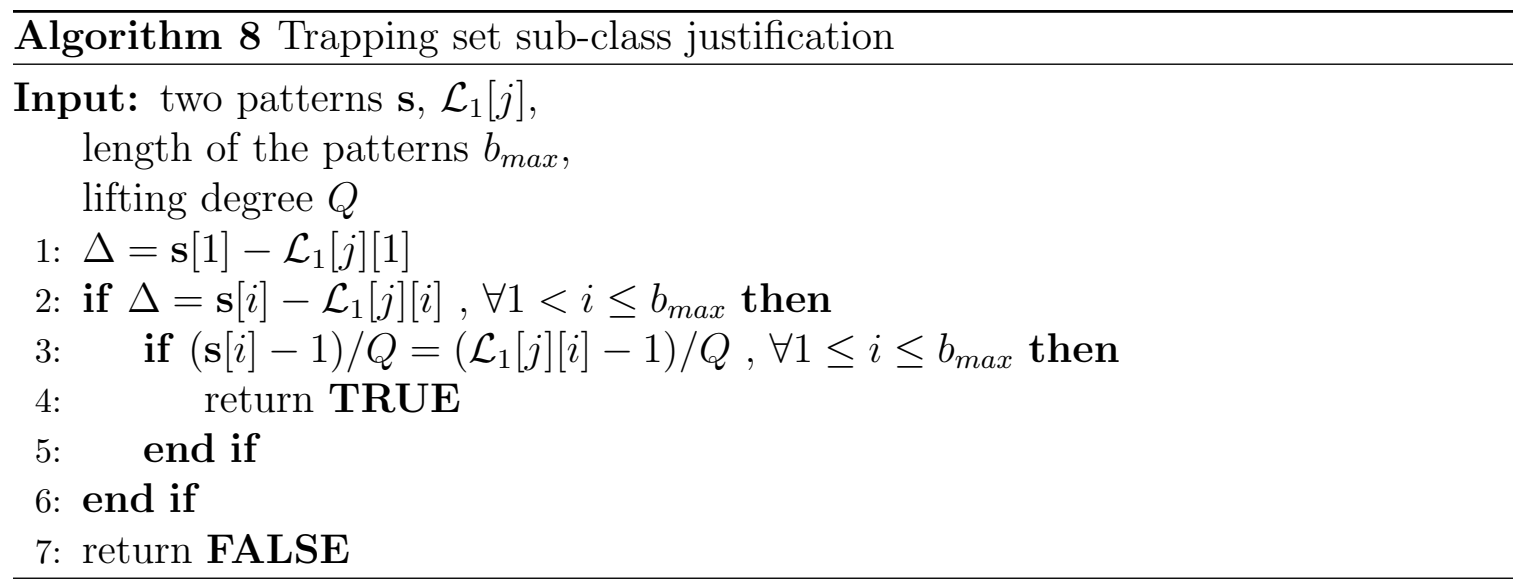

The Algorithm 6 should also be modified for QC-LDPC codes since we cannot directly get the corresponding error pattern information $T$ in Line 13 by the index. For QC-LDPC codes, the set $T$ can be obtained by applying the QC-shift operation with amount $\Delta$ to the error patterns $\mathcal{L}_{2}[j]$ according to (2.12). Therefore, Line 13 is replaced by the following operations:

1: $\Delta=\mathbf{s}[1]-\mathcal{L}_{1}[j][1]$

2: $T_{i} \leftarrow$ Apply QC-shift on non-zero part of $\mathcal{L}_{2}[j]$ with amount $\Delta$

\subsubsection{Construction of the Look-Up Table}

The construction of a look-up table includes four steps: obtaining the dominant trapping sets, formatting the trapping sets, generating the feature value list $\mathcal{L}_{0}$, and reordering the look-up table.

In the first step, the Monte Carlo simulation can be performed in advance to decide the dominant trapping set classes, and trapping sets belong to these classes should be searched. In order to correct all failures caused by a specific trapping set class, the look-up table has to store all members of this trapping set class (except for QC-LDPC codes). Most methods to find the trapping sets, like [82, 84, 94, 95], can only give a partial list of a trapping set class. The IP model in [96] can output the exhaustive list of a trapping set class, but it cannot be applied to long-length codes. Therefore, we choose to use the method proposed in [97] which can find all leafless elementary trapping sets smaller than size $\left(a_{\max }, b_{\max }\right)$. 
Since the sizes of the trapping sets are different, we have to modify trapping sets to make them all have the same size in the second step. Let $a_{\max }$ and $b_{\max }$ denote the maximum lengths of the error patterns and the syndrome patterns, respectively. For a trapping set $(a, b)$, we can append $a_{\max }-a$ and $b_{\max }-b 0$ s at the end of the error pattern and the syndrome pattern, respectively. It should be noted that the non-zero components of the error patterns and the syndrome patterns are in non-decreasing order, and all zeros are placed after the largest non-zero component.

After that, a feature generation should be selected and used to construct $\mathcal{L}_{0}$. Ideally, $G(\cdot)$ should be an injective function, which means for different inputs, the outputs are different. In this case, if we have located the range of trapping sets that have the same feature value as the target syndrome pattern, we do not have to call any array matching operations to check further. However, the injective function is not a practical choice of the feature generation function because it is not only too complex for our scenario but also too costly to deploy. For example, secure hash algorithms (SHA) [109] are a family of hash functions that can be regarded as injective functions because the probability that two different inputs have the same hash value, namely the collision probability, is so small. ${ }^{1}$ Nonetheless, the SHA are iterative, which leads to a long time delay. Moreover, it will consume huge space to store $\mathcal{L}_{0}$ since the hash value generated by SHA has a long width. The long time delay and large memory requirement of SHA are acceptable for security-sensitive applications but do not conform to our low-complexity requirement. We need to consider a balance between efficiency and cost, and find a simple function which can generate as many different feature values as possible and keep the range of the feature as small as possible. In the last step, all trapping sets $\left(\mathcal{L}_{1}\right.$ and $\left.\mathcal{L}_{2}\right)$ are stored in the look-up table together with $\mathcal{L}_{0}$, and the rows are reordered based on the elements of $\mathcal{L}_{0}$.

Here we propose the following four possible feature generation functions for general

\footnotetext{
${ }^{1}$ For the SHA3-224n algorithm [110], one of the latest members of the SHA family, any input will result in a 224 bits hash value. The collision probability is around $1.9 \times 10^{-56}$ when we have 1 million different inputs. For less number of inputs, this probability is even smaller.
} 
LDPC codes:

$$
\begin{aligned}
G_{1}(\mathbf{s}) & =\sum_{j=1}^{b_{\max }} s_{j}, \\
G_{2}(\mathbf{s}) & =\sum_{j=1}^{b_{\max }} s_{j} * 10^{j-1}, \\
G_{3}(\mathbf{s}) & =\sum_{j=1}^{b_{\max }} s_{j}^{2}, \\
G_{4}(\mathbf{s}) & =\sum_{j=1}^{b_{\max }} s_{j}^{j} .
\end{aligned}
$$

The four functions are not iterative and involve simple arithmetic operations. For a syndrome pattern $\mathbf{s}$ of length $b_{\max }, G_{1}(\mathbf{s})$ sums all its components and $G_{3}(\mathbf{s})$ sums the square values of the components. In the other two functions, the components at different positions have different weights or operations. For two syndrome patterns $\{2,3\}$ and $\{1,4\}$, if we use $G_{1}(\mathbf{s})$ to generate the features, the features are all equal to 5 . If we use $G_{2}(\mathbf{s})$, the features will be 32 and 41, respectively. For two syndrome patterns $\{1,101\}$ and $\{11,100\}, G_{2}(\mathbf{s})$ will generate the same feature 1011 , but the $G_{1}(\mathbf{s})$ and $G_{3}(\mathbf{s})$ will output different features for the two different syndrome pattern. It should be noted zeros may be appended at the end of the syndrome pattern, but they do not affect the feature values calculated by these four functions. For the simpler implementation, the component $10^{j-1}$ in (6.3) might be replaced by $2^{3(j-1)}$.

To evaluate feature generation functions for a given LDPC code, we construct a look-up table including all trapping sets we are interested in and the generated feature value list $\mathcal{L}_{0}$, and then check for how many cases there will be a collision. We note that the feature generation function should introduce as few collisions as possible, but even if there are collisions, it can be solved by further checking the actual syndrome pattern. We construct two look-up tables for a $(4095,3358)$ random code, denoted by $\mathcal{C}_{9}$, picked up from the website [111]. The first look-up table (LUT1) contains all 1819 trapping sets of three classes, $(5,1),(6,2)$, and $(7,1)$ classes. We find that 1801 of them have a unique syndrome pattern, which means the remaining 18 trapping sets will have the same feature values as others no matter which feature generation function is used. For example, there exist two $(5,1)$ trapping sets and one $(7,1)$ trapping set with the same syndrome pattern $\{1\}$. The second look-up table (LUT2) contains all trapping sets belonging to 6 different classes $(4,2),(5,1),(6,2)(7,1),(8,2)$, and $(9,1)$. There is a total of 16212 trapping sets, and 15524 of them have a unique syndrome pattern. Table 6.1 shows the number of unique features and the range of the features 


\begin{tabular}{|l||c|l||c|l|}
\hline & $\begin{array}{l}\text { number of unique } \\
\text { features in LUT1 }\end{array}$ & $\begin{array}{l}\text { range of features } \\
\text { in LUT1 }\end{array}$ & $\begin{array}{l}\text { number of unique } \\
\text { features in LUT2 }\end{array}$ & $\begin{array}{l}\text { range of features } \\
\text { in LUT2 }\end{array}$ \\
\hline$G_{1}(\mathbf{x})$ & 942 & {$[6,1442]$} & 1420 & {$[3,1465]$} \\
\hline$G_{2}(\mathbf{x})$ & 1589 & {$[6,8041]$} & 6410 & {$[3,8096]$} \\
\hline$G_{3}(\mathbf{x})$ & 1793 & {$[36,1039684]$} & 14992 & {$[9,1073113]$} \\
\hline$G_{4}(\mathbf{x})$ & 1801 & {$[6,543840]$} & 15518 & {$[3,543895]$} \\
\hline
\end{tabular}

Table 6.1: Comparison of different feature generation functions for the random LDPC code $\mathcal{C}_{9}$. LUT1 includes totally 1819 trapping sets and 1801 of them have a unique syndrome pattern. LUT2 includes totally 16212 trapping sets and 15524 of them have a unique syndrome pattern.

generated by four feature generation functions for the two look-up tables. It can be seen that $G_{1}(\mathbf{x})$ and $G_{2}(\mathbf{x})$ produce less unique features, but the range of the features are quite small. Almost every different trapping set (syndrome pattern) has a unique feature if we use $G_{3}(\mathbf{x})$ or $G_{4}(\mathbf{x})$, which means we hardly need to perform further check if we have found a matched feature value. However, the range of the feature values grows significantly, especially for $G_{3}(\mathbf{x})$, which means more bits are required to represent the larger value. We cannot guarantee $G_{4}(\mathbf{x})$ is the best feature generation function, but we try to show that a good feature generation function should generate as many unique feature values as possible while keeping the range of feature values as small as possible. Obviously, there is a trade-off between efficiency and cost. We note that some existing hash functions are also suitable for our application. For example, the folding function breaks the input into segments and calculates the XOR result of all segments. This is quite similar to our proposed $G_{1}(\mathbf{x})$. More examples can be found in [108].

For a QC-LDPC code with lifting degree $Q$, we select one member for each isomorphism sub-class and store them in the look-up table. For the sub-class with a syndrome pattern $\mathbf{s}=\left(s_{1}, s_{2}, \ldots, s_{d}, 0, \ldots, 0\right)$, the MI vector $\left(\left\lfloor\left(s_{1}-1\right) / Q\right\rfloor,\left\lfloor\left(s_{2}-\right.\right.\right.$ $\left.1) / Q\rfloor, \ldots,\left\lfloor\left(s_{d}-1\right) / Q\right\rfloor\right)$ and the DI vector $\left(s_{d}-s_{1}, s_{d}-s_{2}, \ldots, s_{d}-s_{d-1}\right)$ are the common properties for all its members. The index $d$ is the index of the last non-zero component of the syndrome pattern s. The possible zero part cannot be involved in the calculation of the MI vector and the DI vector. Here are four possible feature generation functions for QC-LDPC codes based on the common properties: 


$$
\begin{aligned}
G_{1}^{\prime}(\mathbf{s}) & =\sum_{j=1}^{d}\left\lfloor\left(s_{j}-1\right) / Q\right\rfloor, \\
G_{2}^{\prime}(\mathbf{s}) & =\sum_{j=1}^{d}\left\lfloor\left(s_{j}-1\right) / Q\right\rfloor * 10^{j-1}, \\
G_{3}^{\prime}(\mathbf{s}) & =\sum_{j=1}^{d}\left(s_{d}-s_{j}\right) * 10^{j-1}+\left(\left\lfloor\left(s_{j}-1\right) / Q\right\rfloor\right) * 100^{j-1}, \\
G_{4}^{\prime}(\mathbf{s}) & =\sum_{j=1}^{d}\left(s_{d}-s_{j}\right) * 10^{j-1}+\left(\left\lfloor\left(s_{j}-1\right) / Q\right\rfloor\right) * 100^{j} .
\end{aligned}
$$

They are also not iterative and involves only simple arithmetic operations. For a syndrome pattern s with $d$ non-zero elements, $G_{1}^{\prime}(\mathbf{s})$ sums all components of the MI vector. $G_{2}^{\prime}(\mathbf{s})$ is the weighted version of $G_{1}^{\prime}(\mathbf{s})$. In the other two cases, both the DI vector and the MI vector are taken into account with different weights. It should be emphasized here that the calculation of the feature value only involves non-zero elements (real unsatisfied check nodes) of the syndrome pattern. Also, for the simpler implementation, the component $10^{j-1}$ in (6.7) might be replaced by $2^{3(j-1)}$ and the component $100^{j-1}$ in (6.8) might be replaced by $2^{7(j-1)}$.

We also construct two look-up tables for $C_{10}$, and evaluate the collision probability of the four functions. The first look-up table (LUT1) represents 297 trapping sets. 81 of them belong to the $(5,1)$ class, and the other 216 trapping sets belong to the $(6,1)$ class. We select one member for each isomorphism sub-class and get 11 representatives, 3 from the $(5,1)$ class and 8 from the $(6,1)$ class. All of them have different syndrome patterns, but some have the same MI vector and the DI vector. There are totally 4 different combinations of the MI vector and the DI vector, which means there are at most 4 different feature values generated by the four functions. The second look-up table (LUT2) represents all trapping sets belonging to 9 different classes, $(4,1),(4,2),(5,1),(5,2),(6,1),(6,2),(7,1),(7,2)$, and $(8,1)$ classes. There are a total of 29484 trapping sets, and 2097 of them are stored in the look-up table. Among them, 1277 trapping sets have different syndrome patterns, and there are 422 different combinations of the MI vector and the DI vector. Table 6.2 shows the number of unique features and the range of the features generated by the four feature generation functions for the two look-up tables. It can be seen that for LUT1 which contains only length 1 syndrome patterns, these functions all generate 4 different feature values. This is because the DI vector does not exist, or we may assume it as 0 for all trapping sets. The MI vector is the only property we can use, and it only 


\begin{tabular}{|l||c|l||c|l|}
\hline & $\begin{array}{l}\text { number of unique } \\
\text { features in LUT1 }\end{array}$ & $\begin{array}{l}\text { range of features } \\
\text { in LUT1 }\end{array}$ & $\begin{array}{l}\text { number of unique } \\
\text { features in LUT2 }\end{array}$ & $\begin{array}{l}\text { range of features } \\
\text { in LUT2 }\end{array}$ \\
\hline$G_{1}^{\prime}(\mathbf{x})$ & 4 & {$[0,3]$} & 7 & {$[0,6]$} \\
\hline$G_{2}^{\prime}(\mathbf{x})$ & 4 & {$[0,3]$} & 13 & {$[0,33]$} \\
\hline$G_{3}^{\prime}(\mathbf{x})$ & 4 & {$[0,3]$} & 263 & {$[0,407]$} \\
\hline$G_{4}^{\prime}(\mathbf{x})$ & 4 & {$[0,300]$} & 422 & {$[0,30326]$} \\
\hline
\end{tabular}

Table 6.2: Comparison of different feature generation function for the QC-LDPC code $C_{10}$. LUT1 includes totally 11 trapping sets and 4 different combinations of the MI vector and the DI vector. LUT2 includes totally 2097 trapping sets and 422 different combinations of the MI vector and the DI vector.

has one component. In addition, the number of check nodes for $C_{10}$ is $108=27 * 4$, which means the module index in the MI vector can only be $1,2,3$, or 4 . In the case of LUT2, $G_{2}^{\prime}(\mathbf{x})$ generates more unique feature values than $G_{1}^{\prime}(\mathbf{x})$, but this number is still relatively small compared with the number of different combinations of the MI vector and the DI vector. $G_{3}^{\prime}(\mathbf{x})$ and $G_{4}^{\prime}(\mathbf{x})$ can generate more unique feature values because the DI vector is also considered in the calculation and assigned a large weight. The module index for $C_{10}$ can be easily "drowned" by the weighted sum of DI vector's components since it is a non-negative integer at most 4 . Therefore, $G_{4}^{\prime}(\mathbf{x})$ assigns the module index a larger weight and generates the largest number of unique features. At the same time, the range of the feature values of $G_{4}^{\prime}(\mathbf{x})$ is also increased.

\subsection{Complexity Analysis}

The complexity of our enhanced look-up table based syndrome decoder depends mostly on the SPM algorithm. Compared with the straight-forward SPM algorithm, our proposed SPM algorithms require an extra list $\mathcal{L}_{0}$. The additional memory consumption results in much less computation. Here we discuss the computational complexity and the storage complexity based on the feature generation function. We also suggest several variations of the SPM algorithm to provide more freedom of complexity trade-off.

As we stated before, for an ordered list of length $N$, the straight-forward SPM algorithm requires $N$ array matching operations to find the matching item in the worst case. The complexity of this algorithm is then $\mathcal{O}\left(N b_{\max }\right)$, where $b_{\max }$ is the length of the array. Our proposed SPM algorithm for general LDPC codes is based 
on the binary search algorithm and requires at $\operatorname{most} \log _{2}(N)$ arithmetic comparisons to locate one possible matching item. If the number of unique features generated by a feature generation function is $M$, the average number of syndrome patterns sharing the same feature value is $N / M$. Therefore, on average, there exist other $N / M-1$ items having the same feature value. $N / M+1$ more arithmetic comparisons should be performed forward or backward to find all matched items, and it stops when it finds two values not the same with them. Later the algorithm needs $N / M$ more array matching operations to further check if they are the real matching syndrome pattern.

For a QC-LDPC code with $N^{\prime}$ trapping sets stored in the table and $M^{\prime}$ unique features generated from $N^{\prime}$ syndrome patterns, the specialized SPM algorithm for QC-LDPC codes also requires at most $\log _{2}\left(N^{\prime}\right)$ arithmetic comparisons to locate one possible matching item, and $N^{\prime} / M^{\prime}+1$ more arithmetic comparisons to find all matching features. It later needs $N^{\prime} / M^{\prime}$ more trapping set sub-class justification operations to further check if they belong to the same sub-class with the target syndrome pattern.

The calculation of the feature value also introduces additional computational complexity. For example, for $G_{2}(\mathbf{x})$ for general LDPC codes, we need to perform $b_{\max }$ multiplication operations and $b_{\max }-1$ sum operation, but we note that the number of unsatisfied check nodes $b$ is usually less than 4 , and only the feature value of the target syndrome pattern should be calculated. So this computation can be neglected. We note that the SPM algorithm returns the set $M$ which contains indices of all matched syndrome patterns. The indices can be directed used to retrieve the corresponding error patterns for general LDPC codes. For QC-LDPC codes, the QC-shift operation should be performed to obtain the error pattern information.

Apart from the storage for the trapping sets themselves, our proposed SPM algorithms require the storage for the feature list $\mathcal{L}_{0}$. The extra storage for list $\mathcal{L}_{0}$ depends mainly on the range of the feature value. For an $(n, k)$ LDPC code with $m$ check nodes, if the maximum size of trapping sets we would like to add in the table is $\left(a_{\max }, b_{\max }\right)$, storing $N$ trapping sets requires $\left(\left\lceil\log _{2}(n)\right\rceil a_{\max }+\left\lceil\log _{2}(m)\right\rceil b_{\max }\right) N$ bits. If the maximum feature generated is keymax , we need additional $\left\lceil\log _{2}\left(k e y_{\max }\right)\right\rceil N$ bits to construct list $\mathcal{L}_{0}$. The additional storage ratio is

$$
\frac{\left\lceil\log _{2}\left(k e y_{\max }\right)\right\rceil}{\left\lceil\log _{2}(n)\right\rceil a_{\max }+\left\lceil\log _{2}(m)\right\rceil b_{\max }} \approx \log _{\left(n^{a} \max m^{b_{\max }}\right)}\left(k e y_{\max }\right)
$$


For QC-LDPC codes, if the SPM algorithm for QC-LDPC codes is used, we do not need to store all $N$ trapping sets to correct the failures caused by them. The number of trapping sets stored in the look-up table can be reduced to the number of isomorphism sub-classes, and it is usually a ratio of $N$. We give the following proposition to show the lower bound and the upper bound of the ratio $r$.

Proposition 10. For a $Q C$ - $L D P C$ code with lifting degree $Q$ and $m$ check nodes, if the total number of its $(a, b)$ trapping sets is $N$, the proposed look-up table based decoder combined with the SPM algorithm for QC-LDPC codes can correct all failures caused by these trapping sets by storing only $r * N$ trapping sets, where $\frac{\left\lceil b /\left(\frac{m}{Q}\right)\right\rceil}{Q} \leq r \leq \frac{b}{Q}$.

Proof. An $(a, b)$ trapping set class may include several different isomorphism classes. It means two trapping sets may have the same size $a$ and $b$, but belong to different isomorphism classes. Each isomorphism class includes $Q$ trapping sets for the QCLDPC code with lifting degree $Q$. Then the number of different $(a, b)$ isomorphism classes is equal to $\frac{N}{Q}$. From Proposition 9, each $(a, b)$ isomorphism class can be divided into at least $\left\lceil b /\left(\frac{m}{Q}\right)\right\rceil$ and at most $b$ sub-classes. Therefore, we only need to store at least $\left\lceil b /\left(\frac{m}{Q}\right)\right\rceil \frac{N}{Q}$ and at most $b \frac{N}{Q}$ trapping sets. Thus the storage ratio satisfies $\frac{\left\lceil b /\left(\frac{m}{Q}\right)\right\rceil}{Q} \leq r \leq \frac{b}{Q}$.

Generally, if the SPM algorithm for QC-LDPC codes is employed, $(1-r) * 100 \%$ storage can be saved than when we still regard the QC-LDPC code as a random code. From the upper bound of $r$, we conclude that more than $\left(1-\frac{b}{Q}\right) * 100 \%$ storage can be saved. Specifically, for $b=2$, we can save more than $\left(1-\frac{2}{Q}\right) * 100 \%$ memory.

Corollary 4. For a $Q C$-LDPC code with lifting degree $Q$, if the total number of its $(a, 1)$ trapping sets is $N$, the proposed look-up table based decoder combined with the SPM algorithm for QC-LDPC codes can correct all failures caused by these trapping sets by storing only $\frac{1}{Q} N$ of them.

This corollary is for the case where $b=1$. It holds because the upper bound and the lower bound all equal to $\frac{1}{Q}$. It also matches with the result we get before for $\mathcal{C}_{10}$ whose lifting degree is 27.81 trapping sets of the $(5,1)$ class are represented by 3 of them, and 216 trapping sets of the $(6,1)$ class are represented by 8 of them.

\footnotetext{
${ }^{2}$ This is an upper bound of the total comparison involved in the variation 3.

${ }^{3}$ The comparison operation here is much more complex than that of the others because the number of bits to represent the whole syndrome pattern is usually much larger than that of the maximum feature value.
} 


\begin{tabular}{|l|c|c|c|c|c|}
\hline & Total Storage (bit) & Comparison & $\begin{array}{c}\text { Feature } \\
\text { Calculation }\end{array}$ & $\begin{array}{c}\text { Array } \\
\text { Matching }\end{array}$ & $\begin{array}{c}\text { Sub-group } \\
\text { Justification }\end{array}$ \\
\hline $\begin{array}{l}\text { straight forward } \\
\text { algorithm in [82 }\end{array}$ & $\begin{array}{c}\left(\left\lceil\log _{2}(n)\right\rceil a+\right. \\
\left.\left\lceil\log _{2}(m)\right\rceil b\right) N\end{array}$ & 0 & 0 & $\mathrm{~N}$ & 0 \\
\hline $\begin{array}{l}\text { proposed algorithm } \\
\text { (general LDPC) }\end{array}$ & $\begin{array}{c}\left(\left\lceil\log _{2}(n)\right\rceil a+\left\lceil\log _{2}(m)\right\rceil b+\right. \\
\left.\left\lceil\log _{2}(k e y)\right\rceil\right) N\end{array}$ & $\log _{2}(N)+N / M+1$ & 1 & $N / M$ & 0 \\
\hline $\begin{array}{l}\text { proposed algorithm } \\
\text { (QC-LDPC) }\end{array}$ & $\begin{array}{c}\left(\left\lceil\log _{2}(n)\right\rceil a+\left\lceil\log _{2}(m)\right\rceil b+\right. \\
\left.\left\lceil\log _{2}(k e y)\right\rceil\right) N^{\prime}\end{array}$ & $\begin{array}{c}\log _{2}\left(N^{\prime}\right)+ \\
N^{\prime} / M^{\prime}+1\end{array}$ & 1 & 0 & $N^{\prime} / M^{\prime}$ \\
\hline $\begin{array}{l}\text { Variation 1 } \\
\text { (general LDPC) }\end{array}$ & $\begin{array}{c}\left(\left\lceil\log _{2}(n)\right\rceil a+\right. \\
\left.\left\lceil\log _{2}(m)\right\rceil b\right) N\end{array}$ & $\log _{2}(N)+N / M+1$ & $\log _{2}(N)+1$ & $N / M$ & 0 \\
\hline $\begin{array}{l}\text { Variation 2 } \\
\text { (QC-LDPC) }\end{array}$ & $\begin{array}{c}\left(\left\lceil\log _{2}(n)\right\rceil a+\right. \\
\left.\left\lceil\log _{2}(m)\right\rceil b\right) N^{\prime}\end{array}$ & $\begin{array}{c}\log _{2}\left(N^{\prime}\right)+ \\
N^{\prime} / M^{\prime}+1\end{array}$ & $\log _{2}\left(N^{\prime}\right)+1$ & 0 & $N^{\prime} / M^{\prime}$ \\
\hline $\begin{array}{l}\text { Variation 3 } \\
\text { general LDPC) }\end{array}$ & $\begin{array}{l}\left(\left\lceil\log _{2}(n)\right\rceil a+\right. \\
\left.\left\lceil\log _{2}(m)\right\rceil b\right) N\end{array}$ & $\left(\log _{2}(N) b+N b^{2}\right.$ & 0 & 0 & 0 \\
\hline $\begin{array}{l}\text { Variation 4 } \\
\text { general LDPC) }\end{array}$ & $\begin{array}{l}\left(\left\lceil\log _{2}(n)\right\rceil a+\right. \\
\left.\left\lceil\log _{2}(m)\right\rceil b\right) N\end{array}$ & $\log _{2}(N)^{3}$ & 0 & 0 & 0 \\
\hline
\end{tabular}

Table 6.3: Complexity comparison of the total consumed storage and the average numbers of different operations for the proposed SPM algorithms and their variations. For simplicity, the subscriptions of $a_{\max }, b_{\max }$ and $k e y_{\max }$ are dropped. The QC-shift operations out of the SPM algorithm for QC-LDPC codes are not listed.

To meet different implementation requirements, we propose four variations of the SPM algorithms. If we calculate the feature values during the decoding, we can save the storage for $\mathcal{L}_{0}$. This idea leads to variation 1 and variation 2 for general LDPC codes and QC-LDPC codes, respectively. It is also possible if we apply binary search operations on the corresponding components of the syndrome patterns for $b_{\max }$ times. After the first binary search operation on the first components of the syndrome patterns, we have to find the set of syndrome patterns preserving the same first component. Then the second binary search is applied to this set. The procedure stops when all components are compared. This is variation 3 which saves the storage for $\mathcal{L}_{0}$, the calculation of feature values, and the array matching operations, but introduces more comparisons. The number of comparisons includes the comparison operations in $b_{\max }$ binary search procedure and $b_{\max }$ procedures for refining the set of matched items. The exact number depends on the target syndrome pattern and the look-up table. It is even possible that we consider the whole syndrome pattern as a binary sequence and apply the binary search algorithm to it by regarding it as a single extra-large number. This is variation 4 which requires the least storage and computation. However, it has a higher hardware complexity because of the long width comparison. It should be noted that the ideas of variation 3 and variation 4 
cannot take advantage of the efficient representation of QC-LDPC code. So only the proposed syndrome pattern algorithm for QC-LDPC codes and its variation 2 do not have to store all the trapping set in the look-up table. The complexity information is summarized in Table 6.3.

\subsection{Simulation Results}

In this section, we will construct several look-up tables and present the performance of our proposed look-up table based syndrome decoder for the $(4095,3358)$ random code $\mathcal{C}_{9}$ and the $(648,540)$ QC-LDPC code $\mathcal{C}_{10}$ we used earlier.

The simulations are performed over the AWGN channel with BPSK. Quantized MS decoder [12] with 5 bits quantization is adopted as the first stage decoder. The clipping threshold is 1.8 for $\mathcal{C}_{9}$ and 1.25 for $\mathcal{C}_{10}$. The maximum iteration is 100 . We use the quantized MS decoder because the soft-decision belief propagation decoder often converges to a union of multiple small trapping sets, instead of single small trapping set as stated in [96].

\subsubsection{General LDPC code}

For the random code $\mathcal{C}_{9}, 9000$ decoding failures are collected for the quantized MS decode. The corresponding channel output vectors are also recorded. We analyze all decoding failures at $E_{b} / N_{0}=4.4 \mathrm{~dB}$ and find about $90 \%$ of them are caused by three classes of trapping sets, $(5,1),(6,2)$, and $(7,1)$ classes. Therefore, we construct the first look-up table (LUT1) which contains all trapping sets of these three classes, and the total number is 1819. The second look-up table (LUT2) we constructed contains not only these three classes but also the next dominant classes, $(4,2),(8,2)$, and $(9,1)$ classes. The total number of trapping sets obtained by the exhaustive trapping set search algorithm is 16212. These two tables are also used as examples in Section 6.2.4.

Based on our previous computational complexity analysis, if we use LUT2, in the worst case, the straight-forward SPM algorithm requires 16212 array matching operations. In contrast, we only need at most $\left\lceil\log _{2}(16212)\right\rceil=14$ arithmetic comparisons to locate the matching feature. If $G_{2}(\mathbf{x})$ is used to generate features, the average number of trapping sets that share the same feature is about 3 because the 


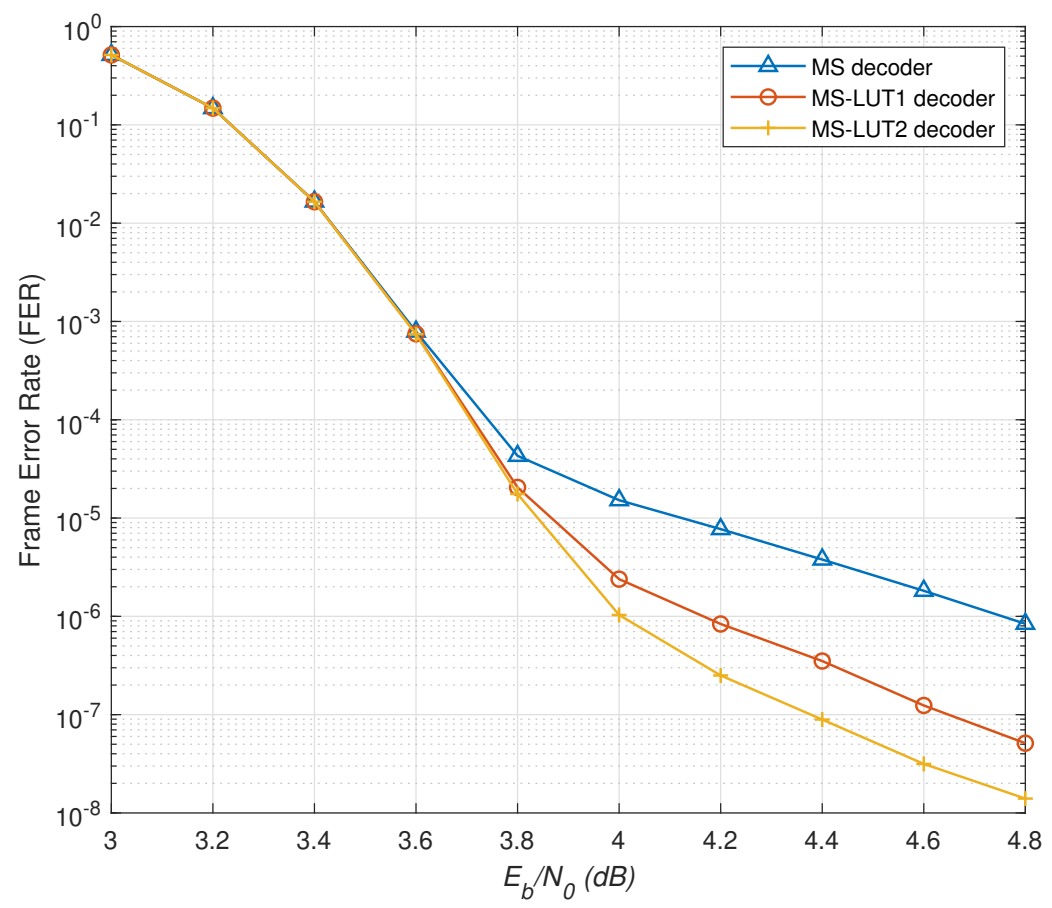

Figure 6.2: FER performances of $\mathcal{C}_{9}$ under the quantized MS decoder and the proposed look-up table based syndrome decoder with different look-up tables.

number of unique features is 6410 . Then 4 more arithmetic comparisons and 3 array matching operations will be performed later on average. Indeed, in LUT2, there are at most 11 trapping sets sharing the same feature. Therefore, we at most need 12 more arithmetic comparisons and 11 array matching operations after the binary search operation. The maximum value of the feature is 8096 . Thus we need 13 bits to represent the features and the whole feature list $\mathcal{L}_{0}$ would cost about 26.3 KByte. The total memory requirement for $\mathcal{L}_{1}$ and $\mathcal{L}_{2}$ is 259.4 KByte, which means storing $\mathcal{L}_{0}$ takes $10.2 \%$ more space than only storing lists $\mathcal{L}_{1}$ and $\mathcal{L}_{2}$. If we use $G_{4}(\mathbf{x})$ to generate features, almost every different syndrome patterns have different features, and there are at most 5 trapping sets sharing the same feature. Then we only need to perform 3 more arithmetic comparisons and 2 array matching operations on average or 6 arithmetic comparisons and 5 array matching operations in the worst case. Consequently, we need 20 bits to represent the features and the whole feature list $\mathcal{L}_{0}$ would cost $40.5 \mathrm{KByte}$, consuming $15.6 \%$ more space. For the balance between efficiency and cost, the $G_{2}(\mathbf{x})$ is used inside the SPM algorithm.

Fig. 6.2 shows the performances of $\mathcal{C}_{9}$ under the quantized MS decoder and the 
proposed look-up table based syndrome decoder with LUT1 and LUT2. It can be seen that for $E_{b} / N_{0}$ values lower than $3.8 \mathrm{~dB}$, the performance improvement is little. This is because the first stage decoder is not trapped by small trapping sets at low SNR values. The length of the syndrome pattern is usually larger than the maximum length of the syndrome patterns in the look-up table, and the proposed decoder cannot correct them. At high SNR values, the FER improvement becomes apparent because more and more failures of the first stage decoder are caused by dominant trapping sets stored in our look-up table. For the same SNR value, the proposed decoder with LUT2 has better performance than the proposed decoder with LUT1. More than one order of magnitude performance improvement is achieved when $E_{b} / N_{0}=4.8 \mathrm{~dB}$ for the proposed decoder with LUT1 which contains only 1819 trapping sets, and the improvement is approaching two orders of magnitude for the proposed decoder with LUT2 which contains 16212 trapping sets. We note that the slopes of the three performance curve are different. Therefore, for even higher SNR values, the performance improvement could be more considerable. Also, it indicates that if the look-up table contains more trapping sets, the performance can be better with the compensation of memory.

\subsubsection{QC-LDPC code}

For the QC-LDPC code $\mathcal{C}_{10}, 10000$ decoding failures are collected for the quantized MS decoder. We analyze all decoding failures at $E_{b} / N_{0}=5.8 \mathrm{~dB}$ and find about $76 \%$ of them are caused by two classes of trapping sets, $(5,1)$ and $(6,1)$ classes. The total number of these trapping sets is 297. We select one member for each isomorphism sub-class and store them in the first look-up table (LUT1). The total number of trapping sets we stored is 11 . The second look-up table (LUT2) contains not only these two classes but also $(4,1),(4,2),(5,2),(6,2),(7,1),(7,2)$, and $(8,1)$ classes. The total number is 29484, but we only need to store 2097 of them. The number of trapping sets in the table is quite small which means we save the memory significantly. Actually, only $\frac{11}{297}=\frac{1}{27}$ of total trapping sets are stored in LUT1 and $\frac{2097}{29484}=\frac{1.92}{27}<\frac{2}{27}$ of total trapping sets are stored in LUT2.

Based on our previous computational complexity analysis, if we use LUT2, in the worst case, the straight-forward SPM algorithm requires 29484 array matching operations. With our proposed decoder for QC-LDPC codes, we only need $\left\lceil\log _{2}(2097)\right\rceil=12$ arithmetic comparisons to locate the matching feature. If $G_{3}^{\prime}(\mathbf{x})$ is 


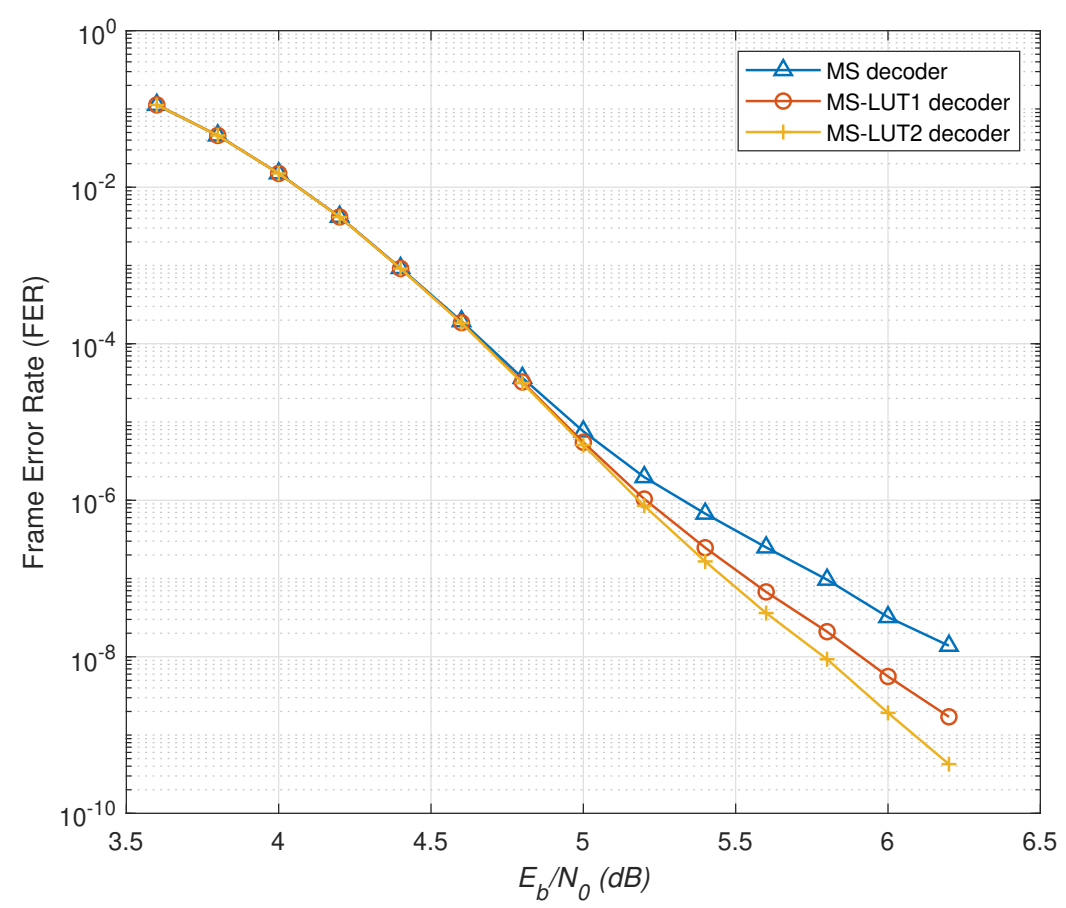

Figure 6.3: FER performances of $\mathcal{C}_{10}$ under the quantized MS decoder and the proposed look-up table based syndrome decoder with different look-up tables.

used to generate features, the average number of trapping sets that share the same feature is about 8 because the number of unique features is 263 . Then 9 more arithmetic comparisons and 8 trapping set sub-class justifications will be performed later on average. The maximum feature value is 407 . Thus we need 9 bits to represent the features. The whole feature list $\mathcal{L}_{0}$ would cost 2.36 KByte and takes $9.6 \%$ more space than only storing 2097 trapping sets which consume 24.64 KByte. Compared with storing all 29484 trapping sets, we have saved more than $92.8 \%$ memory. If we use $G_{4}^{\prime}(\mathbf{x})$ to generate features, the average number of trapping sets that share the same feature is about 5 . Then 6 more arithmetic comparisons and 5 trapping set sub-class justifications will be performed later on average. Actually, in LUT2, there are at most 23 trapping sets sharing the same feature. Then we need at most 24 arithmetic comparisons and 23 trapping set sub-class justifications. We also find there are only 8 feature values corresponding to more than 10 trapping sets, respectively. The maximum feature is 30326 . Thus we need 15 bits to represent the features. The whole feature list $\mathcal{L}_{0}$ would cost 3.93 KByte and takes $16 \%$ more space. We need less calculation but consume more memory. So we choose to use the $G_{2}^{\prime}(\mathbf{x})$ inside the 
SPM algorithm.

Fig. 6.3 shows the performances of $\mathcal{C}_{10}$ under the quantized MS decoder and the proposed look-up table based syndrome decoder with LUT1 and LUT2. It can be seen that for $E_{b} / N_{0}$ lower than $5.2 \mathrm{~dB}$, the performance improvement is little. At high SNR values, the FER improvement becomes obvious. For the same SNR, the proposed decoder with LUT2 has better performance than the proposed decoder with LUT1. About one order of magnitude performance improvement is achieved when $E_{b} / N_{0}=6.2 \mathrm{~dB}$ for the proposed decoder with LUT1, and the improvement is even larger for the proposed decoder with LUT2. We note that the slopes of the three performance curve are also different, which shows the potential for further performance improvement. Since $\mathcal{C}_{10}$ is an optimized code from standard, it also shows our proposed technique can be combined with the well-designed codes.

\subsection{Conclusion}

In this chapter, we propose a low-complexity post-processing technique to lower the error floor of LDPC codes, including an enhanced look-up table based syndrome decoder and two efficient SPM algorithms for the general LDPC codes and the QCLDPC codes, respectively. The look-up table based syndrome decoder can be used as the second stage decoder to correct the decoding failures caused by the trapping set. Compared with the similar method in literature, it is capable of handling the multiplematching problem. The proposed SPM algorithm for general LDPC codes is based on the binary search which has a complexity of $\mathcal{O}(\log N)$. It makes the syndrome pattern matching operation feasible when the look-up table stores a large number of trapping sets. The proposed SPM algorithm for QC-LDPC codes significantly reduces the number of trapping sets that need to be stored in the look-up table, while still keeps the same complexity and performance improvement ability. Both computation and storage complexities are analyzed, and a theoretically bound on the memory consumption is derived. The simulation results show that the error floors of the simulated LDPC codes are lowered. 


\section{Chapter 7}

\section{Future Works}

\subsection{Fast-Converged Check Polytope Projection Algorithm}

The iterative check polytope projection proposed in Chapter 3 avoids the complex sorting operation and achieves linear complexity. However, experiment results show that the projection algorithm may need 30 iterations to maintain the same FER performance as the accurate projection algorithm. Therefore, it is necessary to accelerate the convergence speed of the projection algorithm.

From Fig. 3.1, it can be seen that the projection coefficients $\eta_{i}$ form a geometric sequence. Therefore, the sum of these projection coefficients can be calculated by the closed-form summation formula of the geometry sequence,

$$
\frac{\eta_{1}}{1-\eta_{2} / \eta_{1}}
$$

without iterating for more than two iterations. However, for a high-dimension space, the projection coefficients do not form a geometric sequence until the projection procedure enters into a state which is equivalent to Fig. 3.1. It is possible that we stop the iteration after we find the projection coefficients $\eta_{i}$ form a geometric sequence and use (7.1) to obtain the sum of the rest of projection coefficients. It would be more interesting to study the dynamic of the projection procedure and make the projection directly enters the simple state shown in Fig. 3.1. 


\subsection{Lower the Error Floor of ADMM decoding by Post-Processing}

The post-processing technique proposed in Chapter 6 can lower the error floor of a decoder if its decoding failures in the high SNR region are mostly caused by several classes of trapping sets stored in the look-up table. So theoretically, this technique can also be applied to ADMM decoding that suffers from trapping set.

The main obstacle to apply this technique to ADMM decoding is that ADMM decoding is not stable. It means the ADMM decoder may not be trapped to a single trapping set. Instead, the ADMM decoder may be trapped into the union of several small trapping sets. Moreover, the union may not keep the same in different iterations. We can of course save the union of the small trapping sets and all the fluctuated trapping sets, but the storage consumption may be too huge. Inspired by the quantized MS decoder, a possible solution could be design a quantized version of the ADMM decoder. 


\section{List of References}

[1] R. Gallager, "Low-density parity-check codes," IRE Trans. Inf. Theory, vol. 8, no. 1, pp. 21-28, 1962.

[2] D. J. C. MacKay and R. M. Neal, "Good codes based on very sparse matrices," in IMA Int. Conf. on Cryptography and Coding, (Berlin, Heidelberg), pp. 100111, Springer, 1995.

[3] D. J. C. MacKay, "Good error-correcting codes based on very sparse matrices," IEEE Trans. Inf. Theory, vol. 45, no. 2, pp. 399-431, 1999.

[4] "IEEE standard for local and metropolitan area networks part 16: Air interface for fixed and mobile broadband wireless access systems amendment 2: Physical and medium access control layers for combined fixed and mobile operation in licensed bands," IEEE Std 802.16e-2005 and IEEE Std 802.16-2004/Cor 1-2005 (Amendment and Corrigendum to IEEE Std 802.16-2004), pp. 1-822, Feb. 2006.

[5] "ETSI standard TR 102376 v1.1.1: Digital video broadcasting (DVB) user guidelines for the second generation system for broadcasting, interactive services, news gathering and other broadband satellite applications (DVBS2)," ETSI Std. TR 102 376, pp. 1-104, Feb. 2005.

[6] "ETSI EN 302755 v1.1.1: Digital video broadcasting (DVB): frame structure channel coding and modulation for a second generation digital terrestrial television broadcasting system (DVB-T2)," ETSI EN 302 755, pp. 1-167, Sept. 2009 .

[7] "IEEE standard for information technology- local and metropolitan area networks- specific requirements- part 11: Wireless lan medium access control (mac)and physical layer (phy) specifications amendment 5: Enhancements for higher throughput," IEEE Std 802.11n-2009 (Amendment to IEEE Std 802.112007 as amended by IEEE Std 802.11k-2008, IEEE Std 802.11r-2008, IEEE Std 802.11y-2008, and IEEE Std 802.11w-2009), pp. 1-565, Oct. 2009.

[8] T. Richardson and S. Kudekar, "Design of low-density parity check codes for 5G new radio," IEEE Commun. Mag., vol. 56, pp. 28-34, Mar. 2018.

[9] "ETSI TS 138212 v15.8.0: 5G NR multiplexing and channel coding (3GPP TS 38.212 version 15.8.0 release 15)," ETSI TS 138 212, pp. 1-105, Jan. 2020. 
[10] M. P. C. Fossorier, M. Mihaljevic, and H. Imai, "Reduced complexity iterative decoding of low-density parity check codes based on belief propagation," IEEE Trans. Commun., vol. 47, no. 5, pp. 673-680, 1999.

[11] J. Chen, A. Dholakia, E. Eleftheriou, M. P. C. Fossorier, and X.-Y. Hu, "Reduced-complexity decoding of LDPC codes," IEEE Trans. Commun., vol. 53, pp. 1288-1299, Aug. 2005.

[12] J. Zhao, F. Zarkeshvari, and A. H. Banihashemi, "On implementation of min-sum algorithm and its modifications for decoding low-density parity-check (LDPC) codes," IEEE Trans. Commun., vol. 53, pp. 549-554, Apr. 2005.

[13] R. M. Tanner, "A recursive approach to low complexity codes," IEEE Trans. Inf. Theory, vol. 27, no. 5, pp. 533-547, 1981.

[14] N. Wiberg, Codes and Decoding on General Graphs. PhD thesis, Linkoping University, 1996.

[15] J. Zhang and M. P. C. Fossorier, "A modified weighted bit-flipping decoding of low-density parity-check codes," IEEE Commun. Lett., vol. 8, pp. 165-167, Mar. 2004.

[16] M. Jiang, C. Zhao, Z. Shi, and Y. Chen, "An improvement on the modified weighted bit flipping decoding algorithm for LDPC codes," IEEE Commun. Lett., vol. 9, pp. 814-816, Sept. 2005.

[17] X. Wu, C. Zhao, and X. You, "Parallel weighted bit-flipping decoding," IEEE Commun. Lett., vol. 11, pp. 671-673, Aug. 2007.

[18] G. Li and G. Feng, "Improved parallel weighted bit-flipping decoding algorithm for LDPC codes," IET Commun., vol. 3, pp. 91-99, Jan. 2009.

[19] J. Feldman, M. J. Wainwright, and D. R. Karger, "Using linear programming to decode binary linear codes," IEEE Trans. Inf. Theory, vol. 51, pp. 954-972, Mar. 2005.

[20] J. Feldman, T. Malkin, R. A. Servedio, C. Stein, and M. J. Wainwright, "LP decoding corrects a constant fraction of errors," IEEE Trans. Inf. Theory, vol. 53, pp. 82-89, Jan. 2007.

[21] R. Smarandache and P. O. Vontobel, "Pseudo-codeword analysis of tanner graphs from projective and Euclidean planes," IEEE Trans. Inf. Theory, vol. 53, pp. 2376-2393, July 2007.

[22] C. Daskalakis, A. G. Dimakis, R. M. Karp, and M. J. Wainwright, "Probabilistic analysis of linear programming decoding," IEEE Trans. Inf. Theory, vol. 54, pp. 3565-3578, Aug. 2008.

[23] N. Halabi and G. Even, "Improved bounds on the word error probability of RA(2) codes with linear-programming-based decoding," IEEE Trans. Inf. Theory, vol. 51, pp. 265-280, Jan. 2005. 
[24] M. F. Flanagan, V. Skachek, E. Byrne, and M. Greferath, "Linear-programming decoding of nonbinary linear codes," IEEE Trans. Inf. Theory, vol. 55, pp. 41344154, Sept. 2009.

[25] M. Punekar, P. O. Vontobel, and M. F. Flanagan, "Low-complexity LP decoding of nonbinary linear codes," IEEE Trans. Commun., vol. 61, pp. 3073-3085, Aug. 2013.

[26] B. Kim and H. D. Pfister, "Joint decoding of LDPC codes and finite-state channels via linear-programming," IEEE J. Sel. Topics Signal Process., vol. 5, pp. 1563-1576, Dec. 2011.

[27] N. Goela, S. B. Korada, and M. Gastpar, "On LP decoding of polar codes," in Proc. IEEE Inf. Theory Workshop (ITW), (Dublin, Ireland), pp. 1-5, Aug. 2010 .

[28] P. O. Vontobel and R. Koetter, "Towards low-complexity linear-programming decoding," in Proc. 4th Int. Symp. Turbo Codes Related Topics, (Munich, Germany), pp. 1-9, Apr. 2006.

[29] K. Yang, X. Wang, and J. Feldman, "A new linear programming approach to decoding linear block codes," IEEE Trans. Inf. Theory, vol. 54, pp. 1061-1072, Mar. 2008.

[30] M. H. Taghavi, A. Shokrollahi, and P. H. Siegel, "Efficient implementation of linear programming decoding," IEEE Trans. Inf. Theory, vol. 57, pp. 59605982, Sept. 2011.

[31] D. Burshtein, "Iterative approximate linear programming decoding of LDPC codes with linear complexity," IEEE Trans. Inf. Theory, vol. 55, pp. 4835-4859, Nov. 2009.

[32] D. Goldin and D. Burshtein, "Iterative linear programming decoding of nonbinary LDPC codes with linear complexity," IEEE Trans. Inf. Theory, vol. 59, pp. 282-300, Jan. 2013.

[33] S. Barman, X. Liu, S. C. Draper, and B. Recht, "Decomposition methods for large scale LP decoding," IEEE Trans. Inf. Theory, vol. 59, pp. 7870-7886, Dec. 2013 .

[34] S. Boyd, N. Parikh, E. Chu, B. Peleato, J. Eckstein, et al., "Distributed optimization and statistical learning via the alternating direction method of multipliers," Found. Trends Mach. learn., vol. 3, no. 1, pp. 1-122, 2011.

[35] W. Xu, X. Jiao, and J. Mu, "Evaluating the performance of turbo equalization with ADMM decoding," in 2017 IEEE Int. Conf, on Signal Process., Commun. and Computing (ICSPCC), (Xiamen, China), pp. 1-5, Oct. 2017.

[36] X. Liu and S. C. Draper, "ADMM decoding of non-binary LDPC codes in f2m," in Proc. IEEE Int. Symp. Inf. Theory (ISIT), (Honolulu, HI), pp. 2449-2453, June 2014. 
[37] X. Liu and S. C. Draper, "LP-decodable multipermutation codes," IEEE Trans. Inf. Theory, vol. 62, pp. 1631-1648, Apr. 2016.

[38] X. Jiao, J. Mu, Y.-C. He, and W. Xu, "Linear-complexity ADMM updates for decoding LDPC codes in partial response channels," IEEE Commun. Lett., vol. 23, pp. 2200-2204, Dec. 2019.

[39] X. Zhang and P. H. Siegel, "Efficient iterative LP decoding of LDPC codes with alternating direction method of multipliers," in Proc. IEEE Int. Symp. Inf. Theory (ISIT), (Istanbul, Turkey), pp. 1501-1505, July 2013.

[40] X. Zhang and P. H. Siegel, "Adaptive cut generation algorithm for improved linear programming decoding of binary linear codes," IEEE Trans. Inf. Theory, vol. 58, pp. 6581-6594, Oct. 2012.

[41] G. Zhang, R. Heusdens, and W. B. Kleijn, "Large scale LP decoding with low complexity," IEEE Commun. Lett., vol. 17, pp. 2152-2155, Nov. 2013.

[42] M. Wasson and S. C. Draper, "Hardware based projection onto the parity polytope and probability simplex," in Proc. 49th Asilomar Conf. Signals Systems and Computers, (Pacific Grove, CA), pp. 1015-1020, Nov. 2015.

[43] H. Wei, X. Jiao, and J. Mu, "Reduced-complexity linear programming decoding based on ADMM for LDPC codes," IEEE Commun. Lett., vol. 19, pp. 909-912, June 2015.

[44] X. Jiao, J. Mu, Y.-C. He, and C. Chen, "Efficient ADMM decoding of LDPC codes using lookup tables," IEEE Trans. Commun., vol. 65, pp. 1425-1437, Apr. 2017.

[45] X. Jiao, Y. C. He, and J. Mu, "Memory-reduced look-up tables for efficient ADMM decoding of LDPC codes," IEEE Signal Process. Lett., vol. 25, pp. 110114, Jan. 2018.

[46] H. Wei and A. H. Banihashemi, "An iterative check polytope projection algorithm for ADMM-based LP decoding of LDPC codes," IEEE Commun. Lett., vol. 22, pp. 29-32, Jan. 2018.

[47] Y. Lin, Q. Xia, W. He, and Q. Zhang, "A fast iterative check polytope projection algorithm for ADMM decoding of LDPC codes by bisection method," IEICE Trans. on Fundamentals of Electron., Commun. and Computer Sciences, vol. E102-A, pp. 1988-1991, Oct. 2019.

[48] Z. Liang, X. Chen, X. Sun, and L. Zhai, "A reduced-complexity ADMM based decoding algorithm for LDPC codes," in 2019 11th Int. Conf. on Wireless Commun. and Signal Process. (WCSP), (Xi'an, China), pp. 1-6, Oct. 2019.

[49] Q. Xia, Y. Lin, S. Tang, and Q. Zhang, "A fast approximate check polytope projection algorithm for ADMM decoding of LDPC codes," IEEE Commun. Lett., vol. 23, pp. 1520-1523, Sept. 2019. 
[50] F. Gensheimer, T. Dietz, S. Ruzika, K. Kraft, and N. Wehn, "A low-complexity projection algorithm for ADMM-based LP decoding," in 2018 IEEE 10th Int. Symp. on Turbo Codes and Iterative Inf. Process. (ISTC), (Hong Kong, China), pp. 1-5, Dec. 2018.

[51] I. Debbabi, B. L. Gal, N. Khouja, F. Tlili, and C. Jego, "Fast converging ADMM-penalized algorithm for LDPC decoding," IEEE Commun. Lett., vol. 20, pp. 648-651, Apr. 2016.

[52] H. Kfir and I. Kanter, "Parallel versus sequential updating for belief propagation decoding," Physica A, vol. 330, no. 1-2, pp. 259-270, 2003.

[53] D. E. Hocevar, "A reduced complexity decoder architecture via layered decoding of LDPC codes," in Proc. Signal Process. System, (Lausanne, Switzerland), pp. 107-112, Oct. 2004.

[54] J. Zhang and M. P. C. Fossorier, "Shuffled iterative decoding," IEEE Trans. Commun., vol. 53, pp. 209-213, Feb. 2005.

[55] J. Kim, M. Nam, and H. Song, "Variable-to-check residual belief propagation for LDPC codes," Electron. Lett., vol. 45, pp. 117-118, Jan. 2009.

[56] G. Han and X. Liu, "An efficient dynamic schedule for layered beliefpropagation decoding of LDPC codes," IEEE Commun. Lett., vol. 13, pp. 950952, Dec. 2009.

[57] A. I. V. Casado, M. Griot, and R. D. Wesel, "LDPC decoders with informed dynamic scheduling," IEEE Trans. Commun., vol. 58, pp. 3470-3479, Dec. 2010 .

[58] Y. Gong, X. Liu, W. Ye, and G. Han, "Effective informed dynamic scheduling for belief propagation decoding of LDPC codes," IEEE Trans. Commun., vol. 59, pp. 2683-2691, Oct. 2011.

[59] H. Lee, Y. Ueng, S. Yeh, and W. Weng, "Two informed dynamic scheduling strategies for iterative LDPC decoders," IEEE Trans. Commun., vol. 61, pp. 886-896, Mar. 2013.

[60] X. Liu, Y. Zhang, and R. Cui, "Variable-node-based dynamic scheduling strategy for belief-propagation decoding of LDPC codes," IEEE Commun. Lett., vol. 19, pp. 147-150, Feb. 2015.

[61] X. Liu, Z. Zhou, R. Cui, and E. Liu, "Informed decoding algorithms of LDPC codes based on dynamic selection strategy," IEEE Trans. Commun., vol. 64, pp. 1357-1366, Apr. 2016.

[62] J. Bai, Y. Wang, and F. C. M. Lau, "Minimum-polytope-based linear programming decoder for LDPC codes via ADMM approach," IEEE Wireless Commun. Lett., vol. 8, pp. 1032-1035, Aug. 2019.

[63] J. Bai, Y. Wang, and Q. Shi, "Efficient QP-ADMM decoder for binary LDPC codes and its performance analysis," IEEE Trans. Signal Process., vol. 68, pp. 503-518, July 2020. 
[64] I. Debbabi, N. Khouja, F. Tlili, B. L. Gal, and C. Jego, "Multicore implementation of LDPC decoders based on ADMM algorithm," in Proc. IEEE Int. Conf. Acoustics Speech and Signal Process. (ICASSP), (Shanghai, China), pp. 971975, Mar. 2016.

[65] O. Samhan and S. Draper, "Reduced message-passing bitwidth for hardware implementation of ADMM-LP decoding," in 2018 29th Biennial Symp. on Commun. (BSC), (Toronto, ON), pp. 1-5, June 2018.

[66] X. Liu and S. C. Draper, "The ADMM Penalized Decoder for LDPC Codes," IEEE Trans. Inf. Theory, vol. 62, pp. 2966-2984, June 2016.

[67] X. Jiao, H. Wei, J. Mu, and C. Chen, "Improved ADMM penalized decoder for irregular low-density parity-check codes," IEEE Commun. Lett., vol. 19, pp. 913-916, June 2015.

[68] B. Wang, J. Mu, X. Jiao, and Z. Wang, "Improved penalty functions of ADMM penalized decoder for LDPC codes," IEEE Commun. Lett., vol. 21, pp. 234-237, Feb. 2017.

[69] Y. Wei, M.-M. Zhao, M.-J. Zhao, and M. Lei, "ADMM-based decoder for binary linear codes aided by deep learning," IEEE Commun. Lett., vol. 24, pp. 10281032, May 2020.

[70] Q. Wu, F. Zhang, H. Wang, J. Lin, and Y. Liu, "Parameter-free $\ell_{p}$-box decoding of LDPC codes," IEEE Commun. Lett., vol. 22, pp. 1318-1321, July 2018.

[71] T. Richardson, "Error floors of LDPC codes," in Communication, Control, and Computing (Allerton), 41th Annual Allerton Conference on, (Monticello, IL, USA), pp. 1426-1435, Oct. 2003.

[72] M. Ivkovic, S. K. Chilappagari, and B. Vasic, "Eliminating trapping sets in low-density parity-check codes by using tanner graph covers," IEEE Trans. Inf. Theory, vol. 54, pp. 3763-3768, Aug. 2008.

[73] S.-Y. Chung, J. Forney, G. D., T. J. Richardson, and R. Urbanke, "On the design of low-density parity-check codes within 0.0045 dB of the Shannon limit," IEEE Commun. Lett., vol. 5, no. 2, pp. 58-60, 2001.

[74] X.-Y. Hu, E. Eleftheriou, and D. M. Arnold, "Regular and irregular progressive edge-growth tanner graphs," IEEE Trans. Inf. Theory, vol. 51, pp. 386-398, Jan. 2005.

[75] X. Zheng, F. C. M. Lau, and C. K. Tse, "Constructing short-length irregular LDPC codes with low error floor," IEEE Trans. Commun., vol. 58, pp. 28232834, Oct. 2010.

[76] R. Asvadi, A. H. Banihashemi, and M. Ahmadian-Attari, "Lowering the error floor of LDPC codes using cyclic liftings," IEEE Trans. Inf. Theory, vol. 57, pp. 2213-2224, Apr. 2011. 
[77] R. Asvadi, A. H. Banihashemi, and M. Ahmadian-Attari, "Design of finitelength irregular protograph codes with low error floors over the binary-input AWGN channel using cyclic liftings," IEEE Trans. Commun., vol. 60, pp. 902907, Apr. 2012.

[78] D. V. Nguyen, S. K. Chilappagari, M. W. Marcellin, and B. Vasic, "On the construction of structured LDPC codes free of small trapping sets," IEEE Trans. Inf. Theory, vol. 58, pp. 2280-2302, Apr. 2012.

[79] S. Khazraie, R. Asvadi, and A. H. Banihashemi, "A PEG construction of finite-length LDPC codes with low error floor," IEEE Commun. Lett., vol. 16, pp. 1288-1291, Aug. 2012.

[80] M. Diouf, D. Declercq, S. Ouya, and B. Vasic, "A PEG-like LDPC code design avoiding short trapping sets," in Proc. IEEE Int. Symp. Inf. Theory (ISIT), (Hong Kong), pp. 1079-1083, June 2015.

[81] X. Tao, Y. Li, Y. Liu, and Z. Hu, "On the construction of LDPC codes free of small trapping sets by controlling cycles," IEEE Commun. Lett., vol. 22, pp. 9-12, Jan. 2018.

[82] E. Cavus and B. Daneshrad, "A performance improvement and error floor avoidance technique for belief propagation decoding of LDPC codes," in Proc. 16th IEEE Int. Symp. Pers., Indoor Mobile Radio Commun., vol. 4, (Los Angeles, CA), pp. 2386-2390, Sept. 2005.

[83] Y. Han and W. E. Ryan, "Low-floor decoders for LDPC codes," IEEE Trans. Commun., vol. 57, pp. 1663-1673, June 2009.

[84] Z. Zhang, L. Dolecek, B. Nikolic, V. Anantharam, and M. J. Wainwright, "Lowering LDPC error floors by postprocessing," in Proc. IEEE Global Telecommun. Conf. (GLOBECOM), (New Orleans, LO), pp. 1-6, Nov. 2008.

[85] J. Kang, Q. Huang, S. Lin, and K. Abdel-Ghaffar, "An iterative decoding algorithm with backtracking to lower the error-floors of LDPC codes," IEEE Trans. Commun., vol. 59, pp. 64-73, Jan. 2011.

[86] X. Jiao and J. Mu, "Lowering the error floor of ADMM penalized decoder for LDPC codes," China Commun., vol. 13, pp. 127-135, Aug. 2016.

[87] X. Jiao, J. Mu, and H. Wei, "Reduced complexity node-wise scheduling of ADMM decoding for LDPC codes," IEEE Commun. Lett., vol. 21, pp. 472475, Mar. 2017.

[88] H. Wei, X. Liu, and S. C. Draper, "Correction to "The ADMM Penalized Decoder for LDPC Codes'," IEEE Trans. Inf. Theory, vol. 66, pp. 8056-8056, Dec. 2020 .

[89] H. Wei and A. H. Banihashemi, "ADMM check node penalized decoders for LDPC codes," Submitted to IEEE Trans. Commun., 2020. 
[90] H. Wei and A. H. Banihashemi, "A look-up table based syndrome decoder," In preparation, 2020.

[91] M. G. Stepanov, V. Chernyak, M. Chertkov, and B. Vasic, "Diagnosis of weaknesses in modern error correction codes: A physics approach," Phys. Rev. Lett., vol. 95, no. 22, p. 228701, 2005.

[92] C. E. Shannon, "A mathematical theory of communication," Bell System Technical Journal, vol. 27, no. 3, pp. 379-423, 1948. Bell System Technical Journal.

[93] W. Ryan and S. Lin, Channel codes: classical and modern. Cambridge University Press, 2009.

[94] S. Abu-Surra, D. DeClercq, D. Divsalar, and W. E. Ryan, "Trapping set enumerators for specific LDPC codes," in Proc. Inf. Theory Appl. Workshop (ITA), (SanDiego, CA), pp. 1-5, Jan. 2010.

[95] M. Karimi and A. H. Banihashemi, "Efficient algorithm for finding dominant trapping sets of LDPC codes," IEEE Trans. Inf. Theory, vol. 58, pp. 6942-6958, Nov. 2012.

[96] G. B. Kyung and C. C. Wang, "Finding the exhaustive list of small fully absorbing sets and designing the corresponding low error-floor decoder," IEEE Trans. Commun., vol. 60, pp. 1487-1498, June 2012.

[97] Y. Hashemi and A. H. Banihashemi, "New characterization and efficient exhaustive search algorithm for leafless elementary trapping sets of variable-regular LDPC codes," IEEE Trans. Inf. Theory, vol. 62, pp. 6713-6736, Dec. 2016.

[98] Y. Hashemi and A. H. Banihashemi, "Characterization of elementary trapping sets in irregular LDPC codes and the corresponding efficient exhaustive search algorithms," IEEE Trans. Inf. Theory, vol. 64, pp. 3411-3430, May 2018.

[99] T. Goldstein, B. O’Donoghue, S. Setzer, and R. Baraniuk, "Fast alternating direction optimization methods," SIAM Journal on Imaging Sciences, vol. 7, no. 3, pp. 1588-1623, 2014.

[100] X. Jiao, J. Mu, and J. Guo, "A comparison study of LDPC decoding using accelerated ADMM and over-relaxed ADMM," in 2016 2nd IEEE Int. Conf. on Computer and Commun. (ICCC), (Chengdu, China), pp. 191-195, Oct. 2016.

[101] B. Xia and W. E. Ryan, "On importance sampling for linear block codes," in Proc. IEEE Int. Conf. Commun. (ICC), vol. 4, pp. 2904-2908, May 2003.

[102] E. Cavus, C. L. Haymes, and B. Daneshrad, "Low BER performance estimation of LDPC codes via application of importance sampling to trapping sets," IEEE Trans. Commun., vol. 57, pp. 1886-1888, July 2009.

[103] B. K. Butler and P. H. Siegel, "Error floor approximation for LDPC codes in the AWGN channel," IEEE Trans. Inf. Theory, vol. 60, pp. 7416-7441, Dec. 2014. 
[104] M. Stepanov and M. Chertkov, "Instanton analysis of low-density parity-check codes in the error-floor regime," in Proc. IEEE Int. Symp. Inf. Theory (ISIT), (Seattle, WA), pp. 552-556, July 2006.

[105] S. K. Chilappagari, M. Chertkov, M. G. Stepanov, and B. Vasic, "Instantonbased techniques for analysis and reduction of error floors of LDPC codes," IEEE J. Sel. Areas Commun., vol. 27, pp. 855-865, Aug. 2009.

[106] M. Chertkov and M. G. Stepanov, "An efficient pseudocodeword search algorithm for linear programming decoding of LDPC codes," IEEE Trans. Inf. Theory, vol. 54, pp. 1514-1520, Apr. 2008.

[107] S. K. Chilappagari, M. Chertkov, and B. Vasic, "An efficient instanton search algorithm for LP decoding of LDPC codes over the BSC," IEEE Trans. Inf. Theory, vol. 57, pp. 4417-4426, July 2011.

[108] D. E. Knuth, The art of computer programming: sorting and searching, vol. 3. Pearson Education, 1997.

[109] Secure Hash Standard (SHS). Available at: http://csrc.nist.gov/csrc/media/publications/fips/180/4/final/documents/fips1804-draft-aug2014.pdf.

[110] W. Stallings, "Inside SHA-3," IEEE Potentials, vol. 32, pp. 26-31, Nov. 2013.

[111] D. J. C. MacKay, Encyclopedia of Sparse Graph Codes. Available at: http://www.inference.eng.cam.ac.uk/mackay/codes/data.html. 


\section{Appendix A}

\section{A New Proof of Theorem 6 of [66]}

The proof of Theorem 6 of [66] relies on its Lemma 18. However, the statement and the proof of Lemma 18 of [66] itself is not correct, leaving the proof of Theorem 6 invalid. A counterexample will be given first. Then the flaws in the proof will be pointed out. The correction to the statement and its proof will be provided later.

We use the same symbols and the definition of $\mathrm{R}_{\mathbf{c}}(a)$ here. In Lemma 18, Algorithm 1 of [66] is used to decode two channel outputs, $\mathbf{y}$ and $\mathbf{y}^{0}$, and variables within the decoding are compared on an iteration-by-iteration basis. It is said if $\mathbf{x}^{[k]}=\mathrm{R}_{\mathbf{c}}\left(\mathbf{x}^{0,[k]}\right), \mathbf{z}_{j}^{[k]}=\mathrm{R}_{\mathbf{c}}\left(\mathbf{z}_{j}^{0,[k]}\right)$ and $\boldsymbol{\lambda}_{j}^{[k]}=-\boldsymbol{\lambda}_{j}^{0,[k]}$, then $\boldsymbol{\lambda}_{j}^{[k+1]}=-\boldsymbol{\lambda}_{j}^{0,[k+1]}$. Here is the counterexample. If $\mathbf{c}=\mathbf{0}$, we obtained a trival case where the two channel outputs $\mathbf{y}$ and $\mathbf{y}^{0}$ are equal, based on the definition of $\mathbf{Z}$ in Lemma 16 of [66]. It is obvious the decoding procedure of $\mathbf{y}$ is the same as the decoding procedure of $\mathbf{y}^{0}$. Therefore, all variables during the two decoding procedures will be the same, respectively. Namely, $\mathbf{x}^{[k]}=\mathbf{x}^{0,[k]}, \mathbf{z}_{j}^{[k]}=\mathbf{z}_{j}^{0,[k]}$ and $\boldsymbol{\lambda}_{j}^{[k]}=\boldsymbol{\lambda}_{j}^{0,[k]}$ for $k \geq 0$. By initializing $\boldsymbol{\lambda}_{j}^{[0]}=\mathbf{0}$, the condition of Lemma 18 is satisfied. Based on Lemma 18, we can get $\boldsymbol{\lambda}_{j}^{[k+1]}=-\boldsymbol{\lambda}_{j}^{0,[k+1]}$ for $k \geq 0$, which contradicts the fact $\boldsymbol{\lambda}_{j}^{[k+1]}=\boldsymbol{\lambda}_{j}^{0,[k+1]}$ for $k \geq 0$.

The flaws in the proof of Lemma 18 arise first at the beginning when it says "We first verify $\mathbf{x}^{+}=\mathrm{R}_{\mathbf{c}}\left(\mathbf{x}^{0,+}\right)$. It is sufficient to verify indices $i$ where $c_{i}=1$ ". The case where $c_{i}=0$ is not proved which clearly shows that $\mathbf{x}^{+}=\mathrm{R}_{\mathbf{c}}\left(\mathbf{x}^{0,+}\right)$ does not hold. Secondly, during the proof of $\mathbf{z}_{j}^{[k+1]}=\mathrm{R}_{\mathbf{c}}\left(\mathbf{z}_{j}^{0,[k+1]}\right)$ and $\boldsymbol{\lambda}_{j}^{[k+1]}=-\boldsymbol{\lambda}_{j}^{0,[k+1]}$, the condition $c_{i}=1$ is used implicitly but the result is not verified or does not hold under the case when $c_{i}=0$. 
Before preceding, we first define an operator $T_{\mathbf{c}}(\mathbf{a})$ as

$$
\mathrm{T}_{\mathbf{c}}(\mathbf{a})_{i}= \begin{cases}a_{i}, & \text { if } c_{i}=0 \\ -a_{i}, & \text { if } c_{i}=1\end{cases}
$$

Lemma 18 can be corrected as follows.

Lemma 1. We now use Algorithm 1 to decode two channel outputs, $\mathbf{y}$ and $\mathbf{y}^{0}$, and compare variables within the decoding on an iteration-by-iteration basis. Let ${ }^{[k]}$ denote the $k$-th iteration of Algorithm 1. Consider variables in the ADMM algorithm, and denote by $\mathbf{x}^{[k]}, \mathbf{z}_{j}^{[k]}$ and $\boldsymbol{\lambda}_{j}^{[k]}$ the ADMM variables at the $k$-th iteration when decoding $\mathbf{y}$. Further, denote by $\mathbf{x}^{0,[k]}, \mathbf{z}_{j}^{0,[k]}$ and $\boldsymbol{\lambda}_{j}^{0,[k]}$ the ADMM variables at the $k$-th iteration when decoding $\mathbf{y}^{0}$. If $\mathbf{x}^{[k]}=\mathrm{R}_{\mathbf{c}}\left(\mathbf{x}^{0,[k]}\right), \mathbf{z}_{j}^{[k]}=\mathrm{R}_{\mathbf{c}}\left(\mathbf{z}_{j}^{0,[k]}\right)$ and $\boldsymbol{\lambda}_{j}^{[k]}=\mathrm{T}_{\mathbf{c}}\left(\boldsymbol{\lambda}_{j}^{0,[k]}\right)$, then $\mathbf{x}^{[k+1]}=\mathbf{R}_{\mathbf{c}}\left(\mathbf{x}^{0,[k+1]}\right), \mathbf{z}_{j}^{[k+1]}=\mathrm{R}_{\mathbf{c}}\left(\mathbf{z}_{j}^{0,[k+1]}\right)$ and $\boldsymbol{\lambda}_{j}^{[k+1] f}=\mathrm{T}_{\mathbf{c}}\left(\boldsymbol{\lambda}_{j}^{0,[k+1]}\right)$.

Proof. The iteration number is dropped for simplicity. Then $\mathbf{x}, \mathbf{z}_{j}$ and $\boldsymbol{\lambda}_{j}$ denote the variables in the $k$-th iteration, and $\mathbf{x}^{+}, \mathbf{z}_{j}^{+}$and $\boldsymbol{\lambda}_{j}^{+}$denote the variables in the $(k+1)$-th iteration when decoding $\mathbf{y}$. The similar manner is applied to variables when decoding $\mathbf{y}^{0}$. Let $\mathbf{x}=\mathrm{R}_{\mathbf{c}}\left(\mathbf{x}^{0}\right), \mathbf{z}_{j}=\mathrm{R}_{\mathbf{c}}\left(\mathbf{z}_{j}^{0}\right)$ and $\boldsymbol{\lambda}_{j}=\mathrm{T}_{\mathbf{c}}\left(\boldsymbol{\lambda}_{j}^{0}\right)$. Let $\boldsymbol{\gamma}^{0}$ be the $\log$-likelihood ratio for the received vector $\mathbf{y}^{0}$.

We first prove $\mathbf{x}^{+}=\mathbf{R}_{\mathbf{c}}\left(\mathbf{x}^{0,+}\right)$. Based on the updating rules, we know $x_{i}^{+}$is the root of

$$
x=\frac{1}{\left|\mathcal{N}_{v}(i)\right|}\left(\sum_{j}\left(\mathbf{z}_{j}^{(i)}-\frac{\boldsymbol{\lambda}_{j}^{(i)}}{\mu}\right)-\frac{1}{\mu}\left(\gamma_{i}+g^{\prime}(x)\right)\right)
$$

and, $x_{i}^{0,+}$ is the root of

$$
x=\frac{1}{\left|\mathcal{N}_{v}(i)\right|}\left(\sum_{j}\left(\mathbf{z}_{j}^{0,(i)}-\frac{\lambda_{j}^{0,(i)}}{\mu}\right)-\frac{1}{\mu}\left(\gamma_{i}^{0}+g^{\prime}(x)\right)\right)
$$

$x_{i}^{+}$is the root of (A.1) means

$$
x_{i}^{+}=\frac{1}{\left|\mathcal{N}_{v}(i)\right|}\left(\sum_{j}\left(\mathbf{z}_{j}^{(i)}-\frac{\boldsymbol{\lambda}_{j}^{(i)}}{\mu}\right)-\frac{1}{\mu}\left(\gamma_{i}+g^{\prime}\left(x_{i}^{+}\right)\right)\right)
$$

If $c_{i}=1, x_{i}^{+}=1-\mathrm{R}_{\mathbf{c}}\left(x_{i}^{+}\right), \mathbf{z}_{j}^{(i)}=1-\mathbf{z}_{j}^{0,(i)}, \boldsymbol{\lambda}_{j}^{(i)}=-\boldsymbol{\lambda}_{j}^{0,(i)}$ and $\gamma_{i}=-\gamma_{i}^{0}$. Since the 
penalty function $g$ is symmetric about 0.5 , equation (A.3) can be transformed to

$$
\mathrm{R}_{\mathbf{c}}\left(x_{i}^{+}\right)=\frac{1}{\left|\mathcal{N}_{v}(i)\right|}\left(\sum_{j}\left(\mathbf{z}_{j}^{0,(i)}-\frac{\lambda_{j}^{0,(i)}}{\mu}\right)-\frac{1}{\mu}\left(\gamma_{i}^{0}+g^{\prime}\left(\mathrm{R}_{\mathbf{c}}\left(x_{i}^{+}\right)\right)\right)\right)
$$

This means $\mathrm{R}_{\mathbf{c}}\left(x_{i}^{+}\right)$is also the root of (A.2).

If $c_{i}=0, x_{i}^{+}=\mathrm{R}_{\mathbf{c}}\left(x_{i}^{+}\right), \mathbf{z}_{j}^{(i)}=\mathbf{z}_{j}^{0,(i)}, \boldsymbol{\lambda}_{j}^{(i)}=\boldsymbol{\lambda}_{j}^{0,(i)}$ and $\gamma_{i}=\gamma_{i}^{0}$. Equation (A.3) can be transformed to

$$
\mathrm{R}_{\mathbf{c}}\left(x_{i}^{+}\right)=\frac{1}{\left|\mathcal{N}_{v}(i)\right|}\left(\sum_{j}\left(\mathbf{z}_{j}^{0,(i)}-\frac{\lambda_{j}^{0,(i)}}{\mu}\right)-\frac{1}{\mu}\left(\gamma_{i}^{0}+g^{\prime}\left(\mathrm{R}_{\mathbf{c}}\left(x_{i}^{+}\right)\right)\right)\right)
$$

It also indicates that $\mathrm{R}_{\mathbf{c}}\left(x_{i}^{+}\right)$is the root of (A.2). ${ }^{1}$ From two cases, we conclude that $\mathrm{R}_{\mathbf{c}}\left(x_{i}^{+}\right)$and $x_{i}^{0,+}$ are the roots of (A.2). Then it can be proved that $\mathrm{R}_{\mathbf{c}}\left(x_{i}^{+}\right)=x_{i}^{0,+}$ by contradiction like [66] and as a result, $\mathbf{x}^{+}=\mathrm{R}_{\mathbf{c}}\left(\mathbf{x}^{0,+}\right)$.

Then we prove $\mathbf{z}_{j}^{+}=\mathbf{R}_{\mathbf{c}}\left(\mathbf{z}_{j}^{0,+}\right)$. Let $\mathbf{v}_{j}=\mathbf{P}_{j} \mathbf{x}^{+}+\boldsymbol{\lambda}_{j} / \mu$ and $\mathbf{v}_{j}^{0}=\mathbf{P}_{j} \mathbf{x}^{0,+}+\boldsymbol{\lambda}_{j}^{0} / \mu$. For $c_{i}=0, v_{j}^{0,(i)}=\left(\mathbf{P}_{j} \mathbf{x}^{+}\right)^{(i)}+\boldsymbol{\lambda}_{j}^{(i)} / \mu=v_{j}^{(i)}$. For $c_{i}=1, v_{j}^{0,(i)}=1-\left(\mathbf{P}_{j} \mathbf{x}^{+}\right)^{(i)}-\boldsymbol{\lambda}_{j}^{(i)} / \mu=$ $1-v_{j}^{(i)}$. Then we conclude that $\mathbf{v}_{j}=\mathrm{R}_{\mathbf{c}}\left(\mathbf{v}_{j}^{0,}\right) .{ }^{2}$ By the same contradiction in [66], it can be proved that $\mathbf{z}_{j}^{+}=\mathrm{R}_{\mathbf{c}}\left(\mathbf{z}_{j}^{0,+}\right)$.

Finally, we prove $\boldsymbol{\lambda}_{j}^{+}=\mathrm{T}_{\mathbf{c}}\left(\boldsymbol{\lambda}_{j}^{0,+}\right)$ by two cases. If $c_{i}=1$, the proof of $\lambda_{j}^{+,(i)}=$ $-\lambda_{j}^{0,+,(i)}$ is given in [66]. ${ }^{3}$ If $c_{i}=0$, the proof of $\lambda_{j}^{+,(i)}=\lambda_{j}^{0,+,(i)}$ is straight-forward.

${ }^{1}$ Without the definition of operator $\mathrm{T}_{\mathbf{c}}(\mathbf{a})$ and correction about $\boldsymbol{\lambda}_{j}$, the negative sign before $\frac{\boldsymbol{\lambda}_{j}^{0,(i)}}{\mu}$ will become positive, which prevents this conclusion.

${ }^{2}$ This conclusion is not proved but used in [66].

${ }^{3}$ In [66], this part is proved by assuming $c_{i}=1$ implicitly, but it doesn't hold for $c_{i}=0$. 


\section{Appendix B}

\section{Proof of Theorem 2}

The authors in [66] proved the all-zero assumption by proving that for any channel output $\mathbf{y}$ when transmitting a non-zero codeword $\mathbf{c}$, there exists a one-to-one channel output $\mathbf{y}^{0}$ when transmitting all-zero codeword $\mathbf{0}$, such that:

- $\operatorname{Pr}(\mathbf{y} \mid \mathbf{c})=\operatorname{Pr}\left(\mathbf{y}^{0} \mid \mathbf{0}\right)$

- The decoding results (fail/success) are the same for the two cases.

Here the components of $\mathbf{y}^{0}$ are obtained by

$$
y_{i}^{0}= \begin{cases}y_{i}, & \text { if } c_{i}=0 \\ y_{i}^{\prime}, & \text { if } c_{i}=1,\end{cases}
$$

where $y_{i}^{\prime}$ is the symmetric symbol of $y_{i}$ with respect to the symmetric channel. For the AWGN channel with BPSK modulation, $y_{i}^{\prime}=-y_{i}$.

We follow the same approach in [66] to prove Theorem 2. The only differences between the ADMM CN-penalized decoder and the ADMM VN-penalized decoder are the updating rule of variable $x_{i}$ and the termination condition. The updating rule is only used to prove Lemma 18, and the termination condition is used to prove Lemma 19 in [66]. Therefore, it is sufficient to prove Theorem 2 by proving Lemma 18 and Lemma 19 in [66] hold under the new updating rule of $x_{i}$ and the new termination condition. The rest of the proof will not be repeated here.

We use the same simplified symbols and the definitions of two operators $R_{c}$ and $\mathrm{T}_{\mathbf{c}}$ with respect to a binary codeword $\mathbf{c}$ here. 


$$
\begin{aligned}
& \mathrm{R}_{\mathbf{c}}(\mathbf{a})_{i}= \begin{cases}a_{i}, & \text { if } c_{i}=0 \\
1-a_{i}, & \text { if } c_{i}=1\end{cases} \\
& \mathrm{T}_{\mathbf{c}}(\mathbf{a})_{i}= \begin{cases}a_{i}, & \text { if } c_{i}=0 \\
-a_{i}, & \text { if } c_{i}=1 .\end{cases}
\end{aligned}
$$

Lemma 2. For any codeword $\mathbf{c}$ of an LDPC code, and a function $g(\mathbf{x})$ satisfying Properties 1 to 5 defined in Sec. 5.2, equation $g\left(\mathbf{R}_{\mathbf{c}}\left(\mathbf{P}_{j} \mathbf{x}\right)\right)=g\left(\mathbf{P}_{j} \mathbf{x}\right)$ holds for any selection matrix $\mathbf{P}_{j}$.

Proof. For any codeword $\mathbf{c}$, the vector $\mathbf{P}_{j} \mathbf{c}$ contains even number of 1 s. So, there will be even number of differences between $\mathbf{R}_{\mathbf{c}}\left(\mathbf{P}_{j} \mathbf{x}\right)$ and $\mathbf{P}_{j} \mathbf{x}$. The relation between the two corresponding different components is $\mathbf{R}_{\mathbf{c}}\left(\mathbf{P}_{j} \mathbf{x}\right)_{i}=1-\left(\mathbf{P}_{j} \mathbf{x}\right)_{i}$. Based on Corollary 1, we have $g\left(\mathbf{R}_{\mathbf{c}}\left(\mathbf{P}_{j} \mathbf{x}\right)\right)=g\left(\mathbf{P}_{j} \mathbf{x}\right)$.

Lemma 3. For any codeword $\mathbf{c}$ of an LDPC code, and a function $g(\mathbf{x})$ satisfying Properties 1 to 5 defined in Sec. 5.2, equation $\frac{\partial g\left(\mathrm{R}_{\mathbf{c}}\left(\mathbf{P}_{j} \mathbf{x}\right)\right)}{\partial \mathrm{R}_{\mathbf{c}}\left(x_{i}\right)}=\mathrm{T}_{\mathbf{c}}\left(\frac{\partial g\left(\mathbf{P}_{j} \mathbf{x}\right)}{\partial x_{i}}\right)$ holds for any selection matrix $\mathbf{P}_{j}$.

Proof.

$$
\frac{\partial g\left(\mathbf{R}_{\mathbf{c}}\left(\mathbf{P}_{j} \mathbf{x}\right)\right)}{\partial \mathbf{R}_{\mathbf{c}}\left(x_{i}\right)}=\frac{\partial g\left(\mathbf{R}_{\mathbf{c}}\left(\mathbf{P}_{j} \mathbf{x}\right)\right)}{\partial x_{i}} \frac{1}{\frac{\partial \mathbf{R}_{\mathbf{c}}\left(x_{i}\right)}{\partial x_{i}}}=\frac{\partial g\left(\mathbf{P}_{j} \mathbf{x}\right)}{\partial x_{i}} \frac{1}{\frac{\partial \mathbf{R}_{\mathbf{c}}\left(x_{i}\right)}{\partial x_{i}}}
$$

For $c_{i}=0, \mathrm{R}_{\mathbf{c}}\left(x_{i}\right)=x_{i}$ and $\frac{\partial \mathrm{R}_{\mathbf{c}}\left(x_{i}\right)}{\partial x_{i}}=1$. For $c_{i}=1, \mathrm{R}_{\mathbf{c}}\left(x_{i}\right)=1-x_{i}$ and $\frac{\partial \mathrm{R}_{\mathrm{c}}\left(x_{i}\right)}{\partial x_{i}}=-1$.

Lemma 4. We now use Algorithm 4 to decode two channel outputs, $\mathbf{y}$ and $\mathbf{y}^{0}$, and compare variables during the decoding on an iteration-by-iteration basis. $\mathbf{x}, \mathbf{z}_{j}$ and $\boldsymbol{\lambda}_{j}$ are the variables in the $k$-th iteration and $\mathbf{x}^{+}, \mathbf{z}_{j}^{+}$and $\boldsymbol{\lambda}_{j}^{+}$are the variables in the $(k+1)$-th iteration of Algorithm 4 when decoding $\mathbf{y} \cdot \mathbf{x}^{0}, \mathbf{z}_{j}^{0}$ and $\boldsymbol{\lambda}_{j}^{0}$ are the variables in the $k$-th iteration and $\mathbf{x}^{0,+}, \mathbf{z}_{j}^{0,+}$ and $\boldsymbol{\lambda}_{j}^{0,+}$ are the variables in the $(k+1)$-th iteration of Algorithm 4 when decoding $\mathbf{y}^{0}$. If $\mathbf{x}=\mathbf{R}_{\mathbf{c}}\left(\mathbf{x}^{0}\right), \mathbf{z}_{j}=\mathrm{R}_{\mathbf{c}}\left(\mathbf{z}_{j}^{0}\right)$ and $\boldsymbol{\lambda}_{j}=\mathrm{T}_{c}\left(\boldsymbol{\lambda}_{j}^{0}\right)$, then $\mathbf{x}^{+}=\mathrm{R}_{\mathbf{c}}\left(\mathbf{x}^{0,+}\right), \mathbf{z}_{j}^{+}=\mathrm{R}_{\mathbf{c}}\left(\mathbf{z}_{j}^{0,+}\right)$ and $\boldsymbol{\lambda}_{j}^{+}=\mathrm{T}_{\mathbf{c}}\left(\boldsymbol{\lambda}_{j}^{0,+}\right)$.

Proof. Based on the updating rules, we have

$$
x_{i}^{+}=\frac{1}{\left|\mathcal{N}_{v}(i)\right|}\left(\sum_{j}\left(\mathbf{z}_{j(i)}-\boldsymbol{\lambda}_{j(i)}-\frac{\alpha}{\mu} \frac{\partial g\left(\mathbf{P}_{j} \mathbf{x}\right)}{\partial x_{i}}\right)-\frac{\gamma_{i}}{\mu}\right),
$$




$$
x_{i}^{0,+}=\frac{1}{\left|\mathcal{N}_{v}(i)\right|}\left(\sum_{j}\left(\mathbf{z}_{j(i)}^{0}-\boldsymbol{\lambda}_{j(i)}^{0}-\frac{\alpha}{\mu} \frac{\partial g\left(\mathbf{P}_{j} \mathbf{x}^{0}\right)}{\partial x_{i}^{0}}\right)-\frac{\gamma_{i}^{0}}{\mu}\right) .
$$

If $c_{i}=1, x_{i}^{+}=1-\mathrm{R}_{\mathbf{c}}\left(x_{i}^{+}\right)$. Combined with the knowledge $\mathbf{x}=\mathrm{R}_{\mathbf{c}}\left(\mathbf{x}^{0}\right), x_{i}=$ $\mathrm{R}_{\mathbf{c}}\left(x_{i}^{0}\right)=1-x_{i}^{0}, \mathbf{z}_{j(i)}=\mathrm{R}_{\mathbf{c}}\left(\mathbf{z}_{j(i)}^{0}\right)=1-\mathbf{z}_{j(i)}^{0}, \boldsymbol{\lambda}_{j(i)}=\mathrm{T}_{\mathbf{c}}\left(\boldsymbol{\lambda}_{j(i)}^{0}\right)=-\boldsymbol{\lambda}_{j(i)}^{0}$, and $\gamma_{i}=-\gamma_{i}^{0}$. equation (B.1) can be rewritten as

$$
\mathrm{R}_{\mathbf{c}}\left(x_{i}^{+}\right)=\frac{1}{\left|\mathcal{N}_{v}(i)\right|}\left(\sum_{j}\left(\mathbf{z}_{j(i)}^{0}-\boldsymbol{\lambda}_{j(i)}^{0}+\frac{\alpha}{\mu} \frac{\partial g\left(\mathrm{R}_{\mathbf{c}}\left(\mathbf{P}_{j} \mathbf{x}^{0}\right)\right)}{\partial \mathrm{R}_{\mathbf{c}}\left(x_{i}^{0}\right)}\right)-\frac{\gamma_{i}^{0}}{\mu}\right) .
$$

From Lemma 3, we know $\frac{\partial g\left(\mathbf{R}_{\mathbf{c}}\left(\mathbf{P}_{j} \mathbf{x}\right)\right)}{\partial \mathbf{R}_{\mathbf{c}}\left(x_{i}\right)}=-\frac{\partial g\left(\mathbf{P}_{j} \mathbf{x}\right)}{\partial x_{i}}$ if $c_{i}=1$. As a result,

$$
\mathrm{R}_{\mathbf{c}}\left(x_{i}^{+}\right)=\frac{1}{\left|\mathcal{N}_{v}(i)\right|}\left(\sum_{j}\left(\mathbf{z}_{j(i)}^{0}-\boldsymbol{\lambda}_{j(i)}^{0}-\frac{\alpha}{\mu} \frac{\partial g\left(\mathbf{P}_{j} \mathbf{x}^{0}\right)}{\partial x_{i}^{0}}\right)-\frac{\gamma_{i}^{0}}{\mu}\right),
$$

which shows $x_{i}^{0,+}=\mathrm{R}_{\mathbf{c}}\left(x_{i}^{+}\right)$.

If $c_{i}=0, x_{i}^{+}=\mathbf{R}_{\mathbf{c}}\left(x_{i}^{+}\right), \mathbf{z}_{j(i)}=\mathbf{z}_{j(i)}^{0}, \boldsymbol{\lambda}_{j(i)}=\boldsymbol{\lambda}_{j(i)}^{0}$ and $\gamma_{i}=\gamma_{i}^{0}$. We can get the same conclusion $x_{i}^{0,+}=\mathrm{R}_{\mathbf{c}}\left(x_{i}^{+}\right)$. Then, $\mathbf{x}^{+}=\mathrm{R}_{\mathbf{c}}\left(\mathbf{x}^{0,+}\right)$.

The following proof is the same as [66] because the updating rules of $\mathbf{z}_{j}$ and $\boldsymbol{\lambda}_{j}$ are the same.

Lemma 5. Let $\hat{\mathbf{c}}$ be the output of the decoder if $\mathbf{y}$ is received and $\hat{\mathbf{c}}^{0}$ be the output of the decoder if $\mathbf{y}^{0}$ is received. Then $\hat{\mathbf{c}}^{0}=\mathrm{R}_{\mathbf{c}}(\hat{\mathbf{c}})$.

Proof. The value of $\mathbf{x}$ is initialized as the hard decision value of $\mathbf{y}$ and the value of $\mathrm{x}^{0}$ is initialized as the hard decision value of $\mathbf{y}^{0}$. So

$$
\begin{gathered}
x_{i}= \begin{cases}0, & \text { if } y_{i}<0 \\
1, & \text { if } y_{i} \geq 0,\end{cases} \\
x_{i}^{0}=\left\{\begin{array}{ll}
0, & \text { if } y_{i}<0 \text { and } c_{i}=0 \\
0, & \text { if } y_{i} \geq 0 \text { and } c_{i}=1 \\
1, & \text { if } y_{i} \geq 0 \text { and } c_{i}=0 \\
1, & \text { if } y_{i}<0 \text { and } c_{i}=1
\end{array}=\mathrm{R}_{\mathbf{c}}\left(x_{i}\right) .\right.
\end{gathered}
$$

The value of $\mathbf{z}_{j}$ and $\boldsymbol{\lambda}_{j}$ are initialized such that $\mathbf{z}_{j}^{0}=\mathrm{R}_{\mathbf{c}}\left(\mathbf{z}_{j}\right)=\mathbf{0 . 5}$ and $\boldsymbol{\lambda}_{j}^{0}=\mathrm{T}_{\mathbf{c}}\left(\boldsymbol{\lambda}_{j}\right)=$ 
0. So the conditions of Lemma 4 are satisfied and then the decoding result in $(k+1)$ th iteration satisfies $\mathbf{x}^{+}=\mathrm{R}_{\mathbf{c}}\left(\mathrm{x}^{0,+}\right)$. Let $\hat{\mathbf{x}}^{+}\left(\hat{\mathbf{x}}^{0+}\right)$ be the hard-decision of $\mathbf{x}^{+}\left(\mathbf{x}^{0,+}\right)$, then it can be verified that $\hat{\mathbf{x}}^{+}=\mathbf{R}_{\mathbf{c}}\left(\hat{\mathbf{x}}^{0+}\right)$. If in $(k+1)$-th iteration, $\hat{\mathbf{x}}^{+}$can make all parity-check result equal to 0 , then $\hat{\mathbf{x}}^{0+}$ can also make all parity-check result equal to 0 . The two decoders would terminate in advance and the outputs of the decoders would be $\hat{\mathbf{c}}=\hat{\mathbf{x}}^{+}$and $\hat{\mathbf{c}}^{0}=\hat{\mathbf{x}}^{0+}$, respectively. So $\hat{\mathbf{c}}^{0}=\mathrm{R}_{\mathbf{c}}(\hat{\mathbf{c}})$. If $\hat{\mathbf{x}}^{+}$cannot make all parity-check result equal to 0 until the last iteration, the two decoders will stop after the last iteration and the outputs of the two decoders would be $\hat{\mathbf{c}}=\mathbf{x}^{+}$and $\hat{\mathbf{c}}^{0}=\mathbf{x}^{0,+}$. Again, $\hat{\mathbf{c}}^{0}=\mathrm{R}_{\mathbf{c}}(\hat{\mathbf{c}})$.

Lemma 4 and Lemma 5 act as the same role as Lemma 18 and Lemma 19 in [66]. Then Theorem 2 can be proved by the same framework. 